University of Redlands

\title{
Applying GIS to Public Gardens to Visualize and Control Irrigation Systems.
}

A Major Individual Project submitted in partial satisfaction of the requirements

for the degree of Master of Science in Geographic Information Systems

by

Ethan James Sockwell

Ruijin Ma, Ph.D.

Nader Afzalan, M.SC.

August 2015 

Applying GIS to Public Gardens to Control and Visualize Irrigation Systems.

Copyright (C) 2015

by

Ethan James Sockwell 

The report of Ethan James Sockwell is approved.
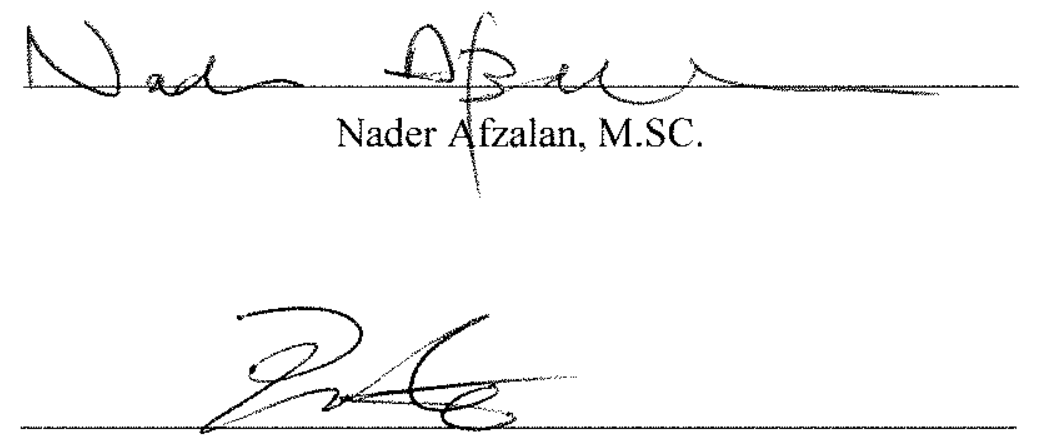

Ruijin Ma, Ph.D., Committee Chair

August 2015 



\section{Acknowledgements}

This project could not have been possible without the constant support of Chera Brown. Her support and encouragement kept me going even when facing high levels of stress, and emotional pressures. I also thank my parents for their constant support. I doubt I could have made it this far without the support and pride of my Father and Mother. To my Advisor, I thank you for pushing me through this project, your ideas and corrections, have helped grow this project to be something I can be proud of, and has helped me grow as well. 



\begin{abstract}
Applying GIS to Public Gardens to Control and Visualize Irrigation Systems

by

Ethan Sockwell
\end{abstract}

With climate changing, water shortages faced by Southern California is a serious issue. Public gardens, such as the Huntington Library, maintain a large stock of plants that need irrigation regularly, resulting in large amounts of water consumption, which often is excessive and difficult to maintain. A web based GIS was developed to manage the irrigation system at the garden spatially. This enables monitoring water usage and maintenance logs to keep the system running at peak efficiency. The project also sought to enable the collection of data by gardeners enhancing the client's ability to respond to system damage. This project resulted in three application components to view water consumption, manage data, and collect data. 



\section{Table of Contents}

Chapter 1 - Introduction ................................................................................. 1

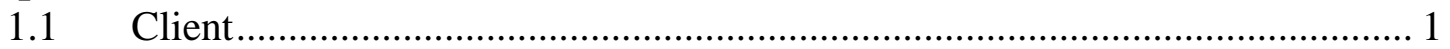

$1.2 \quad$ Problem Statement .............................................................................. 2

$1.3 \quad$ Proposed Solution ..................................................................................

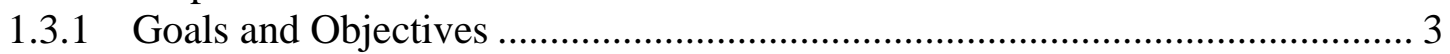

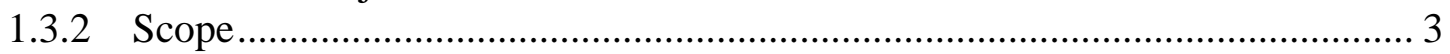

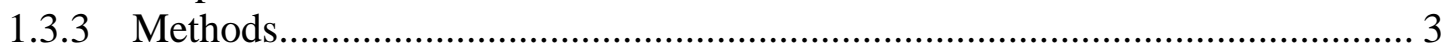

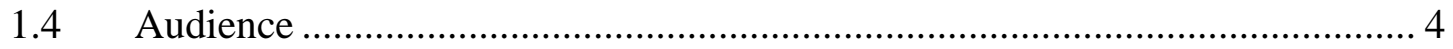

1.5 Overview of the Rest of this Report .......................................................... 4

Chapter 2 - Background and Literature Review ............................................................. 5

$2.1 \quad$ GIS and Irrigating Plants ....................................................................... 5

$2.2 \quad$ Maintenance Systems................................................................................. 7

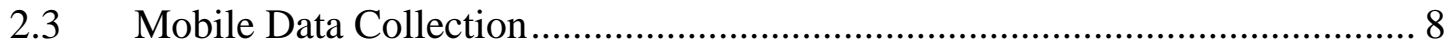

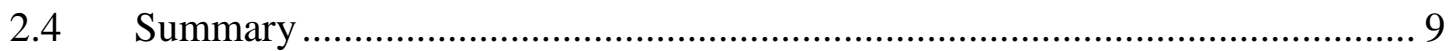

Chapter 3 - Systems Analysis and Design.................................................................. 11

3.1 Problem Statement ................................................................................ 11

3.2 Requirements Analysis ......................................................................... 11

3.3 System Design ....................................................................................... 13

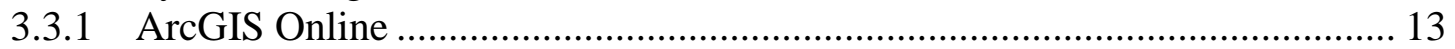

3.3.2 Mobile Collection Application ..................................................................... 14

3.3.3 Feature Management................................................................................. 14

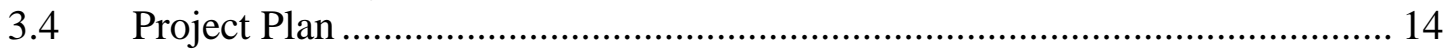

3.5 Summary ………………………………………………………….... 15

Chapter 4 - Database Design.......................................................................................... 17

$4.1 \quad$ Conceptual Data Model ....................................................................... 17

4.2 Logical Data Model .............................................................................. 18

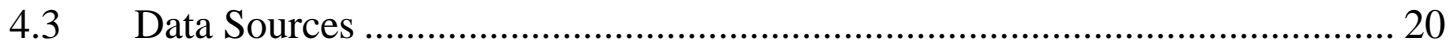

4.4 Data Scrubbing and Loading …………………….................................. 20

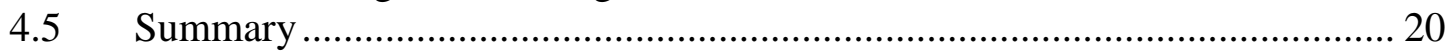

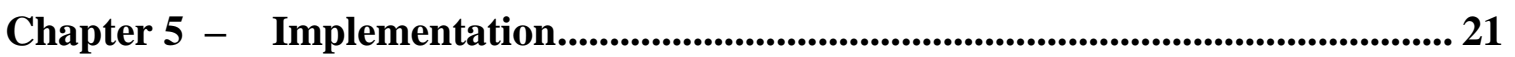

$5.1 \quad$ Publishing Feature Services.................................................................... 21

$5.2 \quad$ Creating the Web Applications .................................................................... 22

5.2.1 Authoring Web Map …………………………………………………... 22

5.2.2 Feature Editing Application..................................................................... 23

5.2.3 Water Consumption Visualization Application ................................................. 26

5.2.4 Damage Report Application ..................................................................... 30

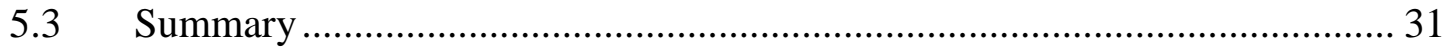

Chapter 6 - Use Cases .................................................................................................... 33

6.1 Use Case: Adding New Valves and Pipe Using Irrigation Management

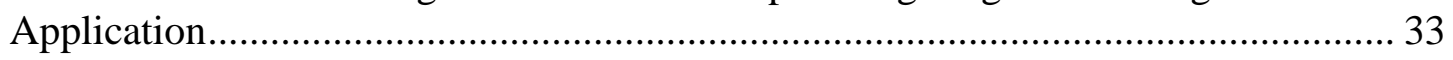

6.2 Water Consumption Application .................................................................... 37 
6.2.1 Use Case: Viewing Water Consumption for a Garden .................................. 37

6.2.2 Use Case: Valve Maintenance Logs ............................................................ 38

6.3 Use Case: Update Water Consumption Information ..................................... 40

6.4 Damage Report Application Use Case: Reporting a Leak............................. 41

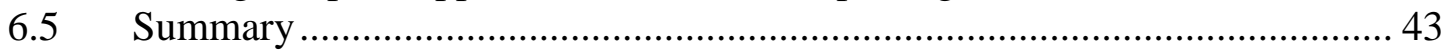

Chapter 7 - Conclusions and Future Work ............................................................... 45

Works Cited ….................................................................................................................. 47

Appendix A. Code for Viewing Application ...................................................... 49

Appendix B. Code for Report Application ................................................................... 66

Appendix C. Valve ID Assignment Script .............................................................. 70

Appendix D. Management Application File Structure.......................................... 72 


\section{Table of Figures}

Figure 1.1: Huntington Library Site Reference Map ................................................. 2

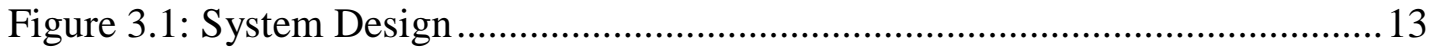

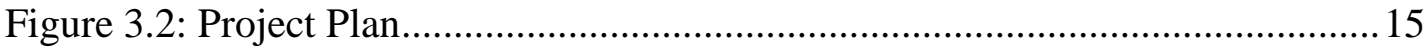

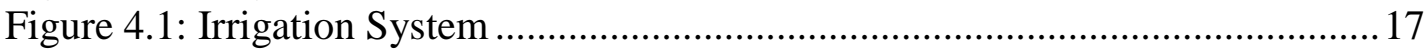

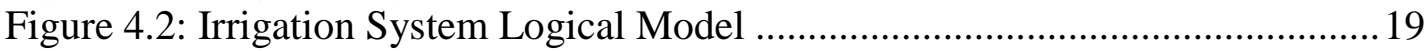

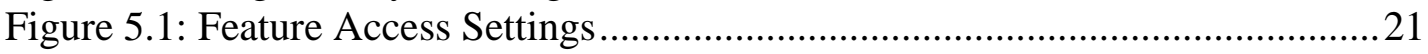

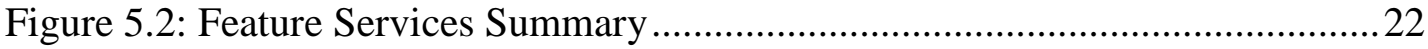

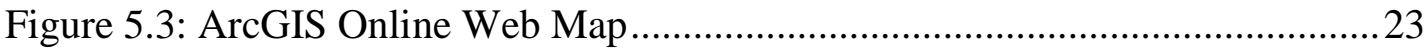

Figure 5.4: Feature Editing Application User Interface Design ..............................24

Figure 5.5: Related Records for a Selected Garden ..................................................25

Figure 5.6: Valve Search Query and Edit Box ...................................................26

Figure 5.7: Water Consumption Visualization App Design ..................................26

Figure 5.8: Layer Legend under the Legend Tab ...................................................2 27

Figure 5.9: Demonstration of Line Chart Showing Water Consumption for

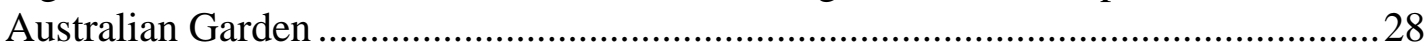

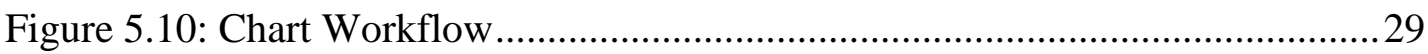

Figure 5.11: An Example of Maintenance Logs Shown in Table.............................29

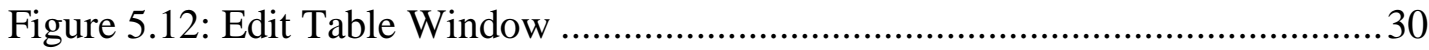

Figure 5.13: Mobile Damage Report Application ................................................. 31

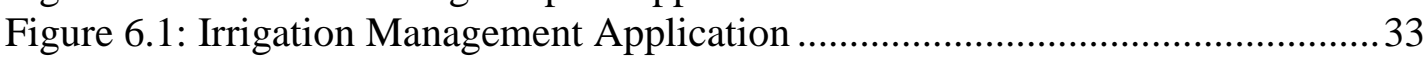

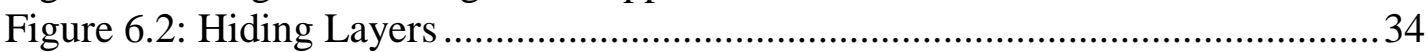

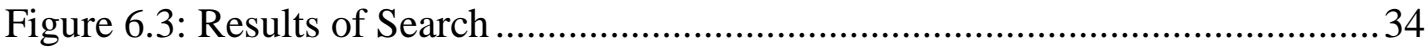

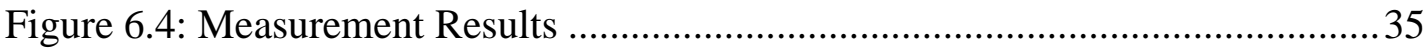

Figure 6.5: Options for Features in the Edit Window .............................................. 35

Figure 6.6: A New Pipe is Built in the Feature Layer Using the Edit Operation .......36

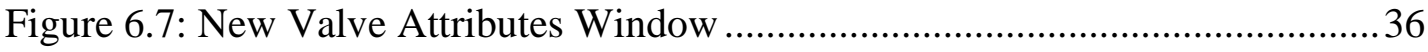

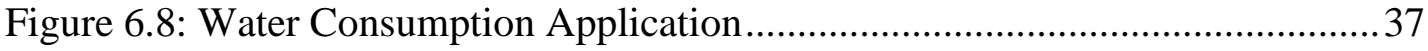

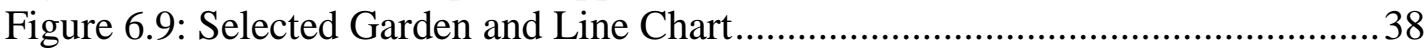

Figure 6.10: Bar Chart Showing Monthly Water Consumption ................................38

Figure 6.11: Pop-up Window Showing Valve Information.....................................39

Figure 6.12: Maintenance Table for Selected Valve ..............................................39

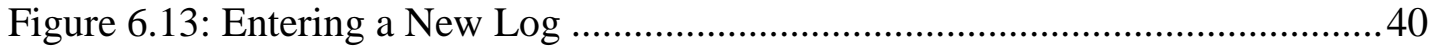

Figure 6.14: New Report Added........................................................................ 40

Figure 6.15: Searching by Garden, Month, and Year to Find Record ....................... 41

Figure 6.16: Damage Report Application ............................................................. 42

Figure 6.17: Newly Placed Point ......................................................................... 42

Figure 6.18: Date Selection Box (Left) and Filled out Values (Right).................... 43 



\section{List of Tables}

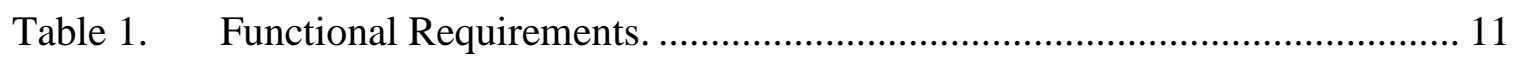

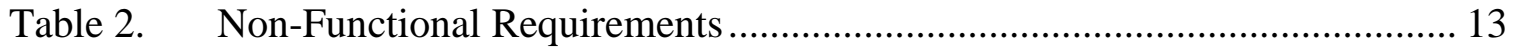





\section{List of Acronyms and Definitions}

CAD Computer Assisted Drawing

DEM Digital Elevation Model 



\section{Chapter 1 - Introduction}

California is currently in a severe drought, leading to government-mandated requirements for reduced water use. This means that organizations that use large amounts of water, such as universities and public gardens, have to take extra care to balance the use of water. This can be especially difficult for a public garden where it is required to keep the grounds green and healthy, but must also reduce water consumption. The Huntington Library, which encompasses a library, art collection, and botanical gardens, is located in San Marino, California. For an organization like the Huntington Library, it can prove very difficult to manage the beauty of their many gardens, while also keeping water use down to an economical level.

A part of the problem is that a large amount of the Huntington's irrigation information was stored in difficult-to-use drawings, and some details were only stored mentally. These mental data had no spatial linkages with existent data, and reduced the effectiveness of the data on their own. This meant that the data were not part of largescale decisions on what to repair and improve because the decision makers did not have the benefit of being able to utilize all the data available to them. Large amounts of the client's data were also out of date: valves had numbers assigned years ago using conventions no longer used on-site, and new valves did not have numbers assigned to them. While there are plans to resurvey the valves, the Huntington Library did not have anywhere efficient to place this wealth of information, so there was a good chance that it would not have been able to assist significantly in increasing water efficiency. In the previous data system, there was no reliable record of when valves underwent repairs; data lacked connection to valves on a map, due to there being no reliable numbering system, or a way to link these data. These issues made it difficult to manage the efficiency of the irrigation system, and to be aware of where the valves were currently working inefficiently.

\subsection{Client}

The client for this project was the Huntington Library, which maintains themed gardens, in addition to hosting various historical collections that are available for research and public exhibits. The point of contact was David Siversten, the Gardens Technology Coordinator. The site is located in San Marino, California as seen in Figure 1.1, and as part of its current organizational goals the Huntington Library is publicly taking action on water conservation during the severe drought that is currently occurring in California. The Huntington Library manages 150 acres of gardens, which contain more than 1,500 plant varieties, and 15 themed gardens. The client provided georeferenced aerial photos, Computer Assisted Drawing or CAD drawings of the water network, as well as expertise 
on how the groundskeepers manage the irrigation network currently within the garden.

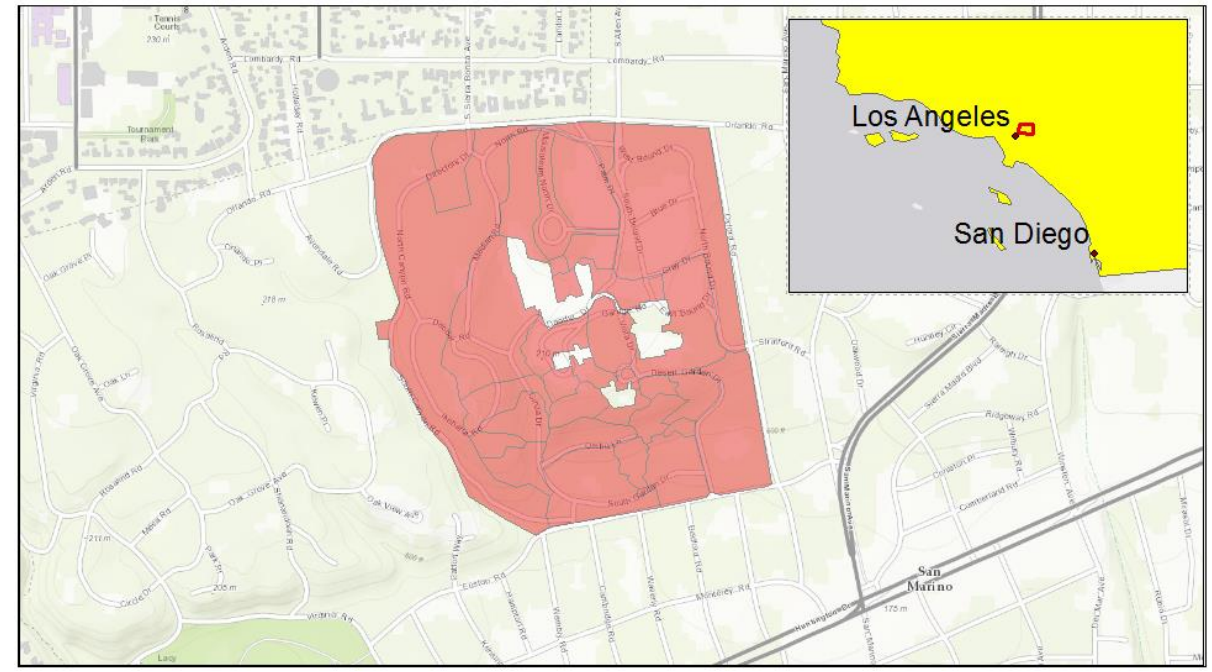

Figure 1.1: Huntington Library Site Reference Map

\subsection{Problem Statement}

The client, The Huntington Library, was unable to easily locate or collect new data on their changing pipeline network. There was a need for a method to examine the existent data on the garden's water systems to locate where the system can be more efficient, and to record notes collected by the groundkeepers. Currently a large amount of data about the irrigation system such as the valve maintenance logs, and which valves have a history of leaks have no connection to any spatial feature. Gardeners keep these leak data in mental memory, and have minimal understanding of the valve identification systems in place. They have no way to attribute this information to the valves, or to deliver this information in any meaningful way to the management. The client did not have any practical solutions for combining this mental data with the CAD Data of the pipeline configuration in place. Without addressing these problems, it would have been difficult for the client to be able to identify flaws in the irrigation system, or improve their water usage to become more sustainable with the reduced water availability.

\subsection{Proposed Solution}

The proposed solution was to build a database and a web application that will enable the user to better manage, view, and edit irrigation data at the Huntington Library. Part of this application was a light editing tool for use in the field by the less GIS experienced groundskeepers who work in the Gardens, or by other people collecting information. The datasets holds the field data, and allows the web application to function as an editing dataset. The second feature of the application was a management application for desktop use; this application allows easy editing of data attributes collected in the field, such as valve numbers and pipe types. 


\subsubsection{Goals and Objectives}

The goal of this project was to give the client a suite of applications that would allow the collection of data of various types from various sources, and compile the data into a single application for viewing and cleaning. The client could then use this master application to view all the data from these different sources in a single window. The client is then able to download the data for use in other projects or applications. The management applications were designed to operate on desktop, while the leak report application was designed for mobile use.

The objectives of this project were to provide the client with an easy better method of data collection. Since all of their data were in CAD, they needed the data moved over to an ArcGIS feature class format. Another objective was to collect data from the groundskeepers, who lack a complete understanding of the irrigation system around them in reference to their current location. They associate their information to visual representations and the actual location, rather than to a point on a map. Therefore, the application needed to allow an inexperienced user to use their current location to append a note for reference.

The main management application needed to access all the datasets, and to have layer toggles available to the user. The application also required the ability to view tables and apply edits to those tables.

\subsubsection{Scope}

Irrigation is an important issue for gardens across California. This project, however, only looked at the client's site, the Huntington Library. The project focused on irrigation pipes and valves involved in irrigation, and not the entire pipe dataset. This project looked at the value of publicly reported input on locations of leaks and other irrigation issues. In this situation, the gardeners were part of this public, though the client was able to give access to this application to any potential source of data.

This project targeted two primary audiences: the experienced members within the irrigation department at the Huntington Library, and non-GIS experienced members of the public. Due to this, there were two major components to the application, which were designed to best benefit both users. In order to keep the location of pipe outlets and important components to the system secure, the public will not have access to the pipe and valve location data.

\subsubsection{Methods}

ArcGIS Online was the primary storage for the data in this project due to being able to easily share data across the client's organization, while also allowing the use of the ArcGIS API for Javascript. All data and the relationships were configured using ArcGIS desktop, and the completed map was then uploaded to ArcGIS Online. Some minor coding was carried out in Pyscripter, using the Python language and the Arcpy module included in ArcGIS desktop. The final map contained datasets for water features, notes, and leak reports, as well as a maintenance table with a relationship configured to the valves water feature. ArcGIS JavaScript API was used to program the mobile editing tool for leaks and notes. Developer Web App Builder was configured to create the 
Management Application. Finally, an additional web application was written in JavaScript and HTML to chart the consumption of water per garden. This application also featured the ability to edit and add features to the related tables.

\subsection{Audience}

This project targets garden management individuals who are considering or in the process of applying web GIS to their irrigation management workflow. These managers and individuals with limited to advanced GIS experience will find in this project the possible solutions to problems faced by a large garden organization trying to reduce water use. There are components in this project that demonstrate how to collect crowd data from gardeners in the field, as well as how to visualize the consumption of water in the individual gardens. These methods are also relevant to garden administration who are considering GIS, and want to understand the workflows and results of implementation. Any organization with concerns about reducing water consumption or using water more efficiently such as agricultural activities, would also benefit from this project.

\subsection{Overview of the Rest of this Report}

Chapter One introduces the client, and the main elements of this project. Chapter Two examines the literature involved with the creation of this project, and elements related to this project. Chapter Three covers the system design, looks at the in-depth design elements of this project and the overall project plan. Chapter Four outlines the design of the conceptual and logical data models, how these models were applied to the data, and data sources for this project. Chapter Five covers the components used to complete this project, and the methods to their construction. Chapter Six presents the results of this project, as well as its limitations. Chapter Seven focuses on the conclusions based on this report, as well as how this project may be expanded in the future with other projects. 


\section{Chapter 2 - Background and Literature Review}

This chapter reviews literature associated with garden irrigation systems, maintenance systems, and mobile GIS to explore how to apply GIS to an irrigation system, and barrier or limitations researchers ran into that could be mitigated. Section 2.1 examines the literature on irrigation and plants. It focuses on irrigation systems for farming and garden projects, paying attention to how these researchers tackled problems and the results that they reached. Section 2.2 looks at the maintenance system to understand how to create a centralized GIS, including important components of maintenance, as well as rationales for the inclusion of maintenance within a GIS. Section 2.3 shows examples of mobile data collection to examine the methodologies used as well as risks that the projects ran into. Section 2.4 summarizes the literature review.

\subsection{GIS and Irrigating Plants}

The concept of using a GIS to solve problems that deal with irrigation is not new. At the 2005 Esri User Conference, Glissmann-Gough presented her work on the Royal Botanic Gardens, which had made the move to manage its plant collection using GPS/GIS (Glissmann-Gough, 2005). Glissman-Gough discussed how applying GIS in the gardens greatly increased the efficiency of its water usage. It is also important to note that the amount of species in the Royal Botanic Gardens is significantly larger than the Huntington Library Gardens, and they were able to get complete control of their irrigation system with less than 50 remote-controlled valves. The project demonstrates how their GIS displayed information on the pipes and valves, as well as how the Royal Botanic Gardens organized their data within a dataflow. The Royal Gardens also had issues with limited water resources, and while not dealing with a drought, they had significant issues dealing with population growth diminishing water resources. The Royal Botanic Gardens were originally consuming significant amounts of water, but thanks to the water management GIS they saved 75,000 US dollars (Glissmann-Gough, 2005, p. 3). Another important element to the solution of making water systems more efficient is the support for irrigation maintenance programs, as large amounts of water go to waste when pipes break. Glissmann-Gough also discussed how the Royal Botanic Gardens mapped tree canopy cover had an impact on the functions of their infrastructure.

Another similar example is by Durizzi, who did a project for the Desert Garden in San Diego's Balboa Park (Durizzi, 2007). Durizzi developed a GIS to help the city manage the garden. She created a basic functioning data model and workflow designed so that new GIS data could be included in the map later. Creating a basic standard is a reasonable solution to the problem of managing a large-scale garden. Her project also covered some of the issues she ran into with data, such as the points overlapping and some plant locations collected in impossible locations such as concrete paths. The system developed for the Huntington Library would need to check that the points are not in impossible locations. Durizzi also looked at the different types of analyses that are possible within a garden interface system, such as slope and aspect creation, and analysis of watering schedules and plant conditions for water conservation.

Fortes, Platnov, and Pereira (2005) explored the applications of GIS in farming irrigation to improve water use. They examined concepts about using models to simulate 
alternative irrigation schedules based on how much water the plants needed, and how much water was available. This was significant to the project because even though gardens do not use all the concepts of farms, they do share many similar features. Therefore, a model that takes into account the water available and how much stress the plants receive helps optimize water use. A major component of the model was soil moisture. Moisture sensors supplied moisture information to a GIS, which used the soil type and climate to approximate the effectiveness of the soil to hold moisture. The authors also discussed simulations using an irrigation model that takes into account various irrigation modifiers to estimate the crop yield. This model included defining an irrigation schedule and thresholds to evaluate predicted crop yield and water use of the irrigation schedule (Fortes, Platnov, \& Pereira, 2005). This was useful because it showed the value of using minimum irrigation requirements in the solution. These data could then be compared to current levels to increase or decrease water levels.

Liu (2009) discussed the use of GIS and models in large-scale crop management. Part of these models worked with GIS to take into account site-specific inputs such as climate, soil, and land use to give estimates of crop yield. This crop yield output combined with a GIS to give a graphic representation of the irrigation model. The factors used were climate, soil, land use, irrigation, and a DEM. While there may not be much use for the land use factor at the Huntington Library since it is private property, utilizing soil and climate data may be a key part of modeling the effectiveness of a predictive model. These layers combine to form a prediction of soil moisture and retention, as keeping water in the soil and near the roots is the goal of any agricultural system. DEMs would also be useful as they can be used in water flow analysis, unless the Gardens are utilizing a direct drip method. Since the Huntington Library has large lake features, having flow analysis may help predict water levels for those lakes (Liu, 2009).

Clark's (1998) discussion on hydrology brought up concerns about the resolution of such data to be used. Since higher resolutions increase data volume, it causes problems in storage and speed. While technology has improved since the time of the article, it did bring up concerns of how detailed a solution would need to be for a garden. If the data were clipped to the extent of the garden, it reduces data size, and allows the application to perform faster. An application could still negatively affect draw time and interaction speeds on a mobile solution if it is too bulky. Clark (1998) also said that having high resolution did not necessarily mean clearer solutions, as features in GIS were unclear and non-specific, and that increased resolution does not improve the use of the solution.

Satti and Jacobs (2004) examined using GIS to analyze the overall regional drought water demand. Some of the components within their research applied to the separate regions within Huntington gardens, such as modeling demand for water in each individual garden. They stated that when constructing a GIS to predict irrigation water demands the important elements were soil type and the irrigation system features. Satti and Jacob's GIS looked at the watering practices used in fields in order to explain water usage and needs for those areas. At the Huntington, it is important to understand the attributes needed to identify the watering methods used in each garden or irrigation region to facilitate water reduction. This would make it easier to analyze or predict the actual water needs, and the consumed water for a given area. Satti and Jacobs (2004) also used an object-focused database in order to manage its information and mentioned the struggles with GIS to maintain temporal data. This brings up the value of allowing 
shifting changes to the system, showing that information such as past and future pipe installation dates is important. They reviewed methods that are applied to farms, expanding them to predict the demands for a region. These methods apply to the Huntington Library, and their multiple garden regions.

\subsection{Maintenance Systems}

The next major component considered for this project was the applications of GIS to irrigation systems maintenance. One of the initial topics was the use of GIS to predict and measure the quality of elements within a system. Serre, Peyras, Tourment, and Diab (2008) used GIS to model and access levees in Europe and France to determine locations where breakages could occur. This allowed the replacement of defective levees before a flood incident occurs. One of the important elements of the process was the usage of a qualitative scale based on indicators and preset criteria. Within this example, Serre et al. (2008) configured criteria such as tree roots near the levee that have a severity rating. For this criteria they used a 10 point scoring method to show the risk value to the nearby levees based on that criteria, which then assigned the highest possible value to the levee. Their method generated a line that was broken up and symbolized based on the risk that each of the levee features faces. Applying this to a garden irrigation system, it could check for certain criteria, such as leak reports, or the number of instances of maintenance in the last six months to create a risk number from these variables, assigned to the valves. The Huntington Library can use this number to prioritize maintenance before a major breakage of a pipe or valve occurs within the garden.

Tabesh and Saber (2012) discussed prioritization for rehabilitating water networks, also utilizing a risk number system. They looked at the value of nodal water pressure, or how each element of the system faced strain separately. Each pipe or valve had a unique strain within the system, which could be different from even nearby units. This demonstrates the value of having information on each element of the system allowing an efficiency assessment of the system as a whole. It is possible for a system to flag valves that are under heavy strain due to either lack of maintenance or other issues within the system. Tabesh and Saber(2012) argued that having a system that looks at each individual asset within a system and measures their risk states valuable to long-term management of systems, especially when dealing with water.

Halfawy, Pyzoha and El-Hosseiny (2002) investigated the value of an integrated infrastructure data model. Within their model, they included inventory and condition data, performance data, maintenance planning, and deterioration. Most of the components of this integrated infrastructure model focused on how often elements of the system were repaired or damaged, and whether or not those elements needed to be replaced. It also included the financial cost data which, when brought in after all of the other elements were considered, allowed the cost-to-benefit ratio of replacing an element of the system to be considered. Booth and Rogers (2001) also brought up the idea of integration of information for improved assessment potential in an article where they covered the value of using GIS to manage infrastructure capital assets. They covered the value of an asset inventory where "utility managers inventory their assets and make decisions regarding maintenance, preservation of value and function, and replacement" (Booth \& Rogers, 2001, p. 68). This was applied to public gardens, since managing an irrigation system on a large scale, with several gardens managed by different individuals, is the same as 
managing a large civic operation with multiple utility managers and components. Thus, a unified and centralized data manager is a useful design-making resource and, as Booth covered, a cost-effective solution.

Observing wider topics involving centralized GIS data storage brings up the possibilities of using related tables to store large amounts of data attributed to a singular feature. Gogu et al. (2001) covered this in an article where they created a hydrogeological database powered by GIS. They covered the methods used in assembling their database, and their use of related tables to store information for assets. For example, the Sample ID may link a single sample to a table of parameters. Maintenance logs for valves at the Huntington Library can utilize this related table approach. If valves had unique IDs that linked the valves to a listing of all instances of its maintenance, this could be a data source for the irrigation system. Gogu et al. (2001) further support this when they looked at individual stations, each of which was linked to a table of water levels recorded by that station. This one-to-many relationship can be applied to valves at the Huntington Library as well. The water levels as recorded by the individual valves could be recorded in a related table, so that each water level record links to a valve and each valve links to all of its records. Expanding further on their ideas, Gogu et al. (2001) captured monthly volumes of water based on recorded flow data. This allowed them to report the monthly consumption of water. At the Huntington Library, the monthly consumption of water for a large area such as individual gardens would be captured, allowing users to better compare water consumption between gardens.

\subsection{Mobile Data Collection}

Lwin and Murayama (2011) set up and utilized a Post Office Protocol (POP) mail server to help facilitate field data collection using only mobile phones. While this may not be as convenient as the present-day Esri ArcGIS Collector, it offered a unique approach and third-party method to collect field data to be inserted into a feature class. It worked by sending the data collected by the mobile device that has a GPS and sending an email to a POP3 server (Lwin \& Murayama, 2011). This server then took the text and translated it into a Microsoft Access database, which was connected to a GIS server. The GIS was where the data were converted into an Esri shapefile. They mentioned the need to clean up data that was collected via mobile, since users were prone to mistyping information or clicking in the wrong location. For the Huntington Library it may be helpful to consider using domains to simulate their solution of using code designations. Simplifying the collected data or using dropdown menus rather than text boxes decreases the chance of individuals entering errors into the system.

A blog by Jeff Shaner (2015) covered the value of related tables in infrastructure, such as those used to record maintenance logs for fire hydrants. Shaner covered the new features of the ArcGIS collector to add and modify related tables, a new field data collection method supported by Esri Collector. It covered how data were configured to allow the collector to modify and add to related tables, along with examples of related tables used in several domains. This improvement allowed individuals with a smartphone and an organizational ArcGIS Online account to easily modify and add data to related tables by simply selecting the feature on a hosted webmap. This feature also maintained domains associated with the table, allowing a user to either select from a dropdown menu or use the current date for a date field. 
Mobile GIS has significant value in managing real-time field data, as well as issues of environmental concern. Tsou (2004) explored using a mobile GIS platform to collect real-time mapping data when monitoring habitats. He also pointed out that the main users of a mobile GIS were field workers deployed in an area of interest. He covered the principles of mobile GIS in creating a method for a mobile phone device to request information from a server and then update that information (Tsou, 2004). He also discussed several challenges to implement wireless mobile GIS, the first of which was the short range of wireless networks. The second challenge he raised was that the screen displays of the mobile devices were small, which made it difficult to manipulate the layers on the screen. Users also had issues viewing the screen in sunlight. For the Huntington library project, it will be important to recognize the need to keep the user interface small, so that it can be used effectively despite being on a small mobile device. Tsou(2004) also discussed the issue of data security when using mobile GIS applications. The security of client information when going mobile is still a relevant issue today.

Reichenbacher (2001) covered Location Based Services (LBS), which were services that adjust based on your current location. An example given of how this LBS was applied to situations was the opacity of points being adjusted on the fly, due to a user's current location or objectives. Based on what users were doing, it would show them relevant information on their mobile GIS. Within a garden setting, a system would notice if you are moving into the proximity of a certain type of plant repeatedly or searching for the same family of plant, and highlight those plants for you automatically.

In the case of irrigation system management, it could highlight nearby pipes that have been creating trouble. An important element that Reichenbacher (2001) discussed was creating applications that looked at context, or what your user is trying to do, and design the application to adjust visually to serve that purpose. He discussed several variables to take into account when creating the context for a mobile application: who the user is, where they are, what time it is, and what the user is doing are all valuable factors for a developer to consider and for an application to adjust based on. Other examples of these variables were Location, Time, and Activity (Reichenbacher, 2001).

\subsection{Summary}

The literature reveals that assembling a centralized data system is the ideal solution to the problem that the Huntington Library faces. Allowing the users to easily view and access the state of individual components within the system and view the strains on individual pipes, allows those users to better access whether individual elements of the system need to be replaced to increase efficiency, as well as to better measure the cost-effectiveness. The literature also points out the value of utilizing related tables in order to capture data such as maintenance tables, leak reports, or even overall water consumption. Including analysis that allows users to easily view which pipes are at risk and compare the consumption of the gardens will also allow easy prioritization of water reduction attempts while still taking into account the location of plants and the individual qualities of each garden, such as prominent soil types.

This project needed a management application to allow the Huntington Library to create new features within the dataset easily, and create a single unified source to view their data. As shown in the literature, having centralized data greatly increases efficiency and costs when managing any complex system, and having access to all the variables in 
the field increases the ability to manage these systems. Maintenance systems tables were needed because the related tables were the best way to allow multiple records to be assigned to the individual valves, making these records spatial and easily viewed when compared to other spatial data within the system. However, just seeing the tables was not enough, in order to better adapt to changes and visually see the impact on variables such as consumption it was necessary to implement an application to visualize and work with these related tables.

The literature also supported the value of including mobile access to the data, and the capture of information from individuals in the field. This meant that a simple application that users could use without needing to navigate complicated menus. Finally, this literature shows the need for all these application components to work in unison on the same dataset, so the data were hosted on ArcGIS Online, allowing all the applications to edit these features, and see the edits from other applications. 


\section{Chapter 3 - Systems Analysis and Design}

This chapter covers the overall system design, and the requirements decided for the project based on the Problem Statement in section 3.1. In Section 3.2 the requirements for the project are analyzed and listed. These requirements are the means with which this projects success is measured. Section 3.3 looks at the system design, and how the various pieces of this project worked together to fulfill the client's needs. Section 3.4 looks at the overall plan for the project, and the steps to be taken to complete this project. Section 3.5 summarizes this chapter.

\subsection{Problem Statement}

The client, The Huntington Library, had difficulty in connecting their data to their irrigation system. Maintenance logs lacked a connection with the valve, which meant that the logs did not know where the valve they had serviced was, what type of valve it was, or even the type of water the valve serviced. There was no way for the client to filter the maintenance logs by a single valve. Large amounts of data were stored in CAD diagrams, or merely kept in the memory of individuals. This made it difficult for the client to make decisions as to which valves need special attention from management, or even be replaced in the long term. Creating a system to manage all these data and view them spatially would allow the Huntington to better manage their water usage, and insure the delivery of water to their gardens as needed.

\subsection{Requirements Analysis}

These requirements were generated by discussions with the client in order to insure that the solution met the client's needs. This step was taken prior to any, to insure that the project best accomplished the goals and needs of the client. There are two types of requirements for a project: functional and non-functional. The functional requirements identify the operations that the program must complete on a user command or interaction. Non-functional requirements are the requirements not fulfilled by a single click or operation, but can still be used to judge the effectiveness of the solution.

Table 1. Functional Requirements.

\begin{tabular}{|l|l|}
\hline Requirement & Description \\
\hline Toggle Layer Visibility & $\begin{array}{l}\text { The application must be able to } \\
\text { toggle the layers contained within it } \\
\text { so that the user can view only what } \\
\text { is necessary for the current job. This } \\
\text { prevents the user from being } \\
\text { overwhelmed by all the data } \\
\text { available, but also be able to bring in }\end{array}$ \\
\hline
\end{tabular}




\begin{tabular}{|c|c|}
\hline & $\begin{array}{l}\text { and manage all data in a single } \\
\text { application. }\end{array}$ \\
\hline Print Download Maps & $\begin{array}{l}\text { This function allows the user to } \\
\text { download the current view into a } \\
\text { PDF so that it can be shared with } \\
\text { other members of the department } \\
\text { easily, or so that it can be used in } \\
\text { presentations in financial meetings } \\
\text { or in conversation with higher } \\
\text { management. }\end{array}$ \\
\hline Edit Feature Layers & $\begin{array}{l}\text { Since large amounts of the data will } \\
\text { be collected via the custom app } \\
\text { collector or Esri's off the shelf } \\
\text { collector, there is a possibility for } \\
\text { data to be entered incorrectly. It is } \\
\text { also possible for the client to use an } \\
\text { alternate workflow of filling out the } \\
\text { minimal amount of information into } \\
\text { the collector device, and fill in the } \\
\text { rest when in the office. }\end{array}$ \\
\hline Measure Distances & $\begin{array}{l}\text { Allow the user to measure distance } \\
\text { between features. }\end{array}$ \\
\hline Select Features Based on Attributes & $\begin{array}{l}\text { A Widget that allows the user to } \\
\text { select a valve based on its ID. This } \\
\text { will allow the client to quickly select } \\
\text { and search for the valves. }\end{array}$ \\
\hline Review Features Information With Pop-ups & $\begin{array}{l}\text { The client is able to select a feature } \\
\text { on the map and view a pop-up with } \\
\text { information related to that point. In } \\
\text { the case of valves, the client will } \\
\text { also be able to view the } \\
\text { Maintenance tables associated with } \\
\text { that valve. }\end{array}$ \\
\hline
\end{tabular}

The functional requirements demonstrate the actions that are processed by the program via clicks or user interaction. These functions are what the program actually 
does as user-controlled functions. These are the mandatory limits to what the program must be able to accomplish and do. These requirements take a user interaction and generate an output. Table 2 outlines the non-functional system requirements below.

Table 2. Non-Functional System Requirements

\section{Requirement}

Graphic User Interface for Navigating

through the application

Web Server to Host Applications

\section{Description}

The applications should be easy to use, especially the custom note collector, since it will be used by people with little to no experience with GIS.

A server will be required to host the Web Applications from this project.

\subsection{System Design}

The system has three major components: the data stored on ArcGIS Online, the collector applications that interact and modify that data in the field, and the WebApp Management Application (Figure 3.1).

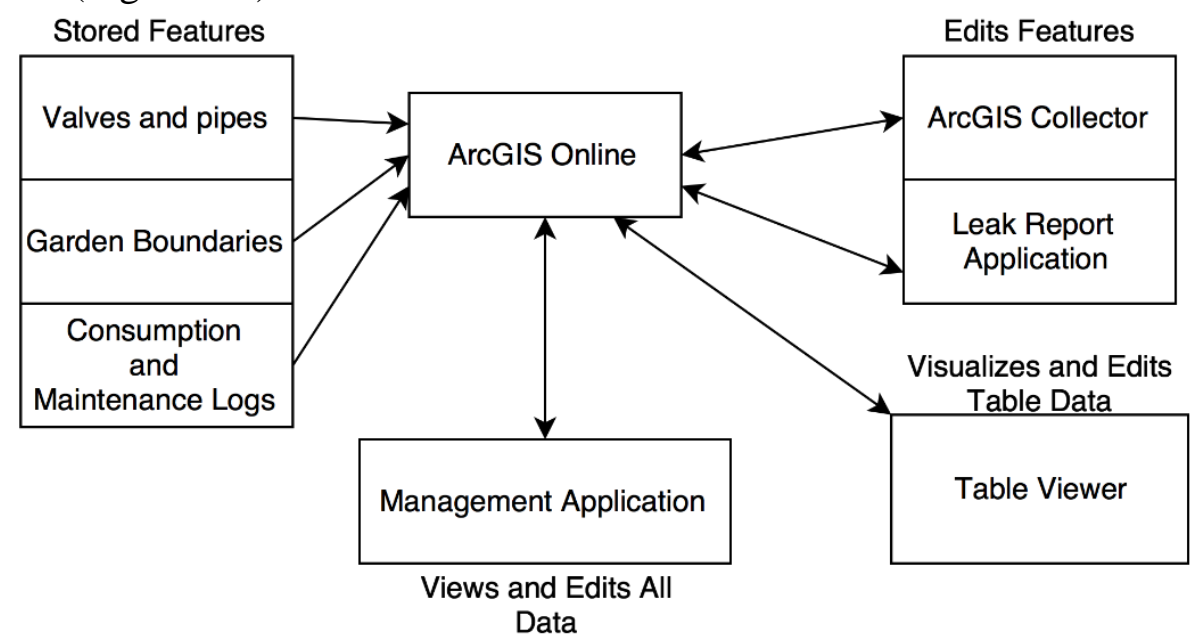

\section{Figure 3.1: System Design}

\subsubsection{ArcGIS Online}

The system used ArcGIS Online to host the data, as the client had limited server resources. ArcGIS Online was also ideal to fulfill the requirement to edit data in the field. This combination of requirements made it necessary to focus on utilizing the organizational ArcGIS Online for data storage, rather than a server-stored geodatabase. The inclusion of related tables in ArcGIS Online allows the maintenance information to be managed by a stand-alone table. This table can then be edited by using ArcGIS Collector and JavaScript Applications. 


\subsubsection{Mobile Collection Application}

A custom application written using JavaScript allowed for the collection of visually confirmed issues while in the field, which could be shared as a public data repository. This allows the application to be shared with the Gardeners or others who may be working on-site, without having to give the individual full organizational account access. This component of the application stores the identified issue, date and time, and location. The purpose of this branch of the system was to collect the visual knowledge of the gardeners that may be lost due to their difficulties in recalling where they were when they observed the damage.

The layers on ArcGIS Online are also viewed by the table viewer application. This application is written in JavaScript, HTML, and CSS. It maintains the relationships between features and allows visual representation of the data within the related tables.

\subsubsection{Feature Management}

The other component of the application focuses on managing the Irrigation features. This component captures information such as model number, type, size, location, and a unique identifier. The app assigns a unique ID to the valves, as the client currently had no operating identifier schema for the valves. While older valves had a number ID associated with them, newer valves had no values in their system, and so they would accidentally inherit nearby valve values, which would make a relationship of one to many impossible. This system will use a unique ID to link the valve to the maintenance logs, which will reference this new valve ID system.

This web map is accessed via a management application written in JavaScript and HTML, using the Esri Developer Web App Builder as a thematic base. It connects and enables a user to edit features, and is accessed via a web browser.

\subsection{Project Plan}

There were several phases to the project, the initial phases of communication and problem identification, the design phase, development, testing, quality control, deployment, and closing. As elements of the project were completed, testing was initiated to insure that it met the quality control standards for that element. If it failed, it would then be returned into the design or development stage to bring it up to the needed standard. An overview of this workflow and project phases is shown in Figure 3-2. 


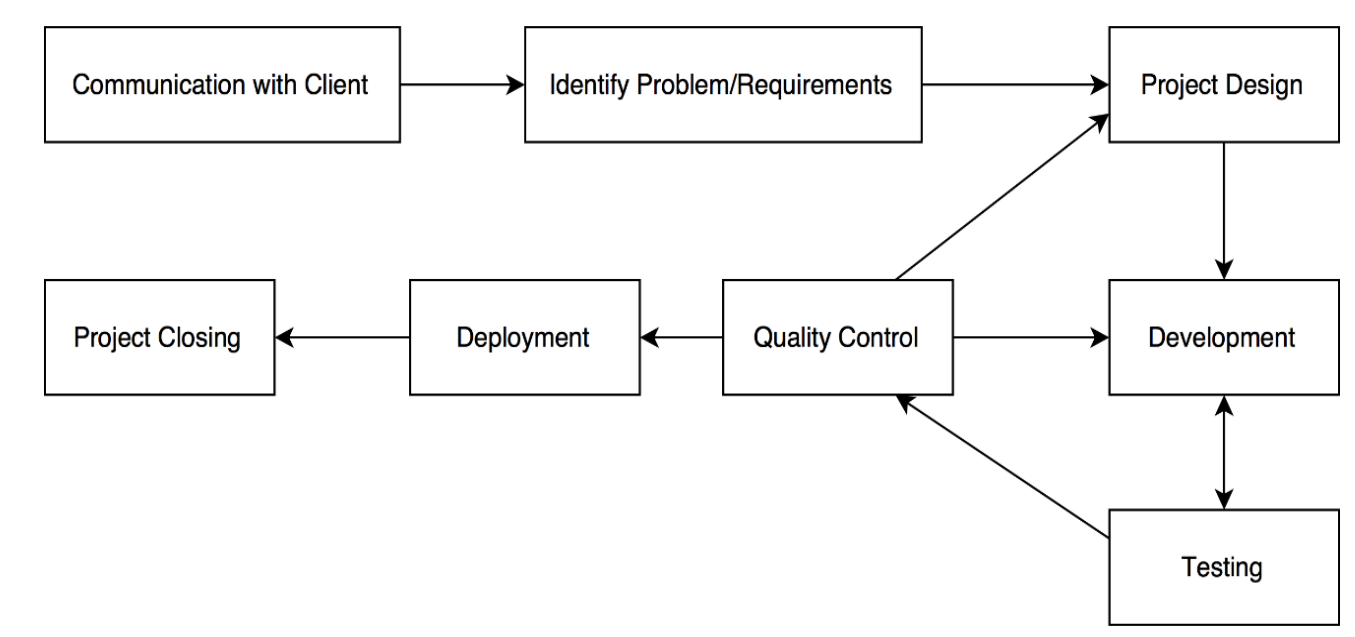

Figure 3.2: Project Plan

During the communication phase conversation with the client sought to access the problems within current solutions and workflows. It was after this communication that the identification phase was reached. During this phase, communication with the client identified the requirements based on infrastructure, and the needed solutions to the problems identified by this project. It was also during this phase that the problem was defined and stated.

During the initial project design phase, the client provided the initial data in the form of CAD data and aerial photography. These data were converted into ArcGIS ready feature classes, and new feature attributes were added. It was at this point that the overall system was also designed, and major design choices were made.

In the next phase a Python Script was used to assign a unique primary key to the features, so that they could be linked with a maintenance table. These data were then uploaded to the Huntington ArcGIS Online organizational account. Next, the web application was designed and configured. During this phase, the JavaScript note collector app, and table viewing app codes were also written.

After the initial work was completed, various tests were designed, such as testing the collector applications in various web browsers, and on the client's mobile devices. Next, the webapp was tested on the desktop using the client's favored browser, and various other popular web browsers as well. This workflow was designed to insure quality for project components and that if components failed they would be returned to development for improvement.

The applications were then delivered and installed on the client's systems in the implementation phase. A day of training was provided to instruct the client on the use of the program. Materials were also provided to list and define the elements of the application and what could be repaired, as well as tips for expanding the program.

\subsection{Summary}

This chapter covered the problem, system requirements, system design, and project plan. The project requirements are stated as were agreed upon by the client, and lists functional requirements as well as non-functional requirements. The project itself had 7 phases that produced several products. This project also featured a cycle for quality control, to insure 
that the product met the standards defined and agreed upon with the client. This chapter also explained tasks associated with each of these phases, and the overall flow of work for completing this project. 


\section{Chapter 4 - Database Design}

This project used several datasets to illustrate the interactions within the Huntington Gardens irrigation system, and the various elements required to maintain them. These models also take into account the flow of water and the needs of the plants within the gardens. These models provide the framework for how the data were managed to accomplish project goals. In this chapter, Section 4.1 examines the conceptual model for the project. This model shows the relationships presented by the client, and the on-site issues. A logical model was constructed for this project to illustrate the choices made in the data configuration to illustrate the conceptual model. This logical model can be viewed along with its discussion in Section 4.2. The sources for the data are discussed in Section 4.3, and the process involved in preparing and loading the data for use is covered in Section 4.4. This chapter concludes with a summary in Section 4.5.

\subsection{Conceptual Data Model}

This conceptual model represents the interactions between the elements of the irrigation system at the Huntington Library. This model followed the flow of water through pipes and valves. Also included in this model were the involvement of maintenance individuals that maintain these pipes and valves, as well as factors involved in the flow of water. The conceptual model is shown in Figure 4.1.

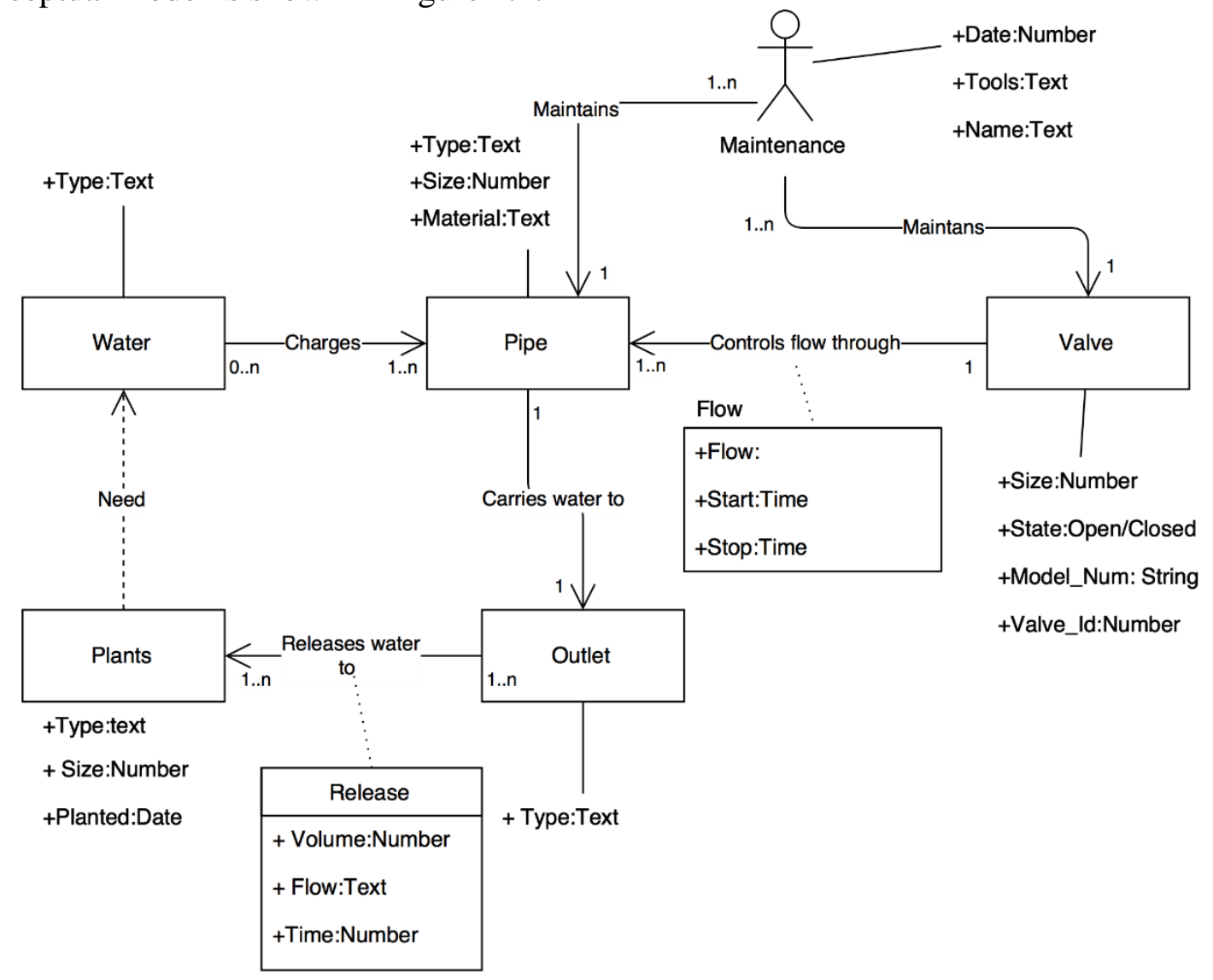

Figure 4.1: Irrigation System 
The irrigation system's objective is to deliver water to plants that require it. The method the system follows to accomplish this is demonstrated by the model, illustrated in Figure 4.1 While there are drought tolerant plants and some plants which do not need water, the assumption for this model was that any plant included in an irrigation system requires water. The model started with the flow of water into the system, which charges the pipes entering the primary structure. The water used to charge the system has several different types: potable, non-potable, irrigation, fire. The type of water in the system determines what it can be used for, since some water is recycled. Most water within the irrigation system was simply marked as the irrigation type. Another important interaction in this model was that valves control the flow of water through pipes. These valves are the major nodes where the client has the most control over the flow of water. These points are also where the client is most easily able to measure the flow of water through the system, which is why there was a flow variable for start and end time, as well as flow amount. Outlets connect to the pipe system, and are where water dispenses to the plants by gardeners. In this system, valves are the outlet points that the client has the most control over, whereas outlets are merely the ends of pipes that release water outside of the system. The maintenance department logs their repair operations and manages the crew that maintains these pipes and valves. These repair actions take place on a date, with certain tools, and by a certain maintenance individual. This view of the system was used to construct the logical data model in the following chapter.

\subsection{Logical Data Model}

The logical data model looked at how the data were organized based on the conceptual model. This model also showed the tables and connections utilized to best illustrate the conceptual model above spatially. Figure 4.2 demonstrates the logical model for this project. It shows the Irrigation features which contain valves and pipes, and these valves are linked to the maintenance logs table. The field data feature contains the leak and note reports. Garden boundaries contain the polygons for all the gardens, and is joined to the water consumption table based on the GardenID. All of these features were stored on ArcGIS Online as the irrigation system webmap. 


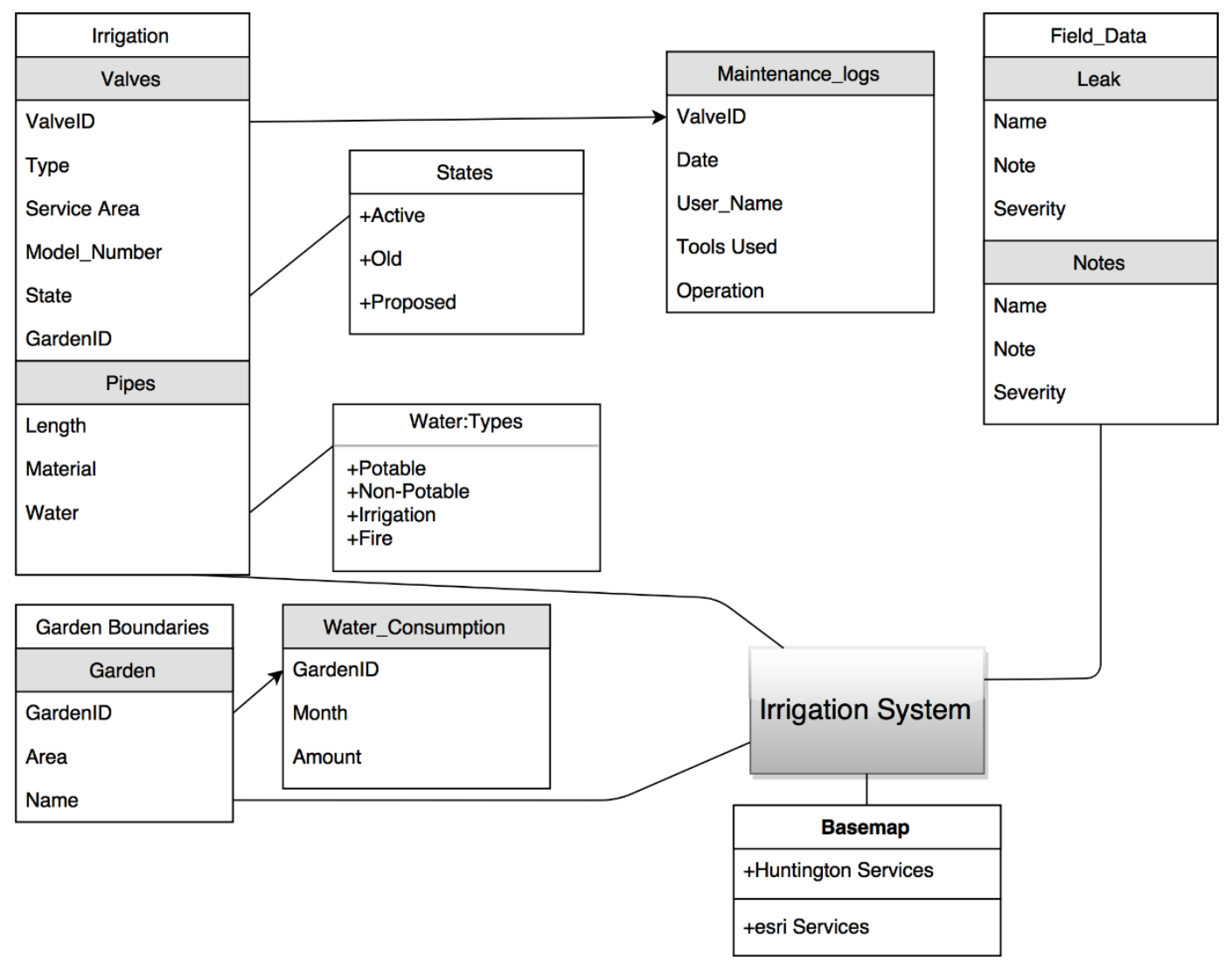

Figure 4.2: Irrigation System Logical Model

Within the geodatabase were three primary feature datasets: irrigation, gardens, and field data. These datasets included valves and pipes, garden boundaries, and the leak notes, respectively. There is a join between the water-consumption table and the garden features based on the gardenID attribute, as well as between the maintenance table and the valve features based on the valveID attribute. Domains are in place for the water types, the states of the valves, and the severity values.

Within the valve features, the valveID is a unique identifier, which utilized a naming convention of V1XXX where X is the variance in the ID. The V represents the type of feature, in this case a valve, and the single digit 1 allows for future inclusion of valves of another water system, at the discretion of the client. The type of valve is displayed by its type attribute: whether it is just a simple shut-off valve or a wireless control valve. The service area is the surveyed area that the valve supplies water. Model number is the number present on the valve, recorded during survey of the valves. State is the current operating condition of the valve: active, old, or proposed. Active means the valve still functions and is part of the system; inactive means the valve disconnected or removed. Proposed means the valve does not yet exist. The garden ID is the name of the garden the valve is controlling the flow to; this value will only be present on valves that control the flow from the mainline into the garden specific lines.

The pipes contain values associated with their material and their length, as well as the type of water contained within the pipes. The notes and leaks feature classes both contained attributes optimal for data collected in the field, such as who collected it, date collected, and how severe the leak or damage viewed was. 
The maintenance logs table has attributes that allow for easy records of when valves undergo maintenance and the problems associated with them. Collected attributes, such as who has worked on the valve and tools used in the past, gives a sense of who has experience with that valve and how often repairs on the valve occur. The water consumption table shows the consumption of water at the garden scale within a given month, allowing a view of consumption over time.

\subsection{Data Sources}

All data used in this project were obtained from the data management at the Huntington Library in the form of CAD drawings and PDF documents. These CAD drawings had all the features for pipe locations and valve locations on the site, including those used for non-irrigation purposes. These data were the result of previous surveys and engineer's schematics from previous projects. The PDF document featured a map that provided the boundaries of the individual gardens.

\subsection{Data Scrubbing and Loading}

The CAD drawings were converted into feature classes within ArcGIS desktop. The data were cleaned to contain the attributes from the model. This resulted in a large number of null values, which were acceptable by the client's standards, as it was the intent of the client to do a survey to reassess the valves. Join and relates connectivity were created between features and the empty tables for maintenance and water consumption. The tables were attributed based on the logical model. Unneeded attribute fields inherited from the CAD drawings were then deleted from the valve and pipe features. The PDF Boundaries were geo-referenced and manually traced into a polygon feature class.

\subsection{Summary}

This chapter demonstrated the steps taken to construct the database, and the development process that was involved in constructing the models that formed the basis of the geodatabase. These models were discussed in order to show how the spatial relationships were interpreted and transferred to a final project application setting. These models created the framework that was used when the data was cleaned and loaded to the project database. 


\section{Chapter 5 - Implementation}

This project aimed to create a series of application components to assist the Huntington Library irrigation department employees to easily view, modify, and collect data about the irrigation system. The client already heavily utilized an organizational ArcGIS Online account, so this project uploaded the feature services to ArcGIS Online. The major components of this project were: the irrigation management application for user viewing and editing of features; the consumption analysis application for viewing and editing the related tables; and the damage report application. This chapter covers the steps of the implementation stage: publishing features (Section5.1) and developing the web application (Section 5.2). A summary is provided in Section 5.3.

\subsection{Publishing Feature Services}

This project utilized a number of feature services, split up based on their primary function. Several of these features contained related tables that were linked to points and polygons on a map. The features included garden boundaries, irrigation features, and the irrigation notes features. The garden boundaries feature service contained the digitized boundaries of all the gardens as polygon features, and a related table designed to contain the water consumption of each garden for each month. This table and feature used the garden_ID attribute to create the one to many relationship. The irrigation features service contained the valve and pipe features, as well as the valve maintenance logs. The valve_ID attribute was used to create the one to many relationship between the valves and the logs. Within the irrigation notes feature service are point features that report leaks or other notes of interest. This service contained no related tables. Publishing these services required the use of ArcGIS desktop and an organizational ArcGIS Online account. Symbology and relationships were all assembled and managed in ArcGIS Desktop before uploading to ArcGIS Online, allowing a smooth transition into the webmap creation phase. Figure 5.1 shows the editing capabilities enabled for all feature services.

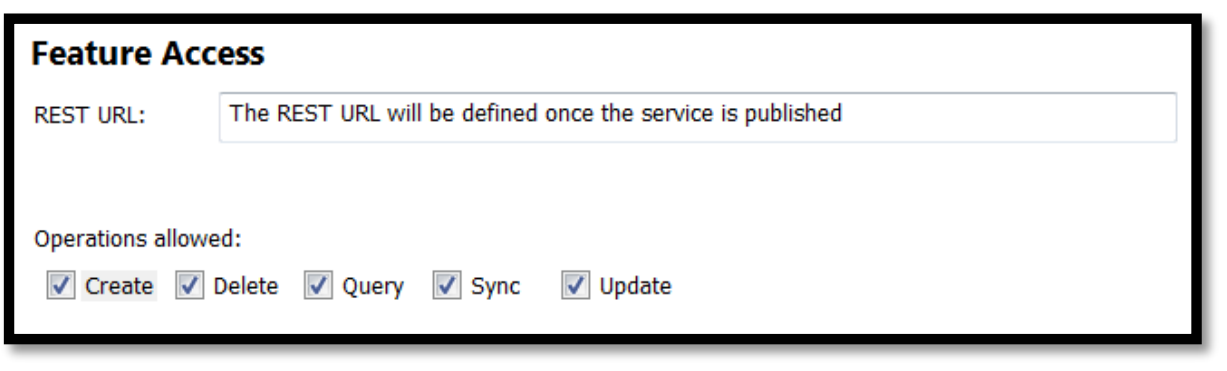

Figure 5.1: Feature Access Settings

When the feature services were published, sharing was configured based on what function the services needed to perform, and the security needed for the contents within. The boundaries and irrigation consumption of the gardens were secured by using the ArcGIS identity management tool, requiring a user to have an organizational account with the Huntington Library. This information was secured because the location of pipes 
and future work were deemed sensitive, but necessary to be shared within the organization so that a user within the organization could work with the application. When a new user needs authorization to use the application, the client simply needs to create an authorized organizational account for the new user, rather than having to deal with a custom secure login system. The irrigation notes needed to be publicly accessible, so that gardeners on-site could be given the editing application in order to mark leaks that they locate. This element of the system does not use a login, because it is a public application intended to be used by gardeners or other individuals who do not have organizational access. There was not a high demand from the client for these data to be very accurate, so the lack of control over who can edit features was acceptable for the applications purpose of capturing the general areas where damage has occurred. Figure 5.2 summarizes the feature layers and their elements.

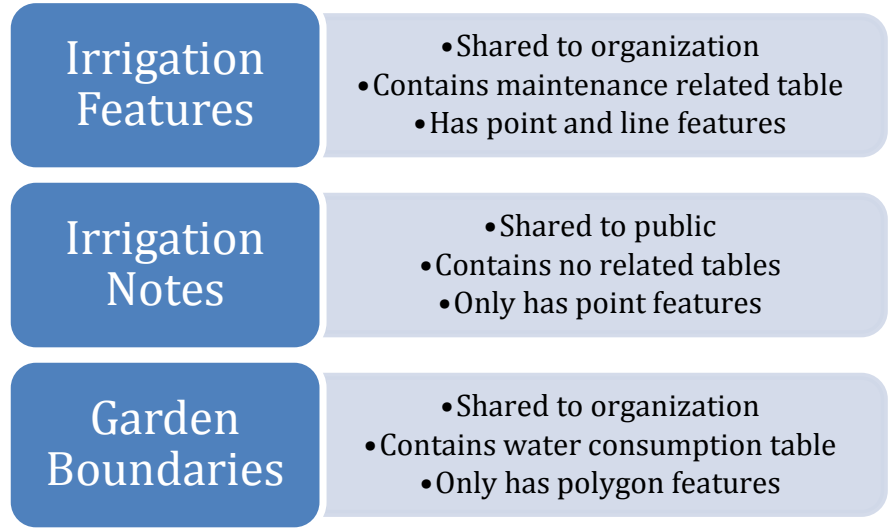

Figure 5.2: Feature Services Summary

\subsection{Creating the Web Applications}

Three components of the web application were built into this project: the client-editing app, the table-viewing app, and the public reporting app. The client-editing app was built using Esri Developer Web App Builder; the table-viewing app and the public reporting app were built using the ArcGIS API for JavaScript. JavaScript and Web App Builder accomplish different goals and optimize their functions based on the device most likely to be used for the task. Since all of these applications access the same feature layers hosted on ArcGIS Online, these applications are able to view the of other applications when the map itself is reloaded. The map loads from the server whenever the user pans the map or zooms, requesting a new map. This creates a connection between all the applications at their data source.

\subsubsection{Authoring Web Map}

The client needed to have a central location to view all the feature layers at the same time. The Web App Builder Application would fulfill this requirement by taking an authored web map and displaying it to users. Authoring the web map to contain all the hosted feature layers, and allowing editing of each feature accomplished this. The ability to edit would allow the client to adjust any of the features contained on the map. 
The web map featured an imagery base map hosted by the Huntington Library on their organizational account and the features published to ArcGIS Online by this project. An example of this web map is shown in Figure 5.3.

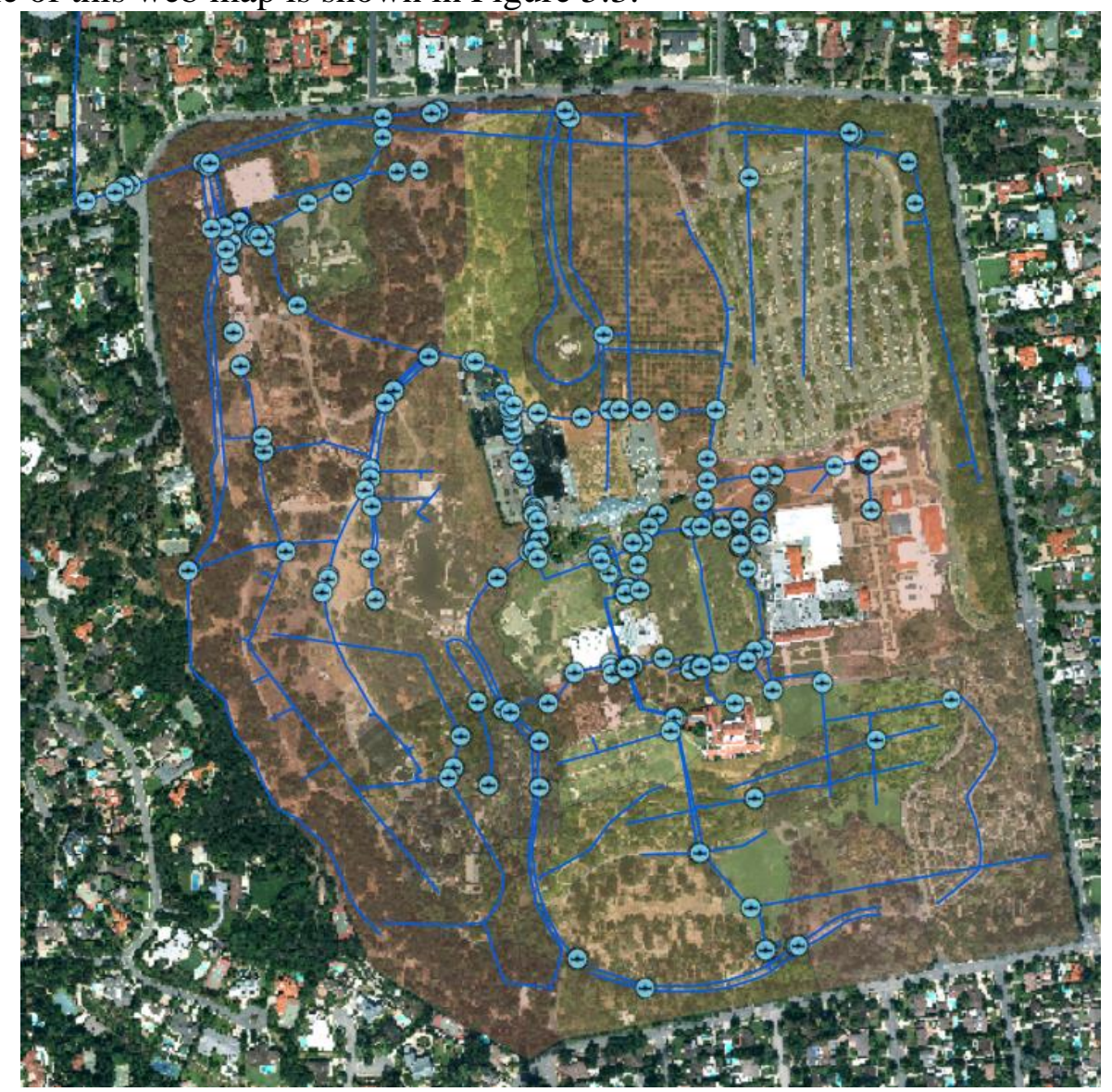

Figure 5.3: ArcGIS Online Web Map

After testing the edit functionality and insuring all features were in the web map, it was shared to the Huntington Library organizational account. The map was then published to the organization ArcGIS Online account, where it was updated with appropriate metadata. This included a description and tags, so that other users within the organization would know the purpose of this web map.

\subsubsection{Feature Editing Application}

The overall structure of the application was designed to be simple and easy to use, while still offering a full suite of options for modification and viewing of the data. The application also features a link in the upper right to the table viewing application. The overall structure of the application is shown in Figure 5.4. 


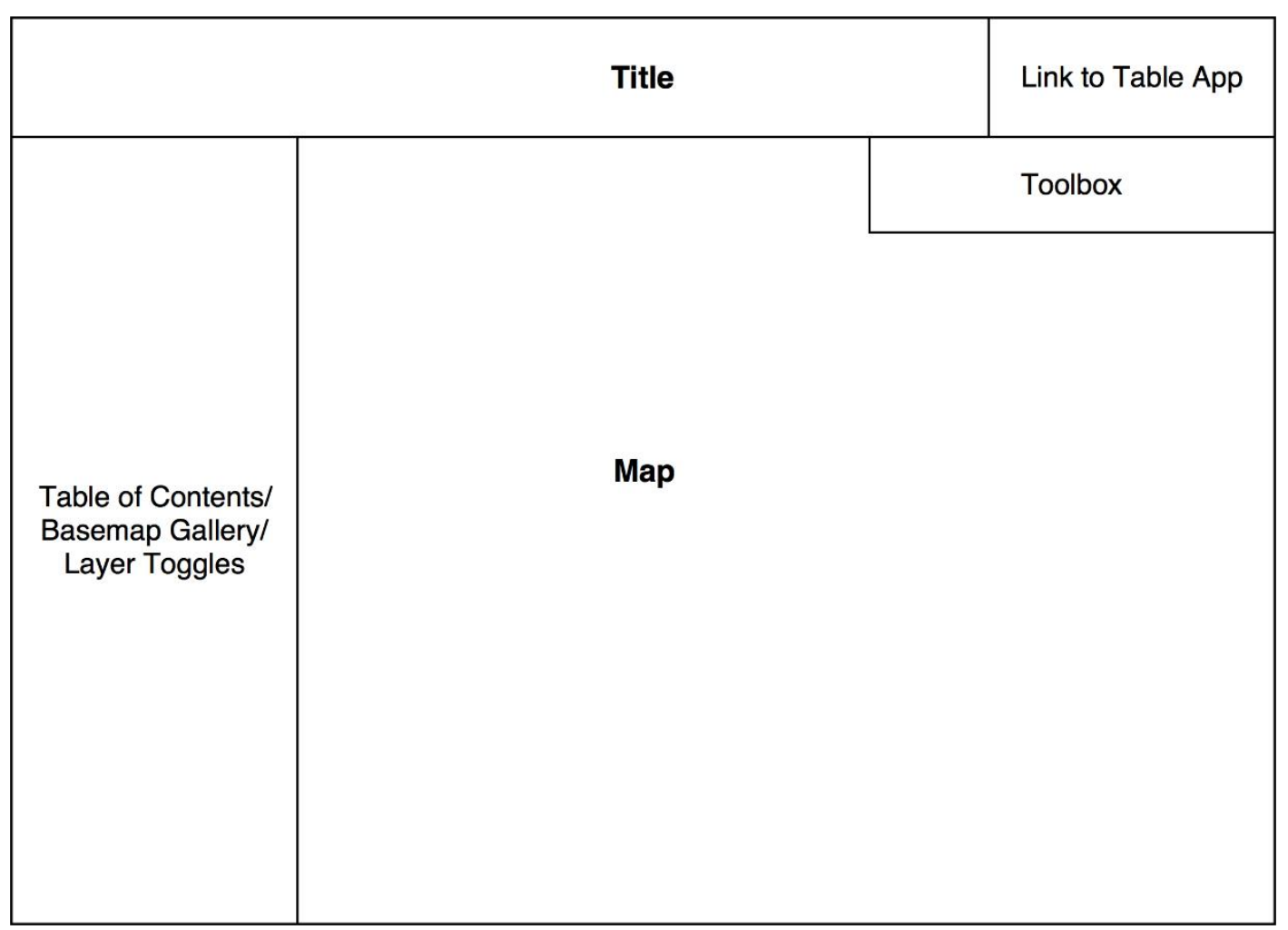

\section{Figure 5.4: Feature Editing Application User Interface Design}

The Developer Web App Builder was used to build the application for the client to view all the data and make edits. The simple, user-friendly interface of this tool was intended to allow easy access to the widgets the client would commonly use. It also allowed the client to reload the application in the Web App Builder to make changes to the customization of the application. This allows the client to make changes in the future, without having to dig into the coding. Another added benefit was that prior to this project, Esri had recently updated the Developer Web App Builder to support displaying related tables attached to a feature on the map. This allows a user to view the complete data for the related tables, and to filter the related table by the selected features. This functionality is shown in Figure 5.5. 


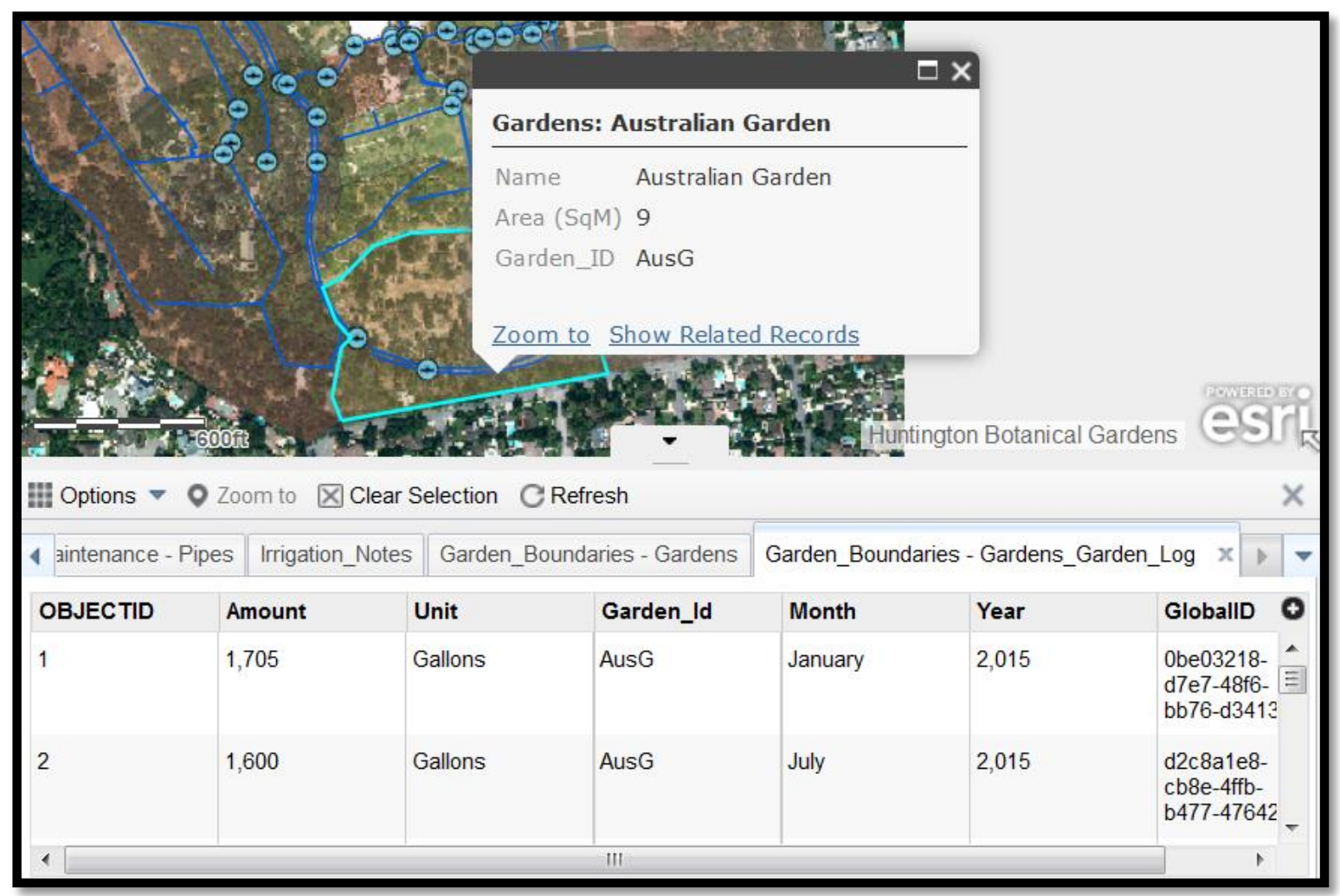

Figure 5.5: Related Records for a Selected Garden

This application was configured to display the legend, to toggle visible layers, and to change the basemap. These basic functions were implemented to allow the user to modify their view based on their current task. The user can easily toggle off layers they do not wish to currently edit in the toggle layers tab, which turns off the layer visually as well as hiding those feature templates in the editor widget. This prevents the user from accidentally editing an unintended garden polygon, or valve. In the upper right are widgets designed for working with the features. Shown in figure 5.6 the editing toolbar allows a user to add new features of any type, as well as delete or edit the attributes of features already on the map. The measurement tool can measure the distances between features or the area of a site, and the query tool was configured to allow the user to easily search for valves by their ID, also shown in Figure 5.6. 

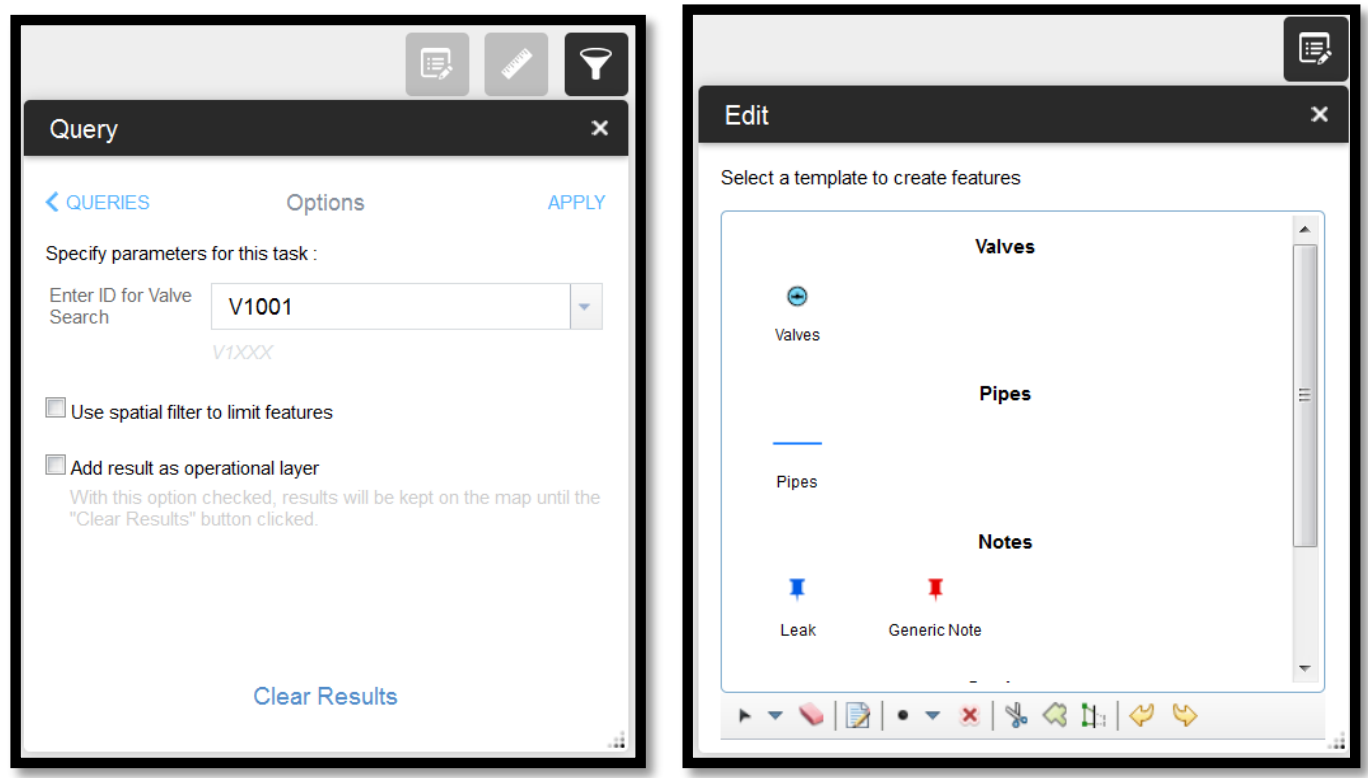

Figure 5.6: Valve Search Query and Edit Box

\subsubsection{Water Consumption Visualization Application}

The water consumption application was written in JavaScript to work primarily with the related tables within the feature services. The overall design of this application is shown in Figure 5.7. In the center of the application is the map, constructed from the garden boundaries and irrigation features service.

\begin{tabular}{|c|c|}
\hline \multicolumn{2}{|c|}{ Title } \\
\hline Map & $\begin{array}{c}\text { Application Tool Tabs } \\
\text { Legend/ } \\
\text { Charts/ } \\
\text { Tables/ } \\
\text { Add Consumption/ } \\
\text { Add Log }\end{array}$ \\
\hline
\end{tabular}

Figure 5.7: Water Consumption Visualization App Design 
In this application, the garden names are labeled so that the viewer can easily identify the garden regions based on the boundaries. Along the right side of the application are the accordion style tabs, which allow users to cycle through the different functions of the application. The tabs are legend, charts, tables, and edits. The legend shows the features that have been added, and their symbols. It is automatically updated with new garden features that are added, without needing to edit the code, shown in Figure 5.8.

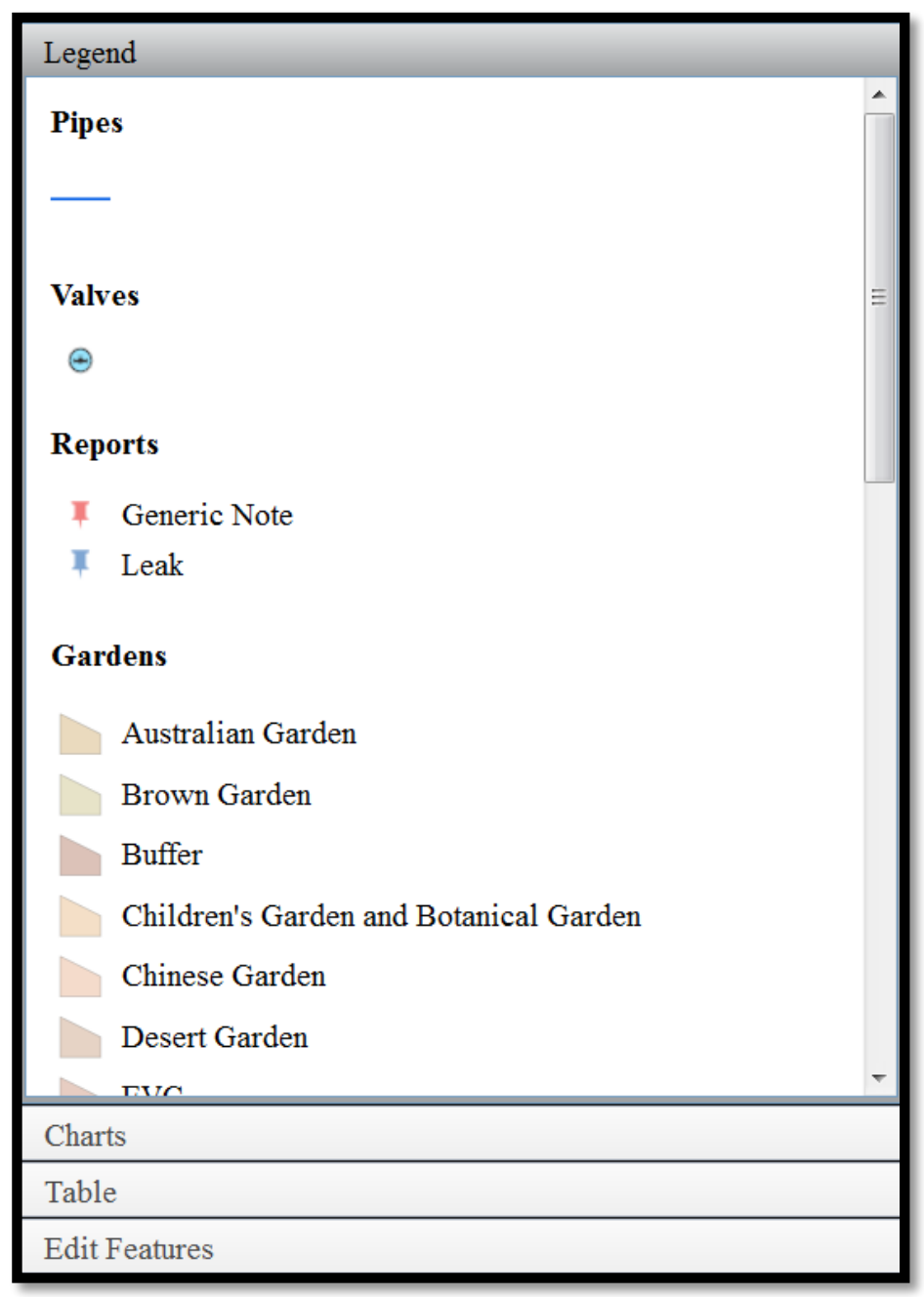

Figure 5.8: Layer Legend under the Legend Tab

Under the chart tab, a user can visualize a garden's yearly water consumption as a line chart as shown in Figure 5.9. When a user selects a year in this line chart, the consumption values for each month of the year are displayed in a bar chart. This allows the user to observe differences in years for the gardens, and even compare certain months visually. This can be combined with maintenance logs and knowledge of the organizational policy or watering changes in that year, to allow better oversight on how their choices are impacting the gardens. A user can choose a garden by clocking on the map or from a dropdown menu. 


\section{Select Garden: Australian Garden}

\section{Chart Comparing Consumption of Water Per Year for Selected Garden}

90,000

86,000

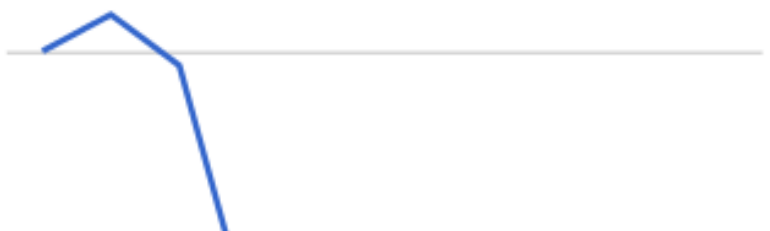

82,000

78,000

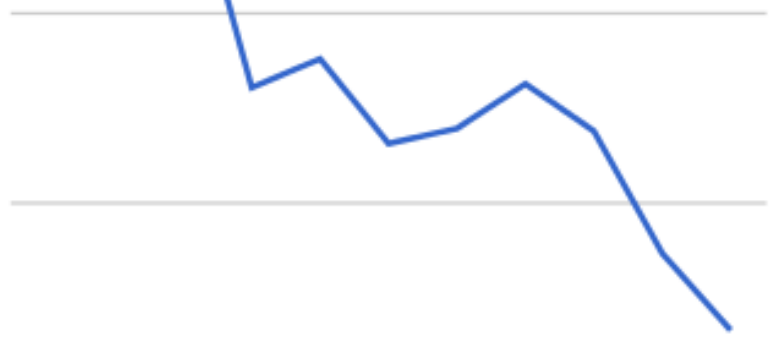

74,000

$\begin{array}{ccccccc}2004 & 2006 & 2008 & 2010 & 2012 & 2014 \\ 2005 & 2007 & 2009 & 2011 & 2013\end{array}$

Figure 5.9: Demonstration of Line Chart Showing Water Consumption for Australian Garden

The workflow of generating charts is shown in Figure 5.10. It starts with queries on the water consumption table and the returned results are the monthly water consumption. It then runs through and adds all monthly consumptions together within the year. It is displayed using Google Visualization API. The application listens for when the line chart is clicked, it then retrieves and displays monthly consumption for that year.

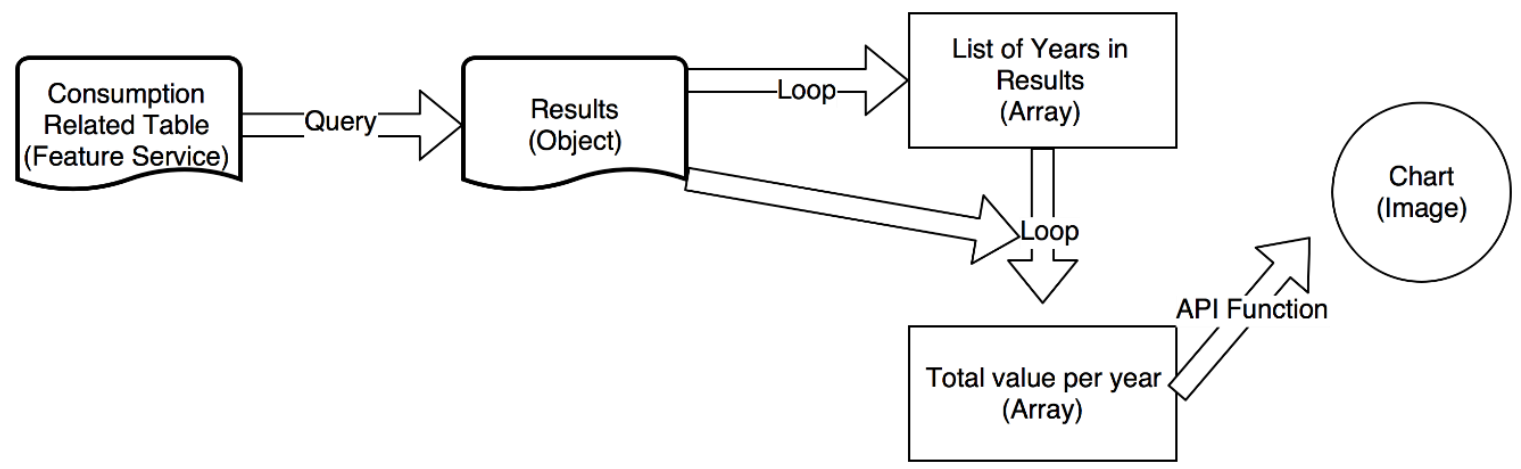




\section{Figure 5.10: Chart Workflow}

This method is applied again for the table access tab. Which contains a table of maintenance logs for the selected valve. Selecting a valve triggers a listener on the layer, which causes the value of the selected valve to be sent to a table query. This pulls in the table and iterates using a loop, assigning the variables to arrays containing attributes to display in a table. It was noticed that the value returned for Esri date fields was a strange string of numbers. This had to be run through the Dojo data tool to convert it into a readable date. An example of such an output table is shown in Figure 5.11.

\section{Table}

ID

$$
\text { date }
$$

Tool

1 V1052 March 14, 2015 Hammer

2 V1052 April 19, 2015 Hammer

\section{Figure 5.11: An Example of Maintenance Logs Shown in Table}

The final feature was the ability to add new features to the related table, or to search by objectID and edit the attributes of a feature in the table. The feature is added by assigning all the values of the user input into an array, and then delivering that array by using the apply edit command from ArcGIS API for Javascript. This allows the user to modify the related table, a feature previously only available in ArcGIS collector. This is important for easily adding new values for the month or fixing wrongly entered information, without having to reset the entire dataset, or uploading the table again from desktop. 


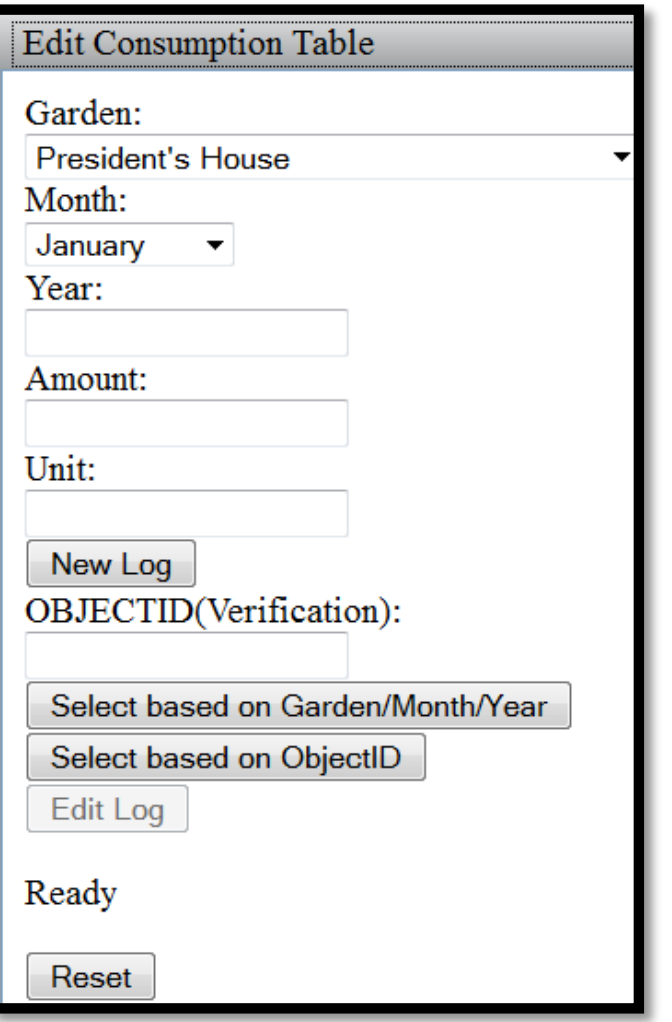

Figure 5.12: Edit Table Window

When the user has not yet done a search for an already existent log, the Edit Log button is disabled. Once the user has completed a search, the New Log button becomes disabled, and the Edit Log button enabled. This is to insure the user does not accidentally enter a duplicate value, when they meant to change a value. If the user decides they have made a mistake, a Reset button clears all the boxes, and sets the buttons to their default state.

\subsubsection{Damage Report Application}

The reporting application was developed with the ArcGIS API for JavaScript. This application focused on collecting data from less experienced GIS users such as gardeners or visitors. These users may struggle to report issues utilizing a paper map, so this application allows them to find their locations via the geolocator tool, using the GPS built into the phone. They can then easily add a point on the map just by clicking the report type, and then clicking on the map. The user can then use dropdowns to add information, and customize information via textboxes. This application is shown in Figure 5.13. 


\section{IIIIrigation Report App}

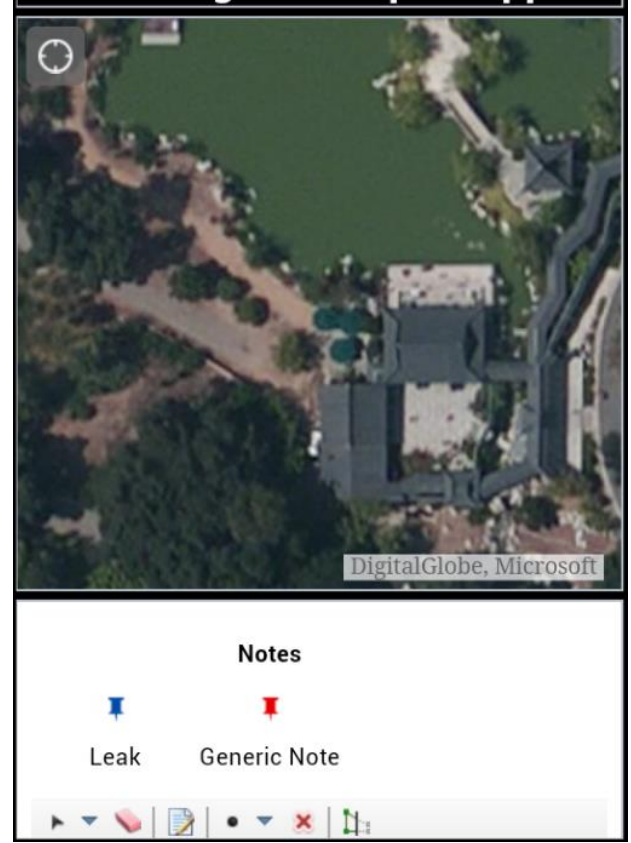

Figure 5.13: Mobile Damage Report Application

\subsection{Summary}

In this chapter, the features and functions used to create the applications were covered. Section 5.1 covered the publication of the feature services to ArcGIS Online. Section 5.2 covered the creation of the webmap, the management application, the consumption application, and the damage report application. These applications were developed using JavaScript and Developer Web App Builder. They share a common data source by accessing the same feature layers hosted on ArcGIS online meaning that edits and reports from the applications are shared amongst them. 



\section{Chapter 6 - Use Cases}

This project was implemented so that the client would be able to visualize data scattered across multiple sources within a single system. This allowed the client to observe these data, and make important choices in regards to the irrigation system. They can decrease water consumption while still maintaining the beauty and diversity of Gardens. This chapter focuses on the results of this project by covering the potential use cases of this application. Each section in this chapter covers the use cases specific to each application: Section 6.1 covers the uses of the management application, Section 6.2 covers the uses of the consumption application, and Section 6.3 covers the uses of the damage report application.

\subsection{Use Case: Adding New Valves and Pipe Using Irrigation Management Application}

The irrigation management application's primary functions focused on allowing a user to edit features, view the data available to them, perform measurements, and search for valves by ID. This application also allows a user to edit the layers in the map by modifying the webmap hosted on ArcGIS Online. The overall view of this application is shown in Figure 6.1.

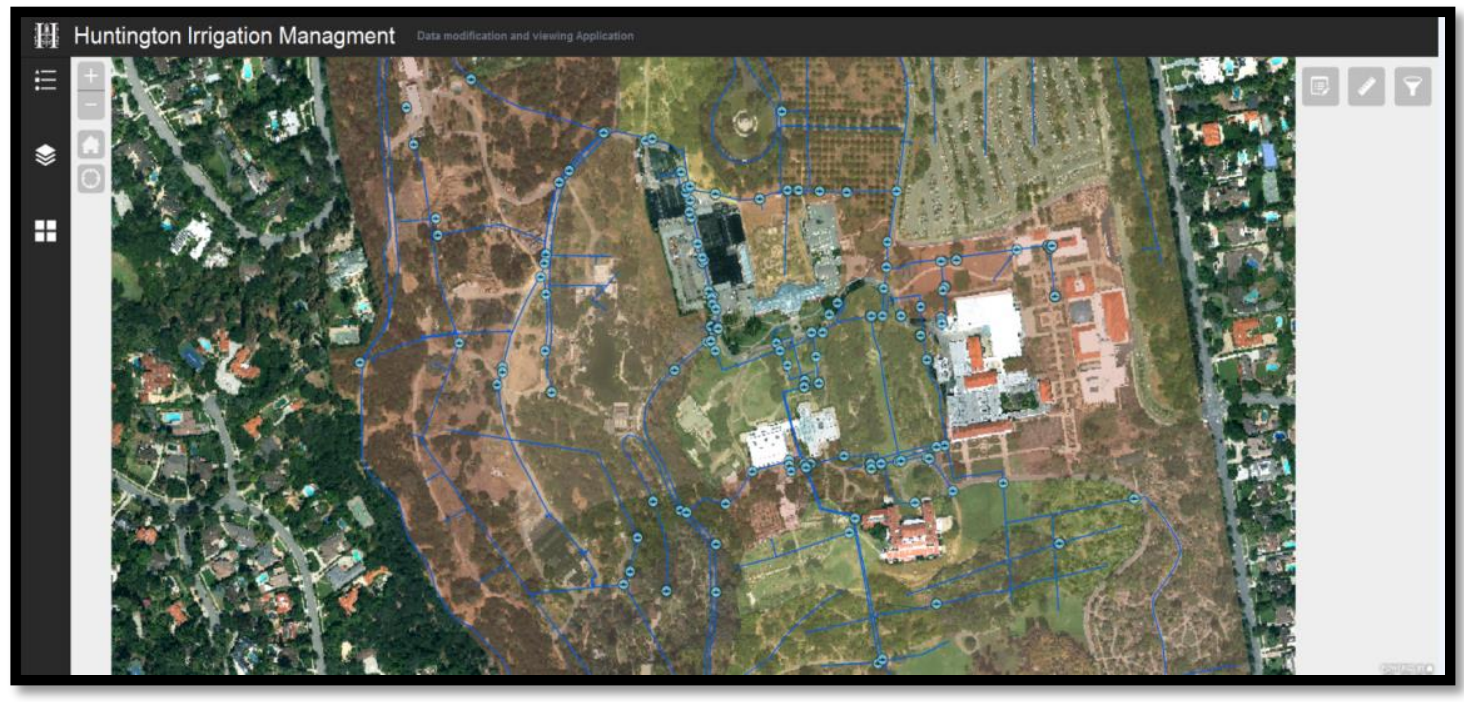

Figure 6.1: Irrigation Management Application

In this use case, a user has been tasked with adding a new pipe connecting two valves, as well as adding a new valve at the end of a planned piece of pipe. When booting up the application, the first action the user takes is to open up the layer list. It is unnecessary to have the screen cluttered with all the boundaries of gardens, as well as the points of all the notes that people have reported.

The user selects the stacked layer symbol to the right of the screen, and easily unchecks the irrigation note layer, as well as the garden boundary layer, which quickly reduces the amount of clutter on the screen, as shown in Figure 6.2. Next, the user knows 
that one of the connections for the new pipe is at valve V1052, and searches for this valve to zoom to the site.

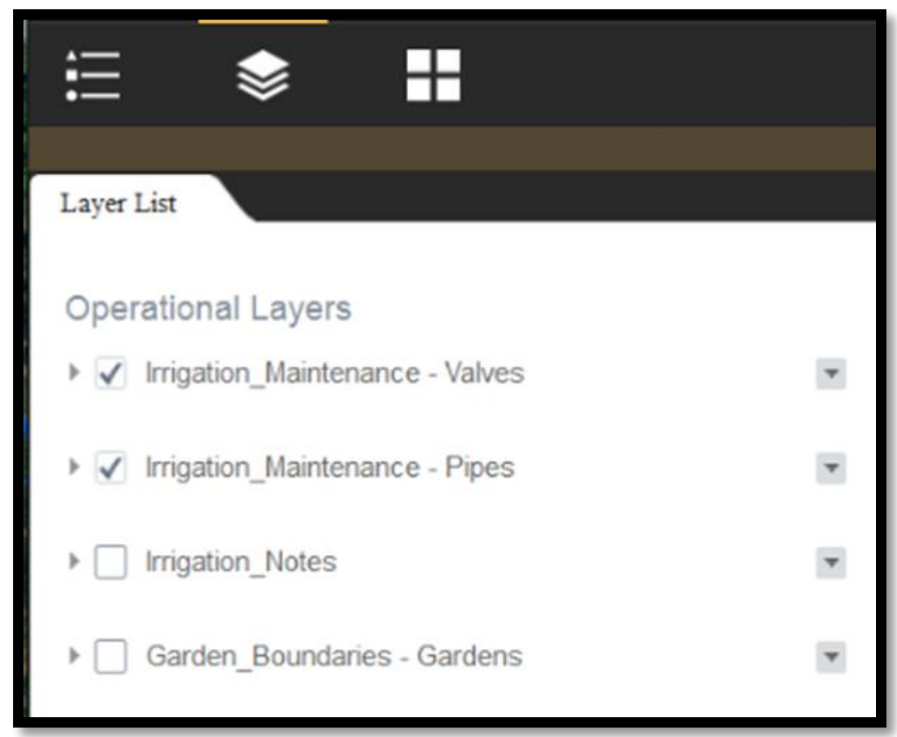

\section{Figure 6.2: Hiding Layers}

The user opens up the query tool by clicking on its button in the upper right collection of tools, then selects the search for valve by ID option and types in the valve ID. The user presses Apply, and the query moves the map centered at the location of the valve. The valve is highlighted on the map and its attributes are displayed in the query box. This result is shown in Figure 6.3.

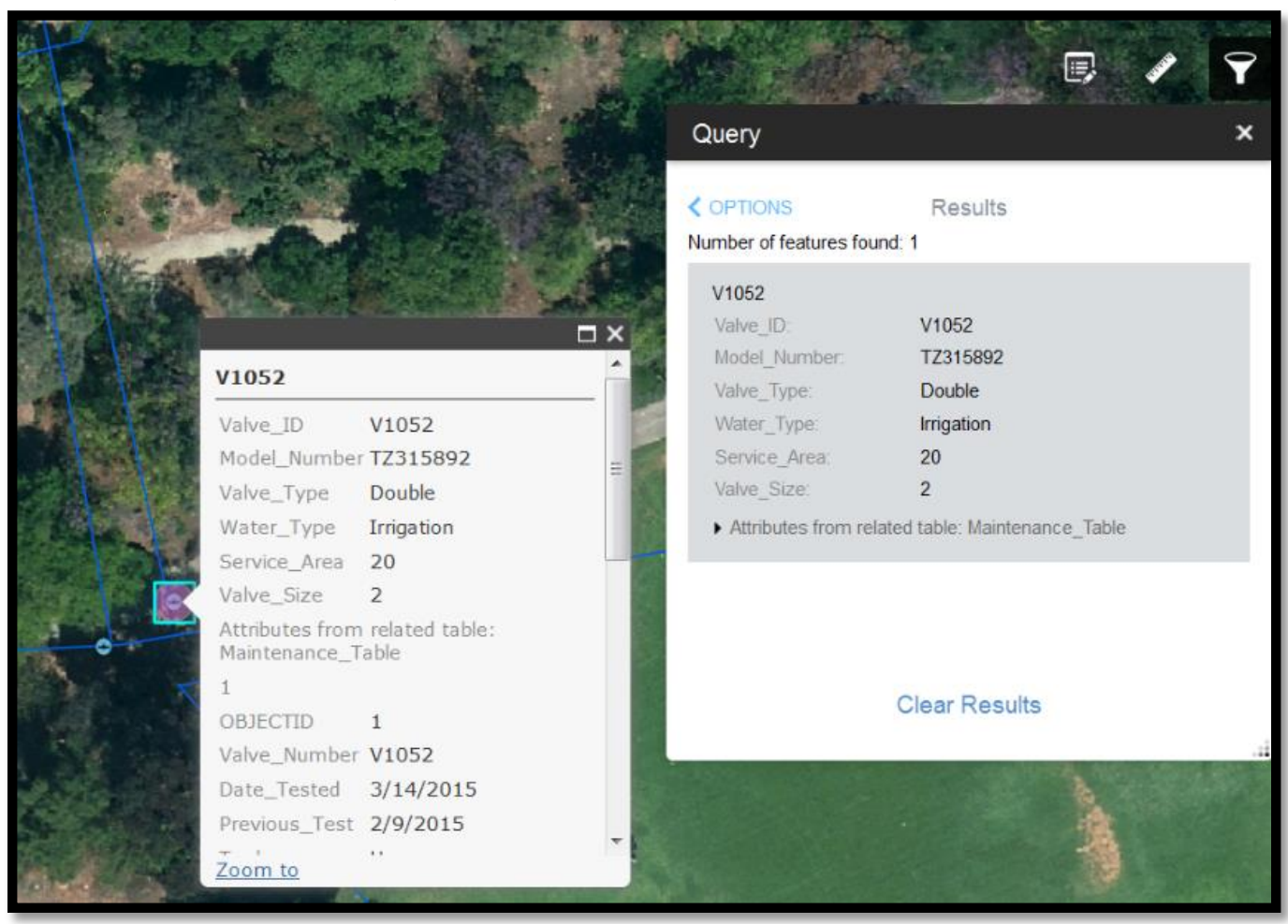

Figure 6.3: Results of Search 
Once the valve is found, the user is able to check if the distance between the two valves matches the distance in the request, which is about 6.5 meters. The user does this by selecting the measurement tool, and then clicking on both ends of the line to be measured.

After getting the result, the user concludes that 6.51 meters is close enough, if the number given was instead in feet the user would be able to click the small arrow next to meters to switch the units of measurement to feet. This result window is show in Figure 6.4 .

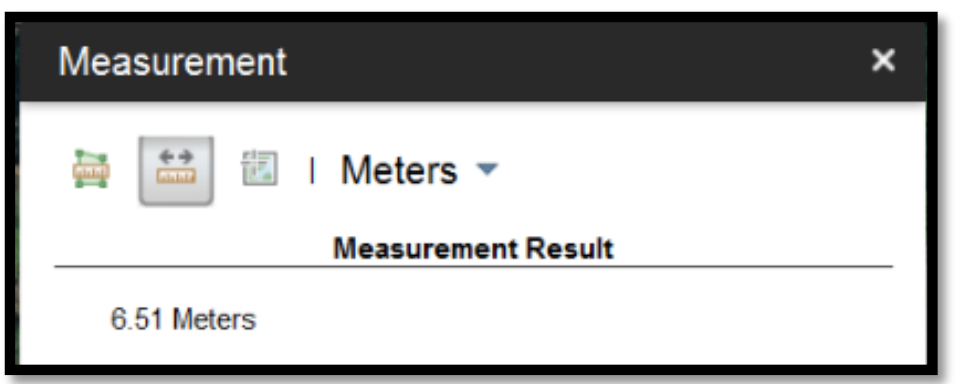

\section{Figure 6.4: Measurement Results}

The user is now ready to add the new line to the map, so the user clicks on the edit tool in the upper right, and bring up the edit dialogue, shown in Figure 6.5.

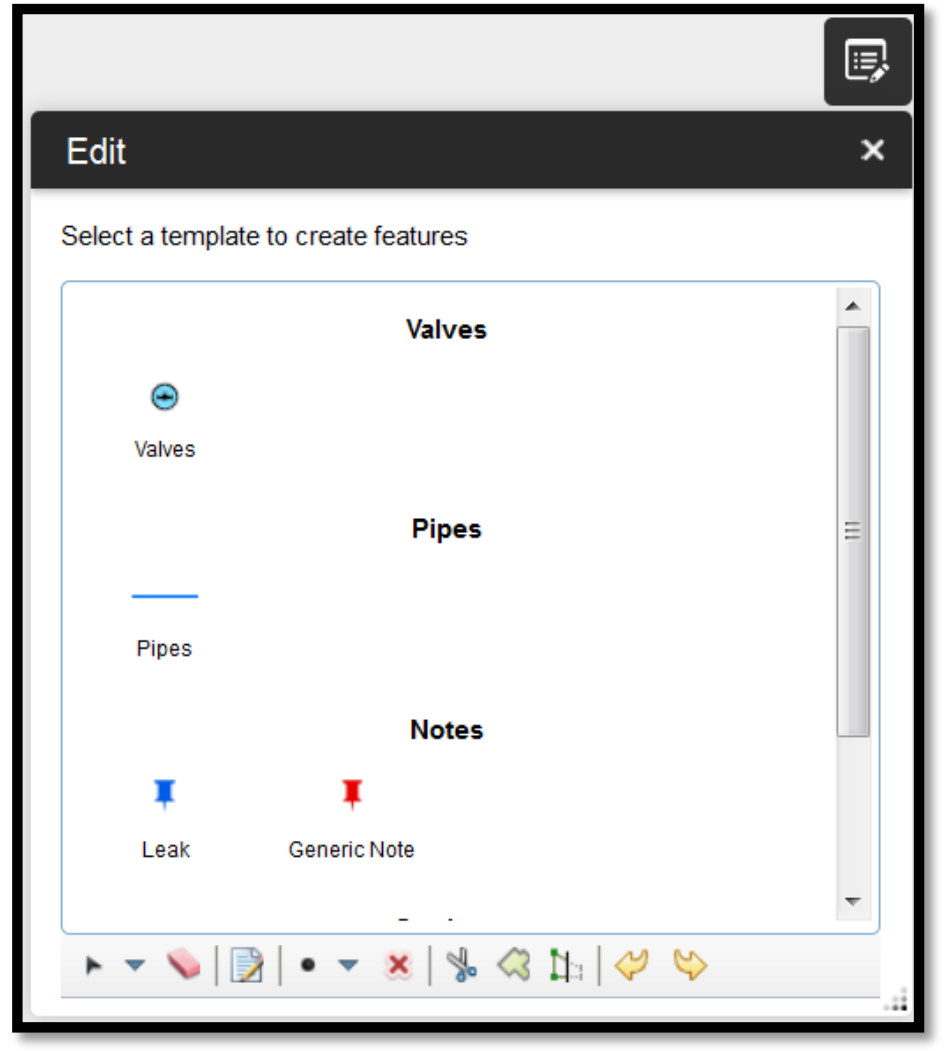

Figure 6.5: Options for Features in the Edit Window 
In this dialogue box, the user can see all the possible features that can be added, and selects the new pipe feature and returns to the map, leaving the editing window open to remain in editing mode. The user then clicks the two points between which a new pipe is built, and double clicks to finish the operation. The edit operation is shown in Figure 6.6


Figure 6.6: A New Pipe is Built in the Feature Layer Using the Edit Operation

The last task the user has to complete is to add a new valve along a nearby road. In the editing dialogue the valve symbol is selected, and then clicked on the map to place the point.

After placing the point, the user is then able to edit the attributes of the feature, as shown in Figure 6.7. These text boxes also appear when selecting any existing point when in edit mode, allowing the user to update attributes of the features.

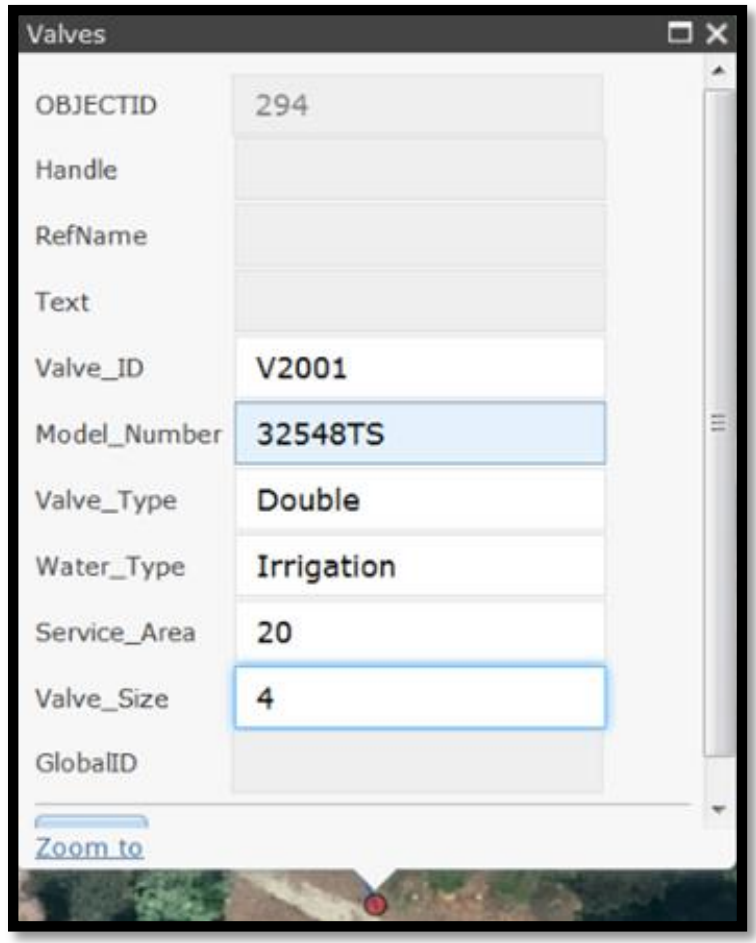

Figure 6.7: New Valve Attributes Window 


\subsection{Water Consumption Application}

The water consumption application allows a user to easily view data in the related tables in the form of line and bar charts for consumption, and in a table form for maintenance logs. The application also allows a user to add new records to those tables. This interface is shown in Figure 6.8. It also allows a user to view attributes of the layers displayed on the map. Should a user need to change layers within this application, they simply need to open the HTML file and place the new REST url into the location designated at the top of the code.

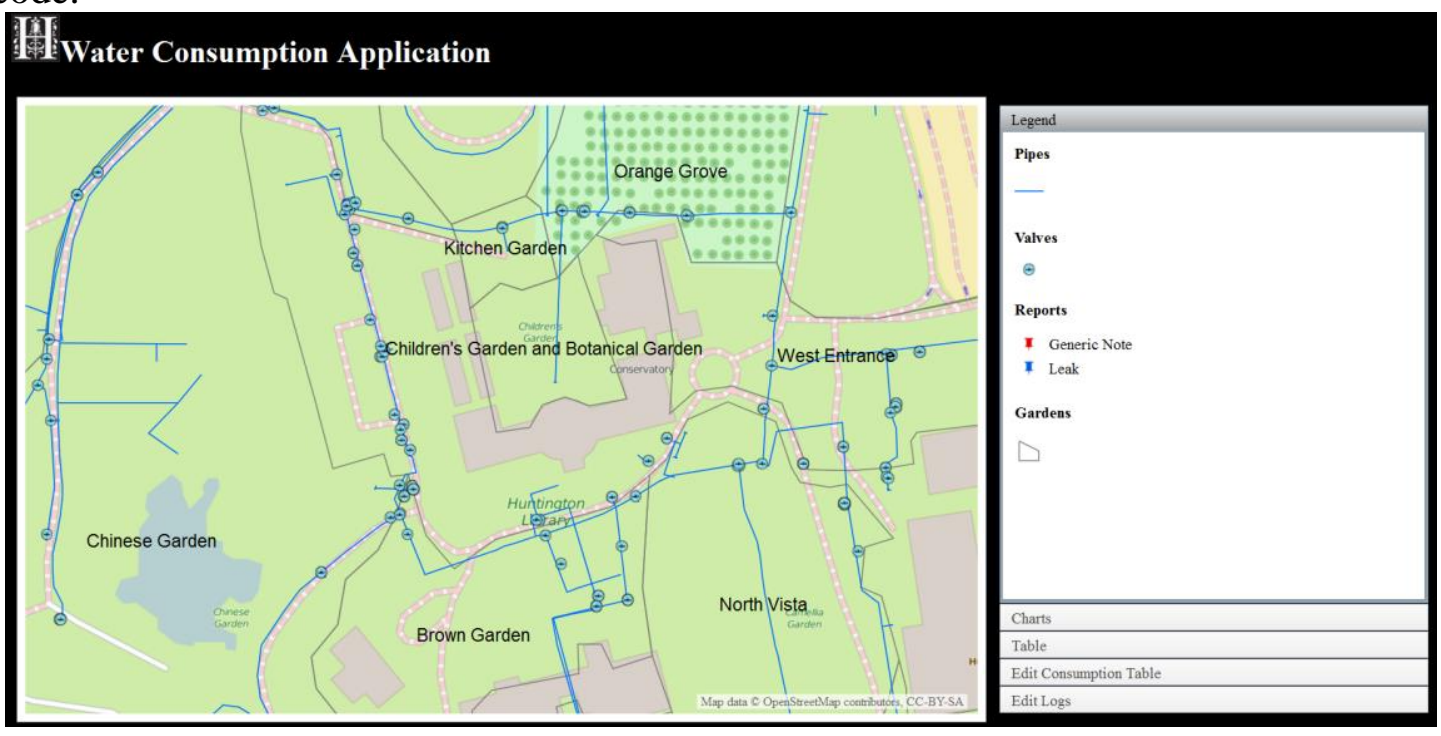

Figure 6.8: Water Consumption Application

\subsubsection{Use Case: Viewing Water Consumption for a Garden}

In this scenario, the user has been working closely with managers to reduce the water consumption of the Australian Garden. They want to check the consumption logs to see if that is the case, or if further efforts are needed.

The user finds the Australian Garden by looking at the labels present in the map. Once found, they click the garden polygon, which selects it automatically from the drop down menu in the chart tab, even though they have not yet opened the tab. The window showing this result is presented in Figure 6.9. 


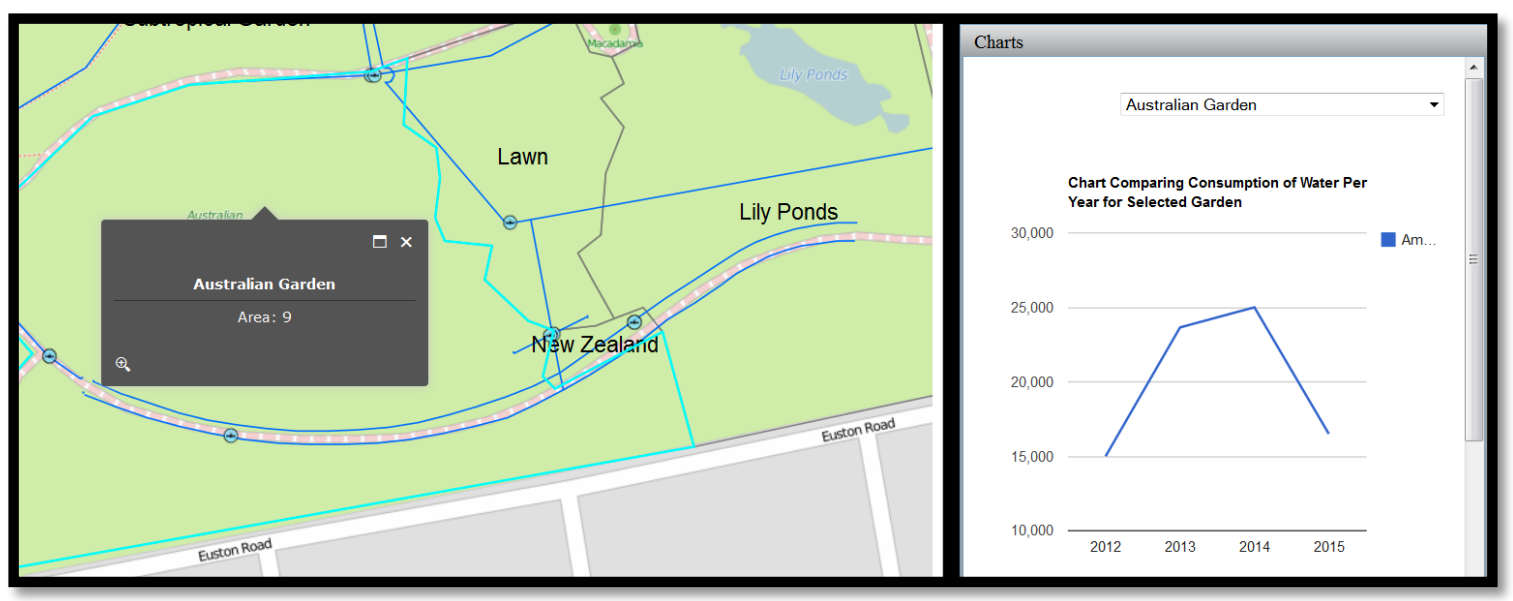

\section{Figure 6.9: Selected Garden and Line Chart}

The user then selects the year of consumption he or she wants to get a better look at by clicking on that year's point in the line chart, which will then display the values for all months in that year on a bar chart, shown in Figure 6.10.

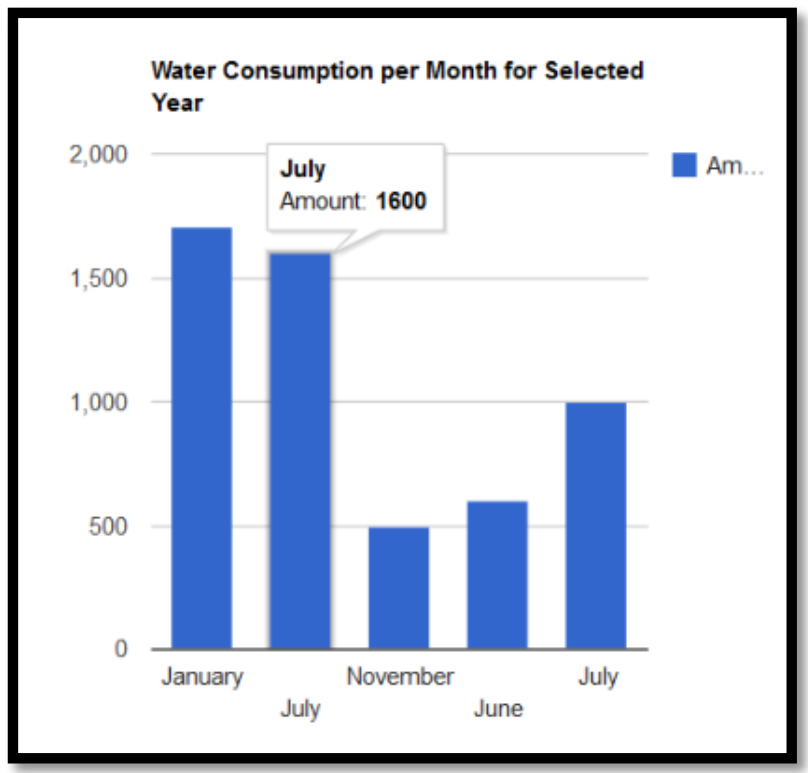

\section{Figure 6.10: Bar Chart Showing Monthly Water Consumption}

The user can highlight the bars to see the actual amount they represent. This also applies to the line chart created from the addition of all entries that year.

\subsubsection{Use Case: Valve Maintenance Logs}

In this case, a user is attempting to see a valve to look at the maintenance logs, and to add the most recent log. The user selects a valve, which brings up a pop-up window that shows the attributes of that valve, shown in Figure 6.11. 


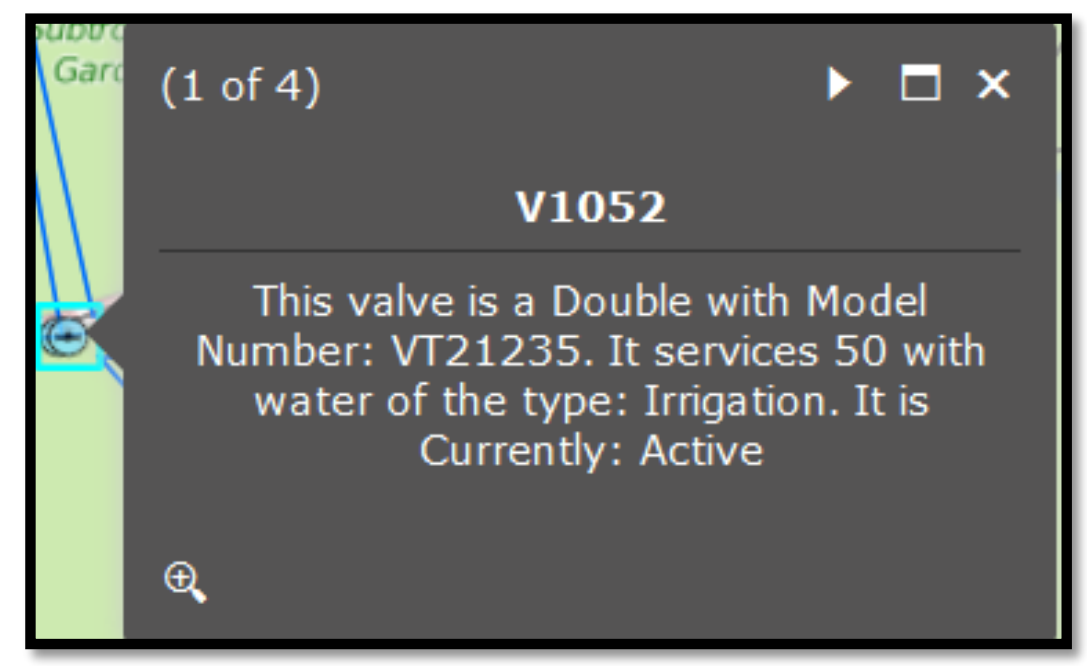

Figure 6.11: Pop-up Window Showing Valve Information

From this, the user confirms it is the correct valve and selects the Table tab to view the maintenance log table. The log table is shown in Figure 6.12.

\begin{tabular}{|c|c|c|c|}
\hline \multicolumn{4}{|c|}{ Table } \\
\hline & ID & date & Tool \\
\hline 1 & V1052 & March 14, 2015 & Hammer \\
\hline 2 & V1052 & April 19, 2015 & Hammer \\
\hline 3 & V1052 & July 1, 2015 & Wrench and Hammer \\
\hline
\end{tabular}

\section{Figure 6.12: Maintenance Table for Selected Valve}

When the user brings up the table, they can see that there are only two logs of maintenance on this valve. They then take the most recent report from another file or paper source and prepare to enter the new record by going to the Edit Logs tab of the application. This tab is shown in Figure 6.13. 


\section{Edit Logs}

Valve Number:

V1052

Tools Used:

Wrench

Date of last Maintenance:

6/22/2010

Date of Maintenance:

6/5/2011

New Report

OBJECTID(For Search):

\section{Select ID}

\section{Edit Reports}

Ready

\section{Figure 6.13: Entering a New Log}

The user then inputs the new data into the relevant entry boxes, and when finished clicks the Submit New Feature Button. The application clears the input boxes and displays a "complete" message if the operation was a success. If the application fails the message box will display more information about the error, such as what field was entered incorrectly, or if the server did not respond.

Reselecting the valve will show the new record that has been added, shown in Figure 6.14 .

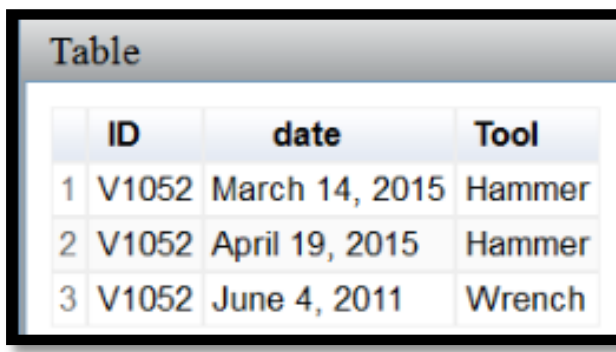

Figure 6.14: New Report Added

\subsection{Use Case: Update Water Consumption Information}

In this situation, the user has been tasked with finding the log entry for the Australian Garden in June of 2014, and changing the value of the amount consumed from 14080 to 15000. The user begins by navigating to the Edit Consumption Table tab along the right accordion. When this window is first brought up, all the fields are initially blank. If the 
user selects the Garden, Month, and Year clicking the Select based on Garden/Month/Year will search for that log. These windows are shown in Figure 6.15.

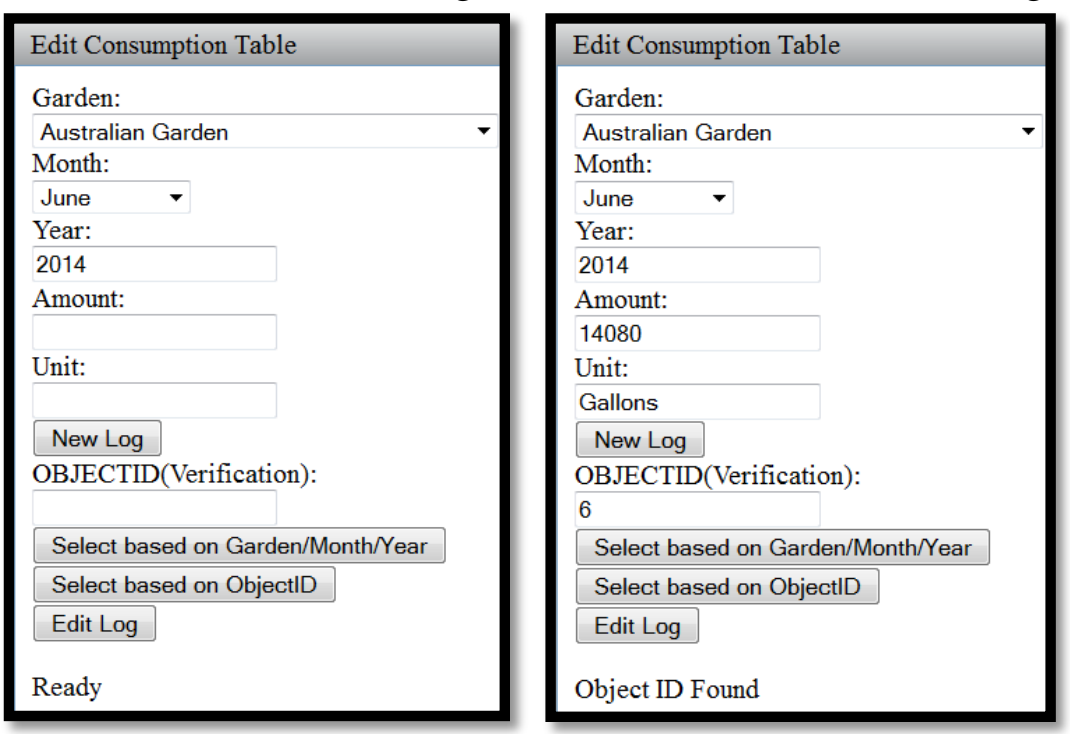

Figure 6.15: Searching by Garden, Month, and Year to Find Record

The boxes are filled with the remaining values from the report. Near the bottom of the screen, a message displays saying "objectID found". If there is no report with that objectID, the application returns an error message that there is no entry with those values. When the user changes any values inside of the box, and clicks the Apply Edits Button beneath Select based on ObjectID, it changes the values of that report to what is currently in the boxes. If any fields are empty, an error message is returned. If it is completed successfully, the entry fields are reset, and a complete message is displayed. This operation can also be completed if the user knows the ObjectID by entering the ID and clicking Search by ObjectID.

\subsection{Damage Report Application Use Case: Reporting a Leak}

The damage report application is designed to be simple and only displays locations with reported issues such as leaks. This application is designed primarily for use by a mobile device, but due to being a web application, it can also work on a tablet or phone. The interface for the Report Application is in Figure 6.16. 


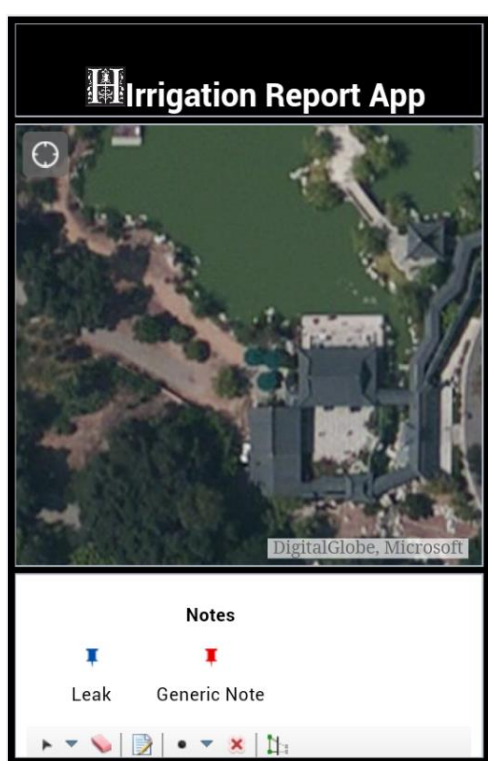

\section{Figure 6.16: Damage Report Application}

In this case, a user is working in the gardens, and sees that a nearby water socket is leaking. The user opens the report application to make note of its location for later repair. The user can click the geolocate button, and after giving permissions, the application will locate where the user is using the devices GPS.

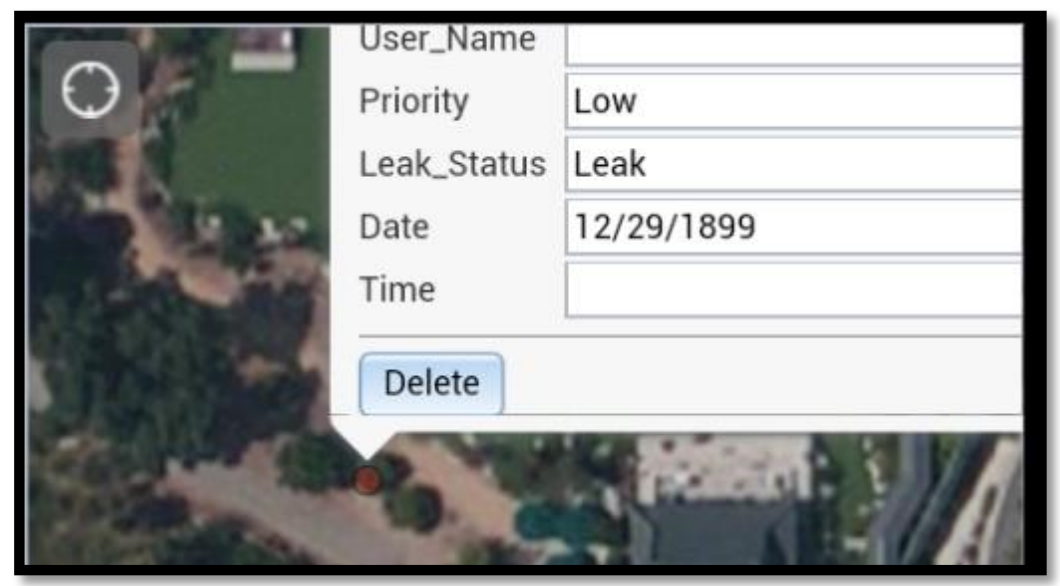

\section{Figure 6.17: Newly Placed Point}

The user can then select the leak type at the bottom of the screen, and tap on the screen. This will create a new point on the map. The user can then input values into the input fields. For the priority and date fields, there are selection tools to assist in selecting the correct value. 

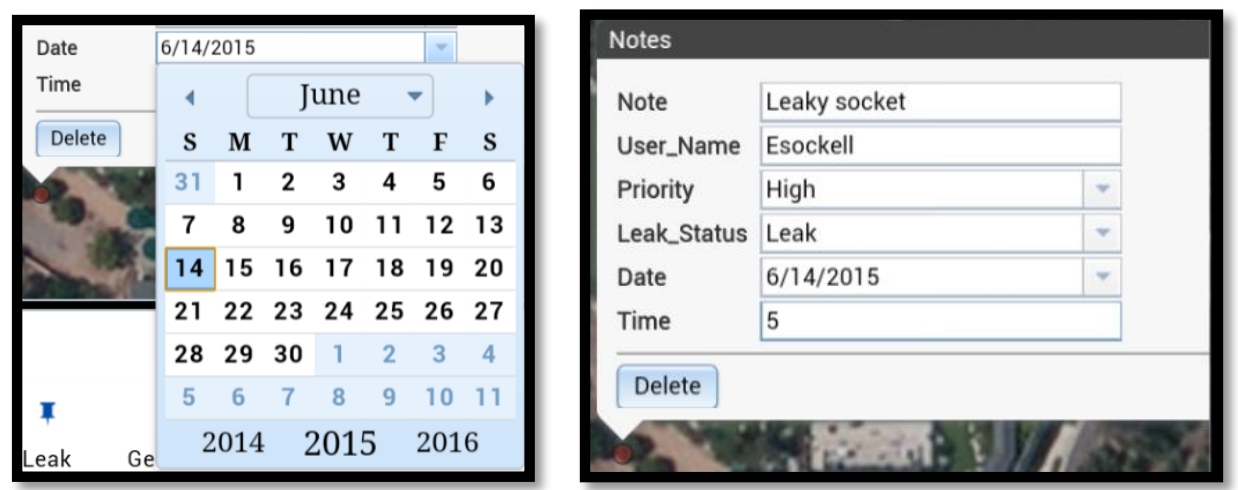

Figure 6.18: Date Selection Box (Left) and Filled out Values (Right)

This new point and its attributes are automatically added to the report feature layer online, and can be viewed by all the other components of this application. This allows individuals in the office to use these data to get a sense of what is going on in the field.

\subsection{Summary}

This chapter looked at use cases for all three major components of the application in this project. These uses cases covered the features of the applications and how they were designed to improve the client's workflow. 



\section{Chapter 7 - Conclusions and Future Work}

The irrigation management application allowed its users to add new features to any of the datasets within the webmap. Users can add new valves, pipes, reports, or modify the boundaries of the gardens. A user is able to search by valves to navigate around the map, and turn layers on and off based on the current view needed. Users can also add new layers to the web map such as plants or new data types in the future that may be collected by the Huntington Library without needing to modify the code. This application accomplishes the requirements to edit features and to observe the attributes of all features.

The consumption analysis application allows users to visualize data in the related tables using charts. A user can select a garden and see the water consumption change over time via a line chart, and select a year within that chart to view the more detailed bar chart showing monthly water consumption. This fulfills the requirement of being able to view water consumption data, and with the related table editing, also allows the user to manage these tables.

Finally, the reporting application allows a public user to report issues seen in the field without the need for an organizational log in. Users can easily use the geolocate function and their phone's GPS to locate themselves within the garden. This fulfills the client's requirement to collect data from the gardeners, who struggle to recall where they saw leaky pipes when asked to show where it was on a paper map. The field collection is public, and designed to accommodate phone web browsers.

The applications are in the following programming languages: JavaScript, Dojo, HTML, and CSS. These languages allowed customization of the presentation of the application, as well as the operating functionality of the application within a web browser. This application was tested and it functioned within the following web browsers: FireFox, Chrome, and Safari. The feature editing application was configured using Developer Web App Builder.

This project met the client's requirements, and has potential for future expansion. One of the opportunities for expansion would be to utilize real-time data streaming, allowing the application to access the valves on-site and listen to which valves are open or closed. This could also allow the application to get real-time information on how much water is flowing through the pipes currently. These data could be captured and allow constant updating of the consumption information for gardens, allowing daily updates on the current consumption for the month for each garden. This would allow greater control over the water consumption by gardens and would allow users to identify gardens that were consuming high amounts of water due to a possible leak.

This project was limited by the availability of API for the sensors valves used at The Huntington If the API for the valve system in use at the Huntington had been complete, it would have been possible to include live streaming of data to these applications, as well as allowing updating of the consumption data. This would have greatly improved the data accessible by the application, as well as the testing in how well the system can handle live data. The project was structured in a way that allows live data to be transferred into the feature classes at ArcGIS Online, but testing whether it will work with the valves at the Huntington was not possible. However, the methods and ideas in this project could 
easily be applied to smaller gardens, or larger gardens, looking to implement and begin centralizing data management using a GIS. These methods are also relevant to large agricultural institutions that already have systems collecting data, but want ways to view or centralize it. 


\section{Works Cited}

Booth, R., \& Rogers, J. (2001). Using GIS technology to manage intrastructure capital assets. American Water Works Association, 62-68.

Clark, M. (1998). Putting water in its place: A perspective on GIS in hydrology and water management. Hydrological Processes, 12(6), 823-834.

Durizzi, G. S. (2007). A GIS for Balboa Park's Desert Garden in San Diego, California (Master's thesis, University of Redlands). Retrieved from http://inspire.redlands.edu/gis_gradproj/28

Fortes, P. S., Platnov, A. R., \& Pereira, L. S. (2005). GISAREG-A GIS based irrigation scheduling simulation model to support improved water use. Agricultural Water Management, 77(1), 159-179.

Glissmann-Gough, S. (2005). Managing Plant Collections Using GPS/GIS in the Royal Botanic Gardens Melbourne (RBGM). Esri International User Conference.

Gogu, R., Carabin, G., Hallet, V., Peters, V., \& Dassargues, A. (2001). GIS-based hydrogeological databases and groundwater modelling. Hydrogeology Journal, 9(6), 555-569.

Halfawy, M. R., Pyzoha, D., \& El-Hosseiny, T. (2002). An integrated framework for GIS-based civil infrastructure management system. Proceedings of the Canadian Society for Civil Engineers (CSCE). Montreal, Canada.

Liu, J. (2009). A GIS-based tool for modelling large-scale crop-water relations. Environmental Modelling \& Software, 3(24), 411-422.

Lwin, K. K., \& Murayama, Y. (2011). Web-based GIS System for real-time field data collection using personal mobile phone. Journal of Geographic Information System, 3(4), 382.

Reichenbacher, T. (2001). Adaptive concepts for a mobile cartography. Journal of Geographical Sciences, 11(1), 43-53.

Satti, S. R., \& Jacobs, J. M. (2004). A GIS-based model to estimate the regionally distributed drought water demand. Agricultural Water Management, 66(1), 1-13.

Serre, D., Peyras, L., Tourment, R., \& Diab, Y. (2008). Levee performance assessment methods integrated in a GIS to support planning maintenance actions. Journal of Infrastructure Systems, 14(3), 201-213.

Shaner, J. (2015, February 18). Related Tables-Exploring New Ways to use Collector for ArcGIS. (esri) Retrieved from ArcGIS Resources: http://blogs.esri.com/esri/arcgis/2015/02/18/related-tables-exploring-new-waysto-use-collector-for-arcgis/

Tabesh, M., \& Saber, H. (2012). A prioritization model for rehabilitation of water distribution networks using GIS. Water Resources Management, 26(1), 225-241.

Tsou, M.-H. (2004). Integrated Mobile GIS and Wireless Internet Map Servers for Environmental Monitoring and Management. Cartography and Geographic Information Science, 31(3), 153-165. 



\section{Appendix A. Code for Viewing Application}

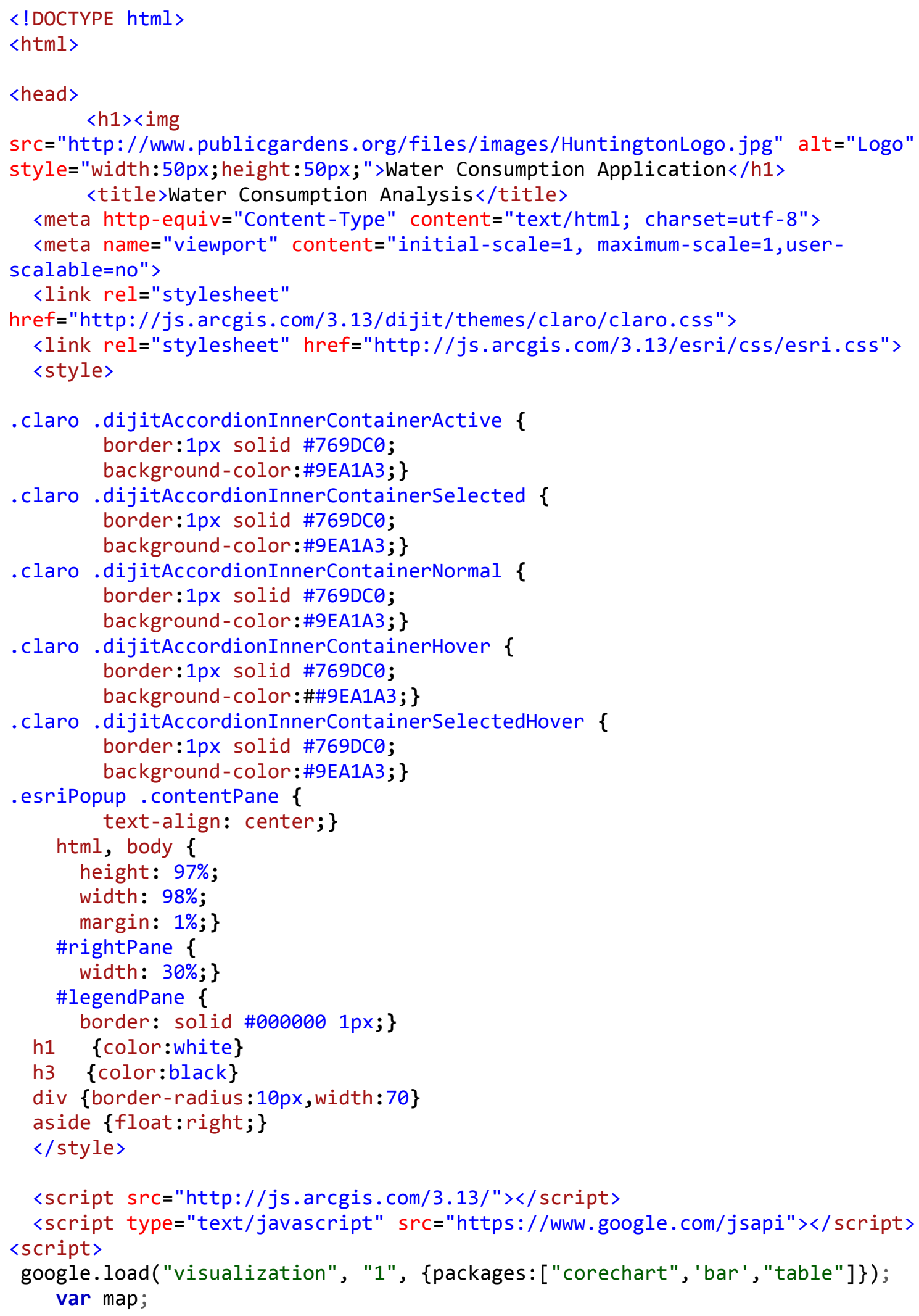




\section{require( [}

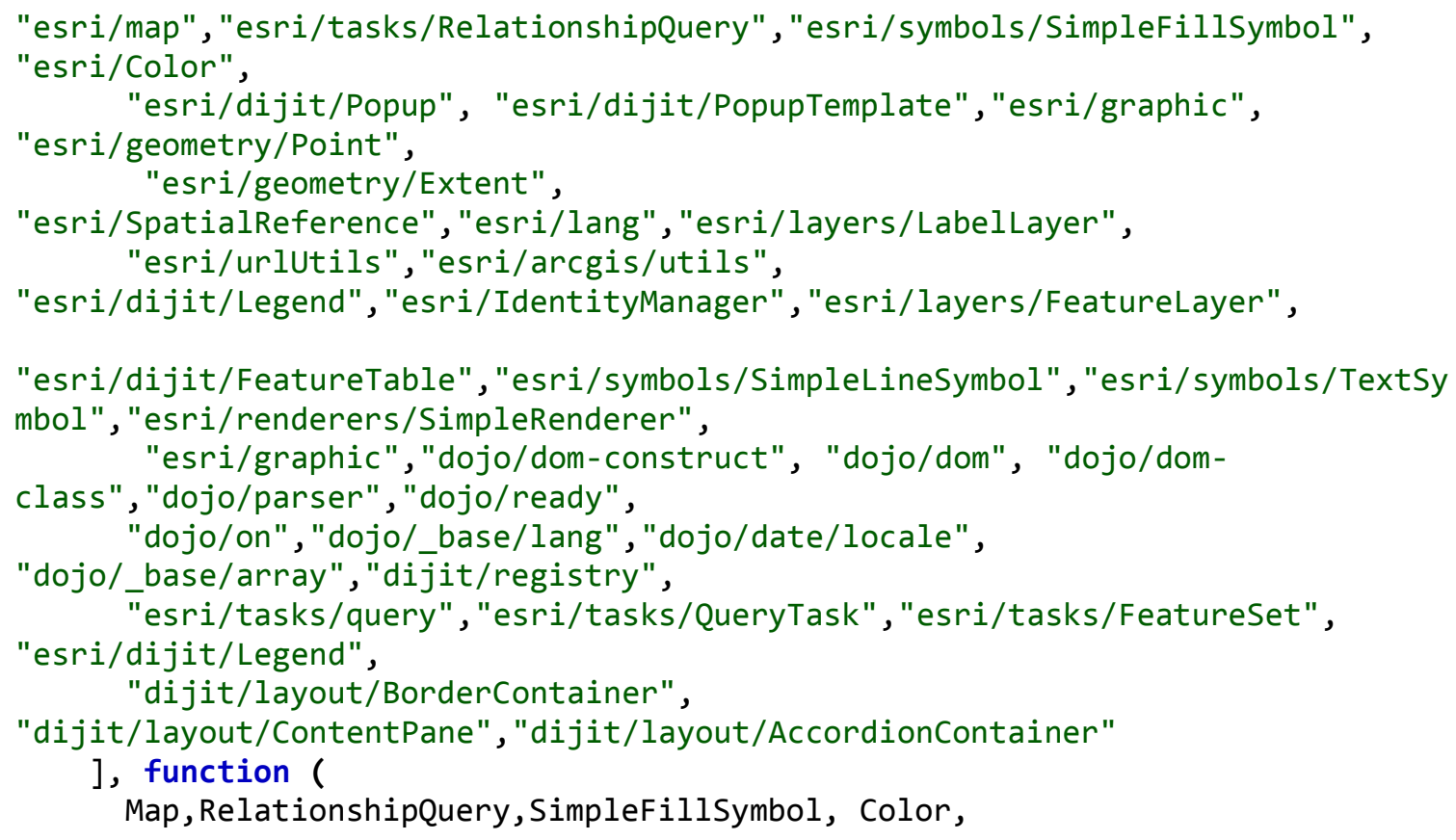

Popup, PopupTemplate, Graphic, Point, Extent, SpatialReference, esriLang, Label Layer, urlutils, arcgisUtils, Legend, esriID, FeatureLayer, FeatureTable,

SimpleLineSymbol, TextSymbol, SimpleRenderer, Graphic, domConstruct, dom, domclass, parser, ready, on, lang, locale, array, registry, Query, QueryTask, FeatureSet, Legend, BorderContainer, ContentPane

) \{

parser.parse();

buttonUpdate. disabled="disabled";

buttonUpdate2. disabled="disabled";

deleteButton2. disabled="disabled";

deleteButton. disabled="disabled";

//Edit URL's to Features Classes here

var

urlGardenBounds="http://services1.arcgis.com/ikHe2GBZOgUVQ79G/arcgis/rest/serv ices/Garden_Boundaries/FeatureServer/0";

var

urlGardenConsume="http: //services1. $\operatorname{arcgis.com/ikHe2GBZOgUVQ79G/arcgis/rest/ser~}$

vices/Garden_Boundaries/FeatureServer/1";

var

urlPipes="http://services1.arcgis.com/ikHe2GBZOgUVQ79G/ArcGIS/rest/services/Ir

rigation_Maintenance/FeatureServer/1";

var

urlValves="http://services1.arcgis.com/ikHe2GBZOgUVQ79G/arcgis/rest/services/I rrigation_Maintenance/FeatureServer/0";

var

urlMaintenanceTable="http: //services1. arcgis.com/ikHe2GBZOgUVQ79G/ArcGIS/rest/ services/Irrigation_Maintenance/FeatureServer/2";

var

urlReports="http://services1. arcgis.com/ikHe2GBZOgUVQ79G/arcgis/rest/services/ Irrigation_Notes/FeatureServer/0"; 







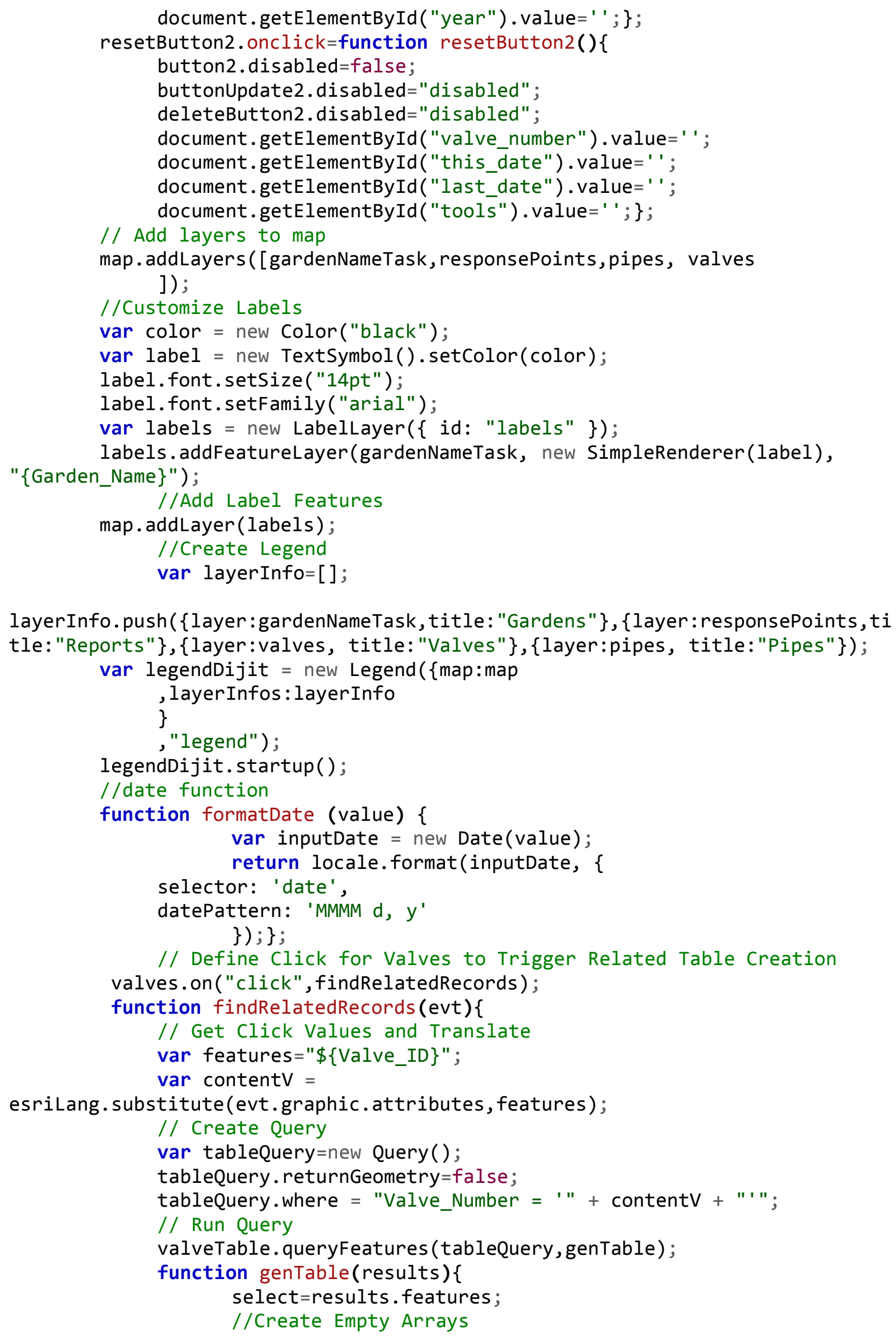

layerInfo.push ( $\{$ layer:gardenNameTask, title: "Gardens"\}, \{layer: responsePoints, ti tle:"Reports"\}, \{layer:valves, title:"Valves"\}, \{layer:pipes, title:"Pipes"\});

var legendDijit $=$ new Legend $(\{$ map:map , layerInfos: layerInfo 
$\mathrm{vId}=[]$;

vDate $=[]$;

vTool $=[]$

vobjectID $=[]$;

vTableAll=[];

//Cycle through values from Query, input into Arrays

for ( $i$ in select) \{

vId.push(select [i].attributes.Valve_Number);

VDate $=$ formatDate $($ select $[i]$. attributes.Date_Tested); vDate.push(VDate);

vTool.push (select[i].attributes.Tools);

vobjectID.push (select[i].attributes.OBJECTID);

vTableAll.push([vobjectID[i]

, vId[i]

, vDate[i]

, vTool[i]

]

\};

// Create Table

function makeTable()\{

var dataTable = new google.visualization.DataTable( );

dataTable.addColumn( 'number', ' ObjectID ');

dataTable.addColumn( 'string', ' ID');

dataTable.addColumn( 'string', 'date');

dataTable.addColumn('string', 'Tool');

dataTable.addRows (vTableAll)

var $\operatorname{vTab}=$ new

google.visualization. Table(document.getElementById('tableNode' )); google.visualization.events.addListener(vTab, 'select',

triggerSelect2);

vTab.draw(dataTable, \{showRowNumber:false\});

function triggerselect2()\{

selection=vTab.getSelection();

iDSelect=dataTable .getValue ( selection [0] . row, $\theta)$;

var oID=iDSelect//document.getElementById("object2").value;

var tableUpdateQuery=new Query();

tableUpdateQuery. returnGeometry=false;

tableUpdateQuery. where $=$ "OBJECTID $=$ " + oID + " ";

valveTable.queryFeatures (tableUpdateQuery, updateID);

function updateID(results) \{

var featuresID=results. features;

//Return Error if ID is not found in Table

if (typeof featuresID[0]==='undefined') \{

document.getElementById("message2"). innerHTML= "Object ID

does not exist"; \}

else\{

//Populate HTML

document.getElementById("valve_number"). value=featuresID[0] . attributes.V alve_Number; 
document.getElementById("this_date").value=formatDate(featuresID[0].attr ibutes.Date_Tested);

document.getElementById("last_date").value=formatDate(featuresID[0] .attr ibutes.Previous_Test);

document.getElementById("tools") . value=featuresID[0] . attributes. Tools;

ID;

document.getElementById("object2") . value=featuresID[0] . attributes.OBJECT

Found";

document.getElementById("message2"). innerHTML= "Object ID

button2.disabled="disabled"; buttonUpdate2. disabled=false;

\}$;\}$ deleteButton2. disabled=false;

\}$;\}$

makeTable( );

\} ;

\} ;

//create the Queries

var gardenQuery=new Query();

gardenQuery . returnGeometry=false;

gardenQuery. where="1=1";

var gardenNamesQuery= new Query();

gardenNamesQuery . returnGeometry=false;

gardenNamesQuery . where = "1=1";

//execute queries

gardenNameTask.queryFeatures (gardenNamesQuery, resultsList);

function resultsList(results) \{

var resultCount=results. features. length;

list=results. features;

//Create Empty Arrays

names $=[]$;

ids $=[]$;

// Method of using Fragments to dynamically fill HTML Dropdowns

based on discussion

// at: http://stackoverflow.com/questions/11255219/use-a-

javascript-array-to-fill-up-a-drop-down-select-box

//Create Fragments from HTML select Elements

var gardenName=document.getElementById( 'idG' );

var gardenNameT=document.getElementById ( 'idGT');

fragId=document . createDocumentFragment ( );

fragIdT=document . createDocumentFragment ( );

//Iterate through list of Garden Names to fill Options HTML

for ( $i$ in list) \{

names.push(list[i].attributes.Garden_Name);

ids.push(1ist [i].attributes.Garden_ID); 


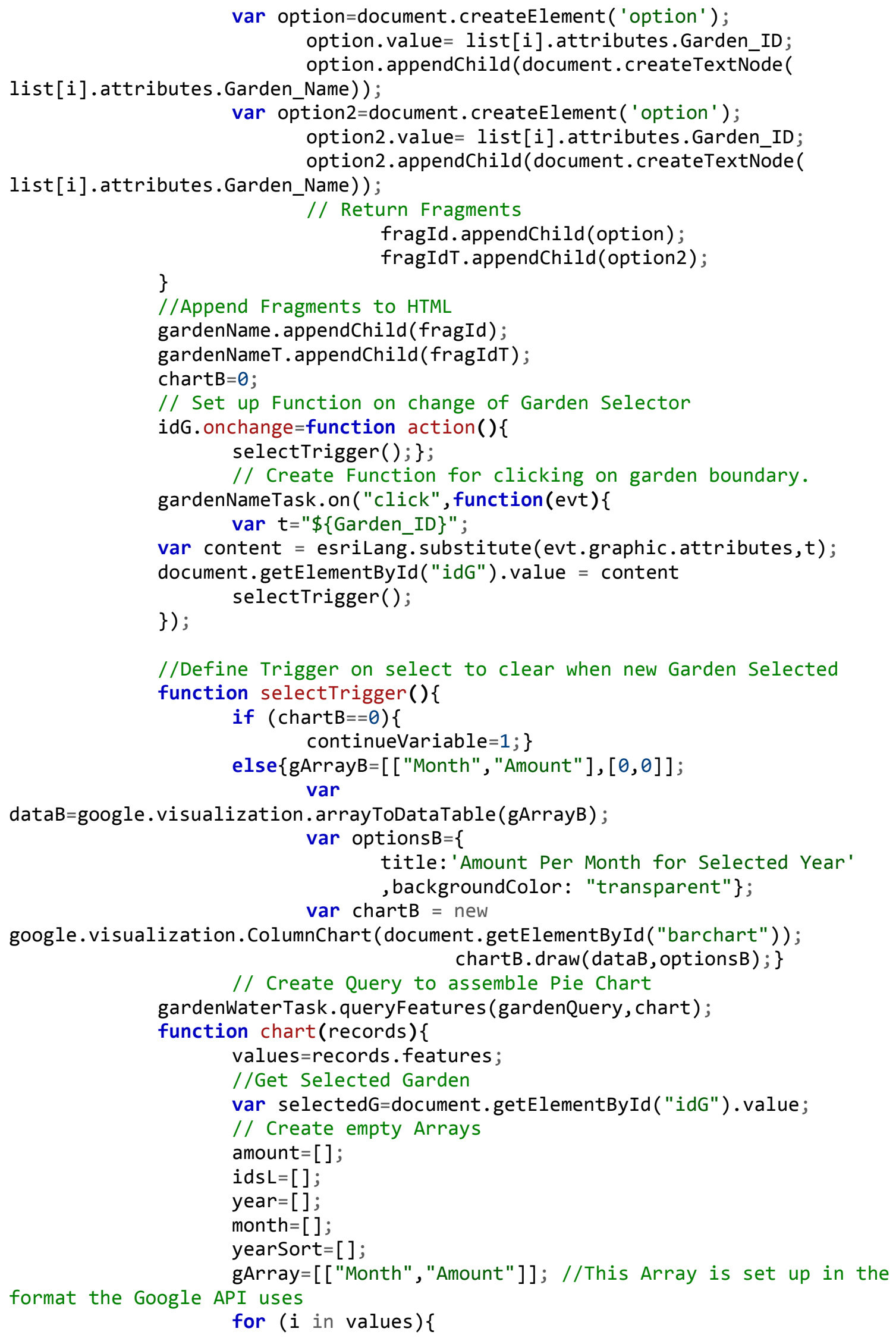


var nameCur=values [i] . attributes.Garden_Id;

if (nameCur $==$ selectedG) \{

// Push values to array if the garden name

equals the selected garden

amount.push (values [i] .attributes.Amount);

idsL.push (values [i] .attributes.Garden_Id);

month.push (values [i] . attributes.Month);

year.push (values [i].attributes.Year);

yearsort.push(values [i] . attributes.Year);

\}

else\{

continueVariable $=1 ;\}\}$;

//Iterate through years

yearV $=[$;

amountV $=[0]$;

yearSort.sort (function( $a, b)\{$ return $a-b\})$;

for ( $y$ in yearsort) \{

curTest=yearSort $[\mathrm{y}]$;

futTest=yearSort $[y-1]$;

if (curTest==futTest) \{

else \{

continueVariable $=1 ;\}$

for ( $i$ in yearV) \{ yearV.push(curTest); \}\}

for (y in year) \{

if $($ year $[y]==\operatorname{yearV}[i])\{$

if (amountV $[i]>=0)\{$

amountV $[\mathrm{i}]=$ amountV $[\mathrm{i}]+\operatorname{amount}[\mathrm{y}] ;\}$

else \{

amountV $[\mathrm{i}]=0$;

amountV $[\mathrm{i}]=$ amountV $[\mathrm{i}]+\operatorname{amount}[\mathrm{y}] ;\}\}$

else \{

for ( $i$ in yearV) \{

continueVariable $=1 ;\}\}\}$

workArray $=[$ yearV [i].tostring ( ), amountV[i]];

gArray.push (workArray);

//Draw Google Chart

function drawChart()\{

var chart $=0$;

var data=google.visualization . arrayToDataTable(

gArray

) ;

var options $=\{$

title:'Chart Comparing Consumption of Water

Per Year for Selected Garden'

, height: 400

, width: 400

\};

, backgroundColor: "transparent"

var chart $=$ new

google.visualization. LineChart (document.getElementById('linechart' ));

chart.draw(data, options); 


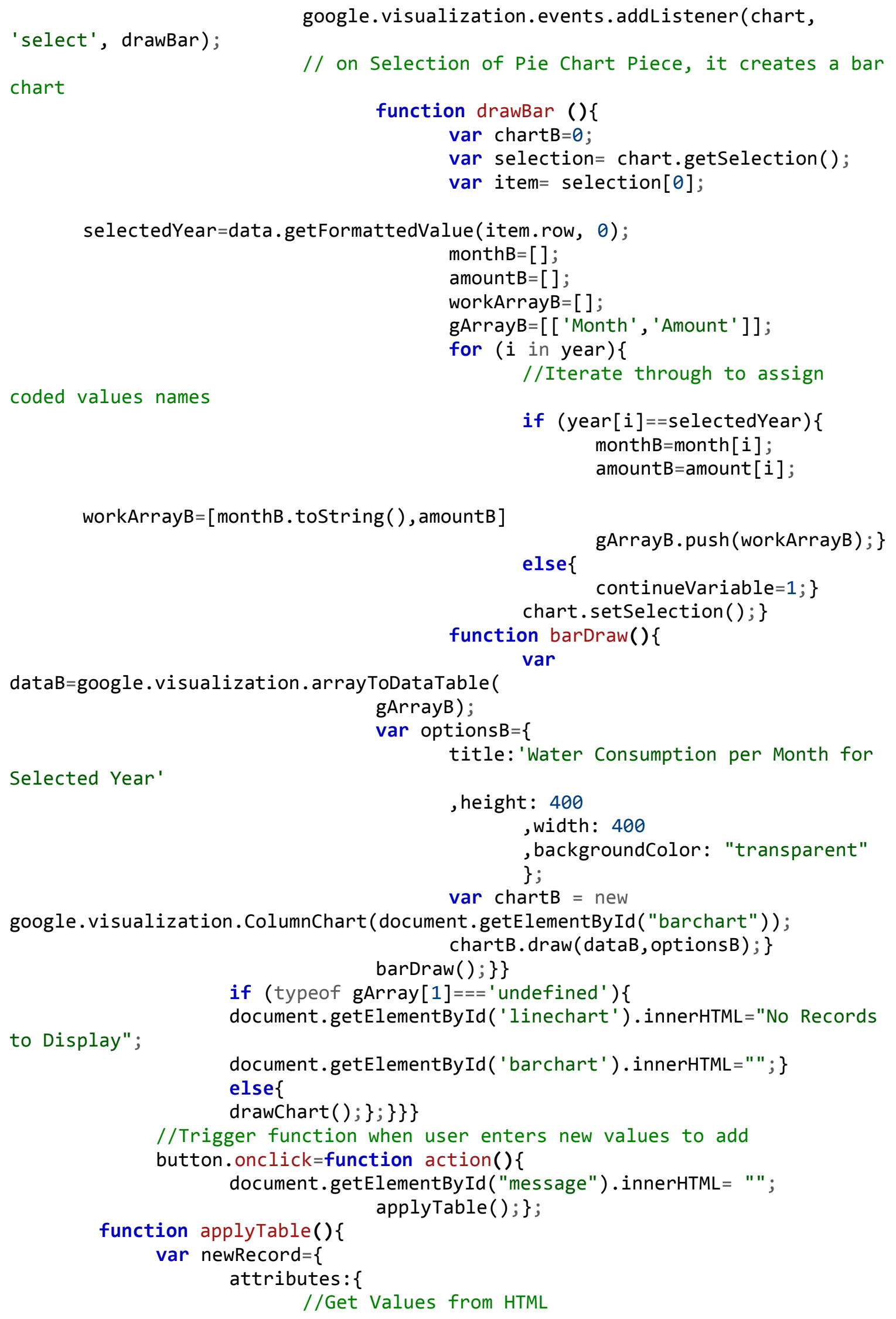




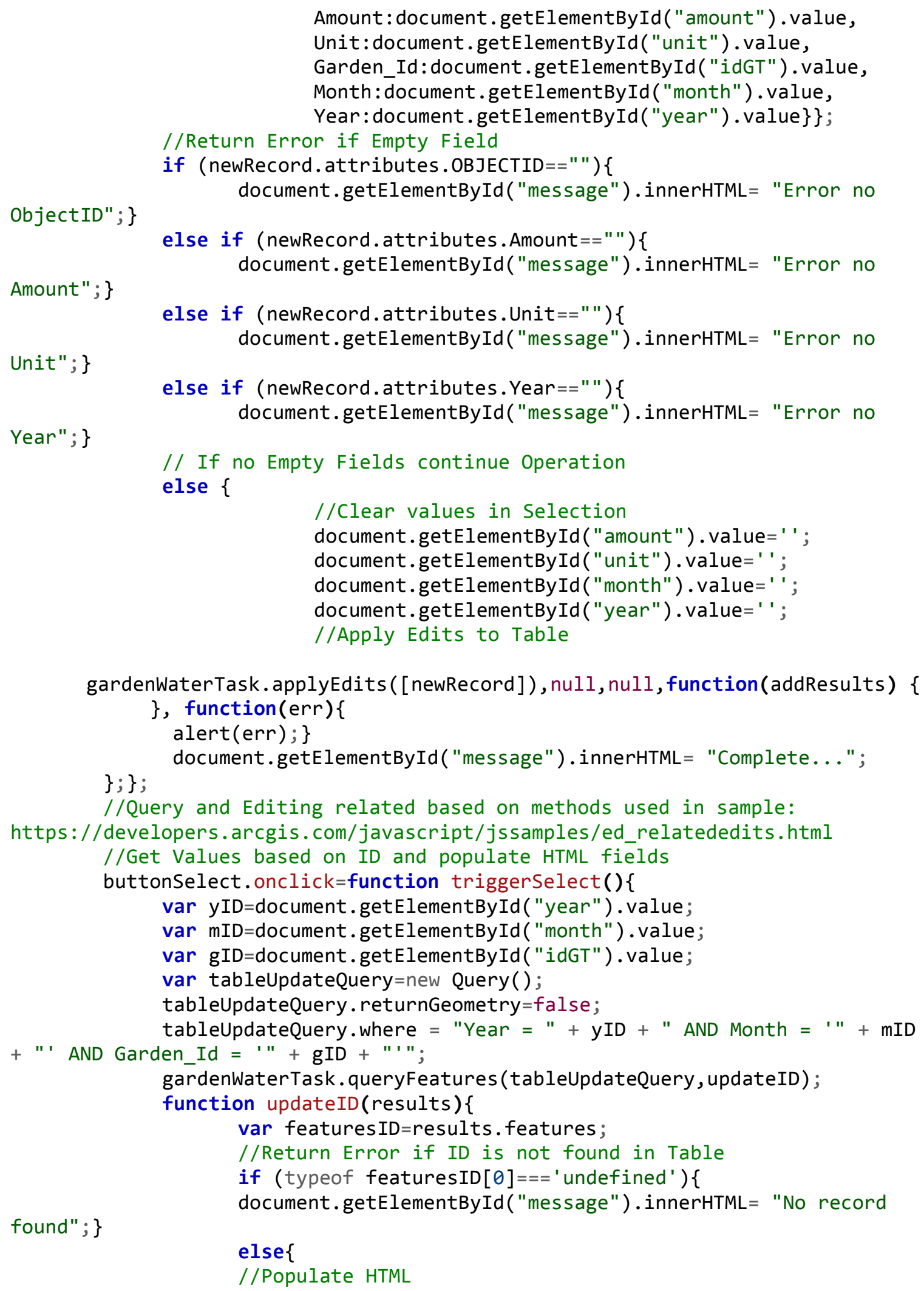


document.getElementById ("unit") . value=featuresID[0] . attributes. Unit;

document . getElementById ("month") . value=featuresID[0] . attributes. Month;

document.getElementById ("year") . value=featuresID [0] . attributes.Year;

document .getElementById("idGT").value=featuresID[0] . attributes.Garden_Id

;

document.getElementById("object") .value=featuresID[0] . attributes. OBJECTI

D;

Found";

document.getElementById("message").innerHTML= "Object ID

button. disabled="disabled";

buttonUpdate. disabled=false;

deleteButton. disabled=false;

\}$;\}\}$;

//Populate HTML Fields based on Search

buttonSelectID. onclick=function triggerSelect2()\{

var oID=document. getElementById("object"). value;

var tableUpdateQuery=new Query();

tableUpdateQuery. returnGeometry=false;

tableUpdateQuery. where $=$ "OBJECTID $="$ + oID + " ";

gardenWaterTask.queryFeatures (tableUpdateQuery, updateID);

function updateID(results) \{

var featuresID=results. features;

//Return Error if ID is not found in Table

if (typeof featuresID[0]==='undefined ') \{

does not exist"; \}

document.getElementById("message"). innerHTML= "Object ID

else\{

//Populate HTML

document.getElementById ("amount") .value=featuresID[0] . attributes . Amount;

document.getElementById("unit") . value=featuresID[0]. attributes. Unit;

document . getElementById ("month") . value=featuresID[0] . attributes. Month;

document.getElementById ("year").value=featuresID[0] . attributes.Year;

document.getElementById ("idGT").value=featuresID[0] . attributes.Garden_Id

;

Found";

document.getElementById("message").innerHTML= "Object ID

button. disabled="disabled";

buttonUpdate . disabled=false;

deleteButton . disabled=false;

\};\} \};

//Functionality for Delete Button to Remove Records from

Consumption Logs

deleteButton. onclick=function deletelog ()\{

var newRecord $=\{$

attributes: \{ 
OBJECTID: document.getElementById("object") . value, Amount: document.getElementById("amount").value, Unit: document.getElementById("unit").value, Garden_Id:document.getElementById("idGT"). value, Month: document.getElementById("month"). value, \} Year: document.getElementById("year").value\}

//Clear HTML Forms

document.getElementById("object2") . value= ' ' ; document.getElementById ("object") value=" ' ; document.getElementById ("amount") . value= " '; document.getElementById("unit") .value=" ' ; document.getElementById("month") . value= ' '; document.getElementById("year") . value= " ' ; gardenWaterTask.applyEdits (null, null, [newRecord]); document . getElementById ("message $2 ")$. innerHTML=

"Complete...";

\};

button. disabled=false;

buttonUpdate. disabled="disabled";

deleteButton.disabled="disabled";

//Function to Apply Changes to fields to Object with ID

buttonUpdate. onclick=function updateTable()\{

var newRecord $=\{$

attributes: \{

OBJECTID: document .getElementById("object").value, Amount: document.getElementById("amount").value, Unit: document.getElementById("unit").value, Garden_Id:document.getElementById("idGT"). value, Month: document.getElementById("month").value, Year: document.getElementById("year"). value \}\};

//Return Error if Empty Field

if (newRecord.attributes.OBJECTID $=="$ ") \{

document.getElementById("message" $)$. innerHTML= "Error no

ObjectID";

\}

else if (newRecord.attributes. Amount $=="$ ") \{ document.getElementById("message"). innerHTML= "Error no

Amount";

\}

else if (newRecord.attributes.Unit $=="$ ") \{

document.getElementById("message"). innerHTML= "Error no

Unit";

\}

else if (newRecord.attributes.Year==" ") \{

document.getElementById("message"). innerHTML= "Error no

Year";

\}

// If no Empty Fields continue Operation

else \{

document.getElementById ("object") value= ' ' ; document.getElementById ("amount") .value=" '; document.getElementById ("unit") . value=" ' ; document.getElementById ("month") . value=" ' ; 


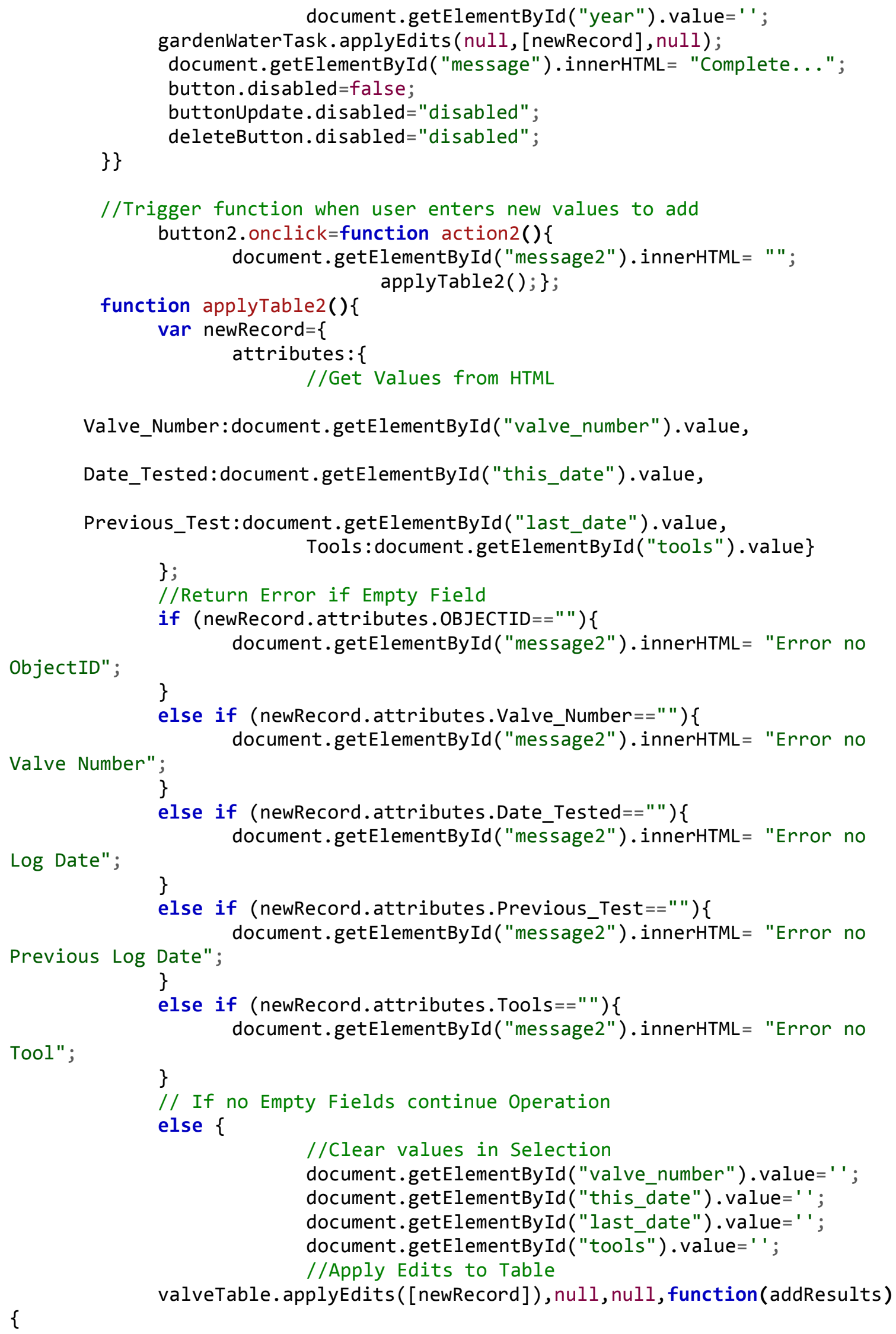




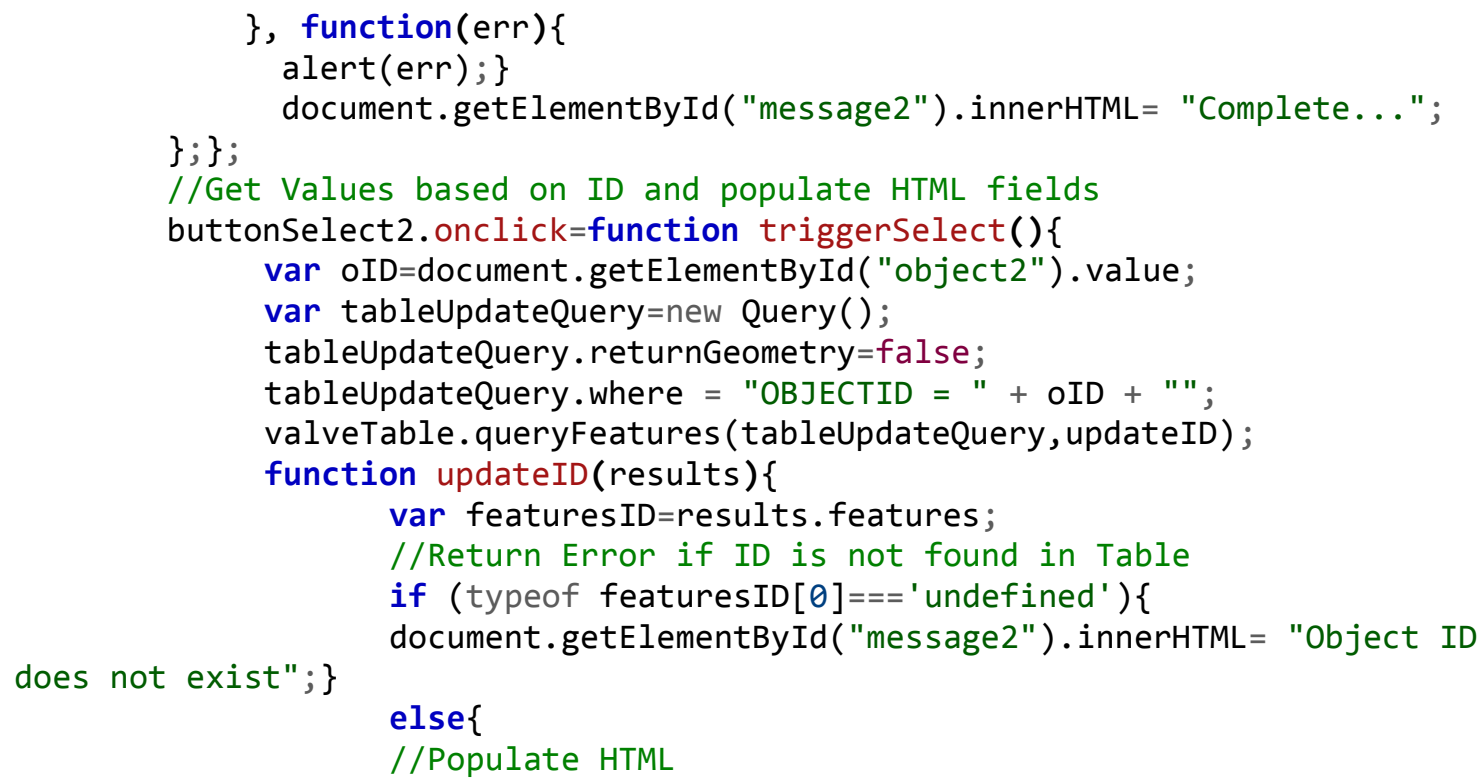

document.getElementById("valve_number").value=featuresID[0] . attributes.V alve_Number;

document.getElementById ("this_date") . value=formatDate (featuresID[ $\theta$ ]. attr ibutes.Date_Tested);

document.getElementById("last_date") . value=formatDate (featuresID[ $\theta$ ]. attr ibutes.Previous_Test);

document.getElementById ("tools").value=featuresID[0].attributes.Tools; document.getElementById("message2"). innerHTML= "Object ID

Found";

//Enable and Disable Buttons button2.disabled="disabled"; buttonUpdate2. disabled=false; deleteButton2. disabled=false;

\}$;\}\} ;$

//Function to Delete Valve Maintenance Log when delete button is

pressed

deleteButton 2 . onclick=function deletevalve( $)\{$

var newRecord $=\{$

attributes: \{

OBJECTID: document .getElementById("object2").value,

Valve_Number:document.getElementById("valve_number").value,

Date_Tested:document.getElementById("this_date").value,

Previous_Test:document.getElementById("last_date").value,

\};

Tools: document.getElementById("tools").value\}

document .getElementById("object2") . value= " ';

document.getElementById("valve_number"). value=" ' ;

document.getElementById("this_date").value=" ' ;

document.getElementById("last_date").value=" '; 


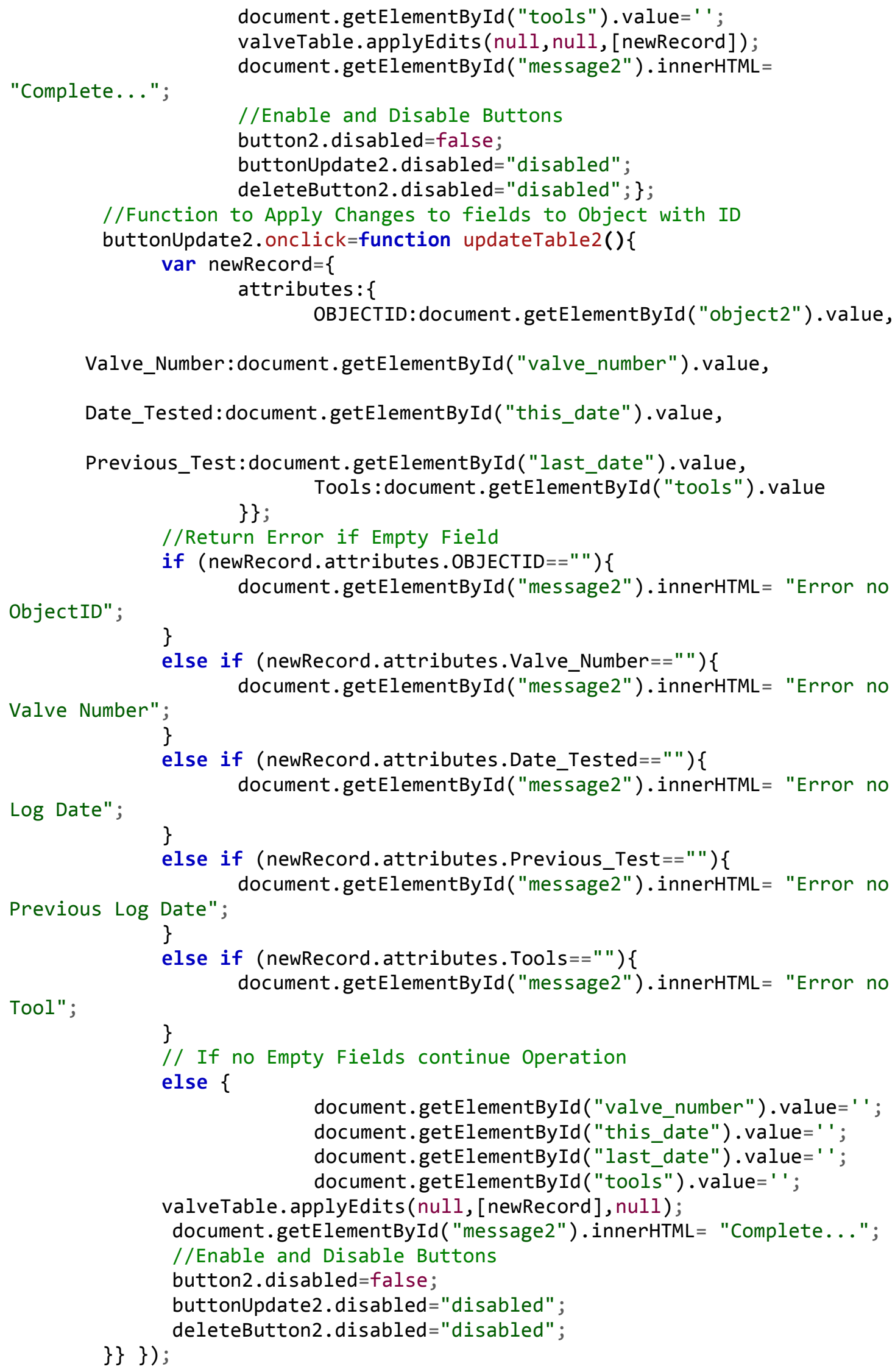




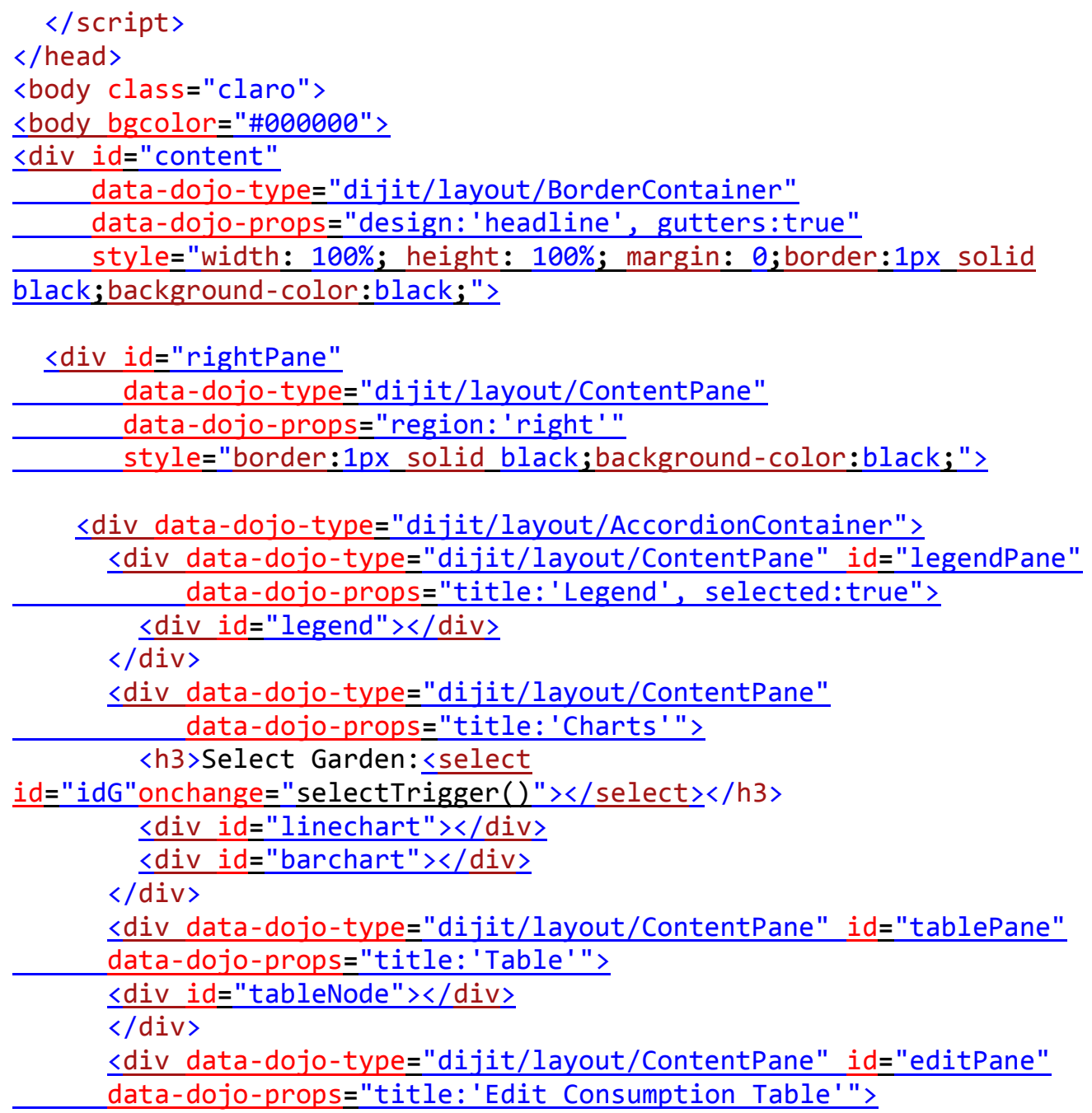

Garden: $\langle b r\rangle\langle$ select id="idGT" $\rangle\langle/$ select $\rangle\langle b r\rangle$

Month: $\langle$ br $\rangle\langle$ select id="month"> <option value="Jan">January</option> 


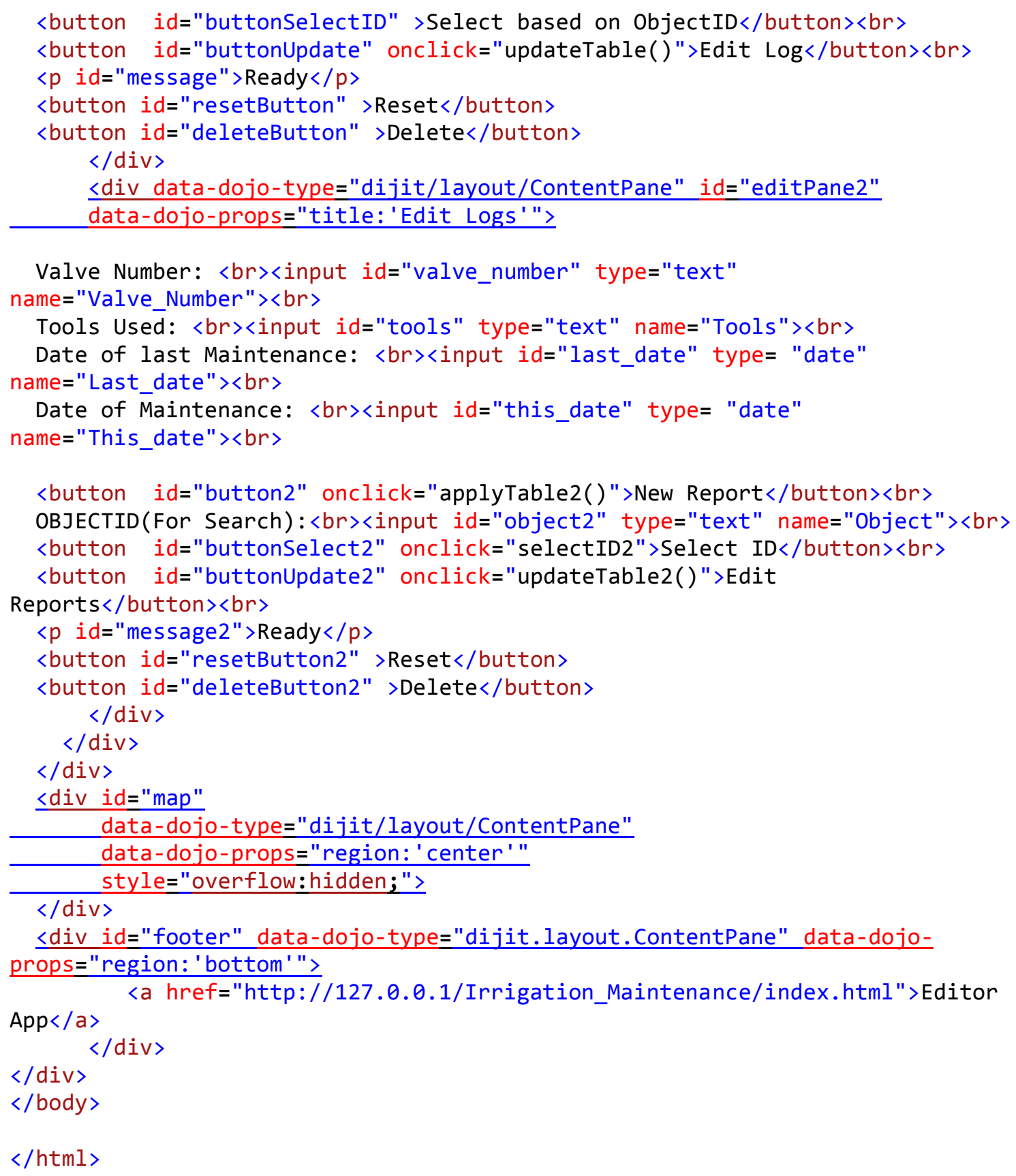




\section{Appendix B. Code for Report Application}

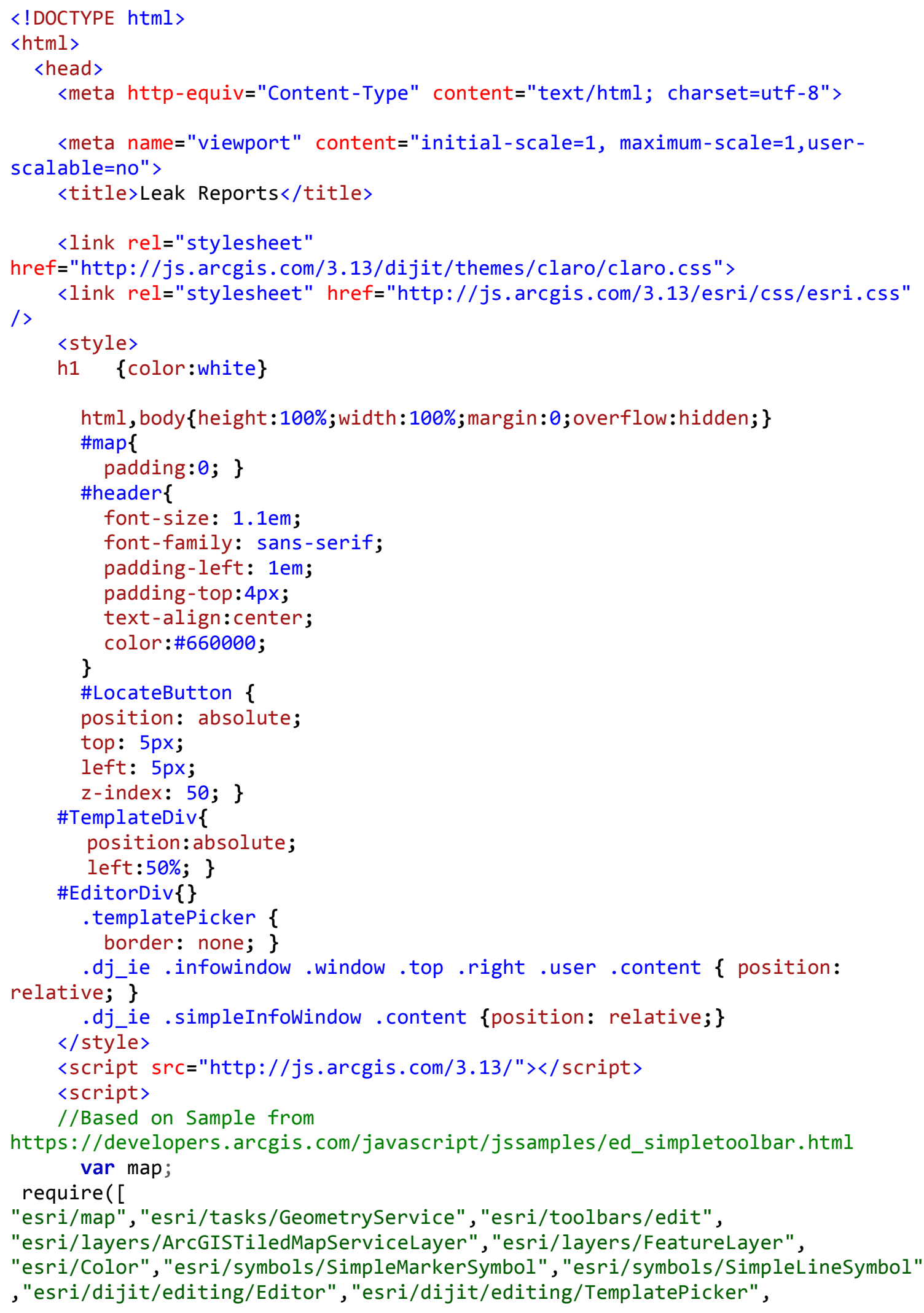




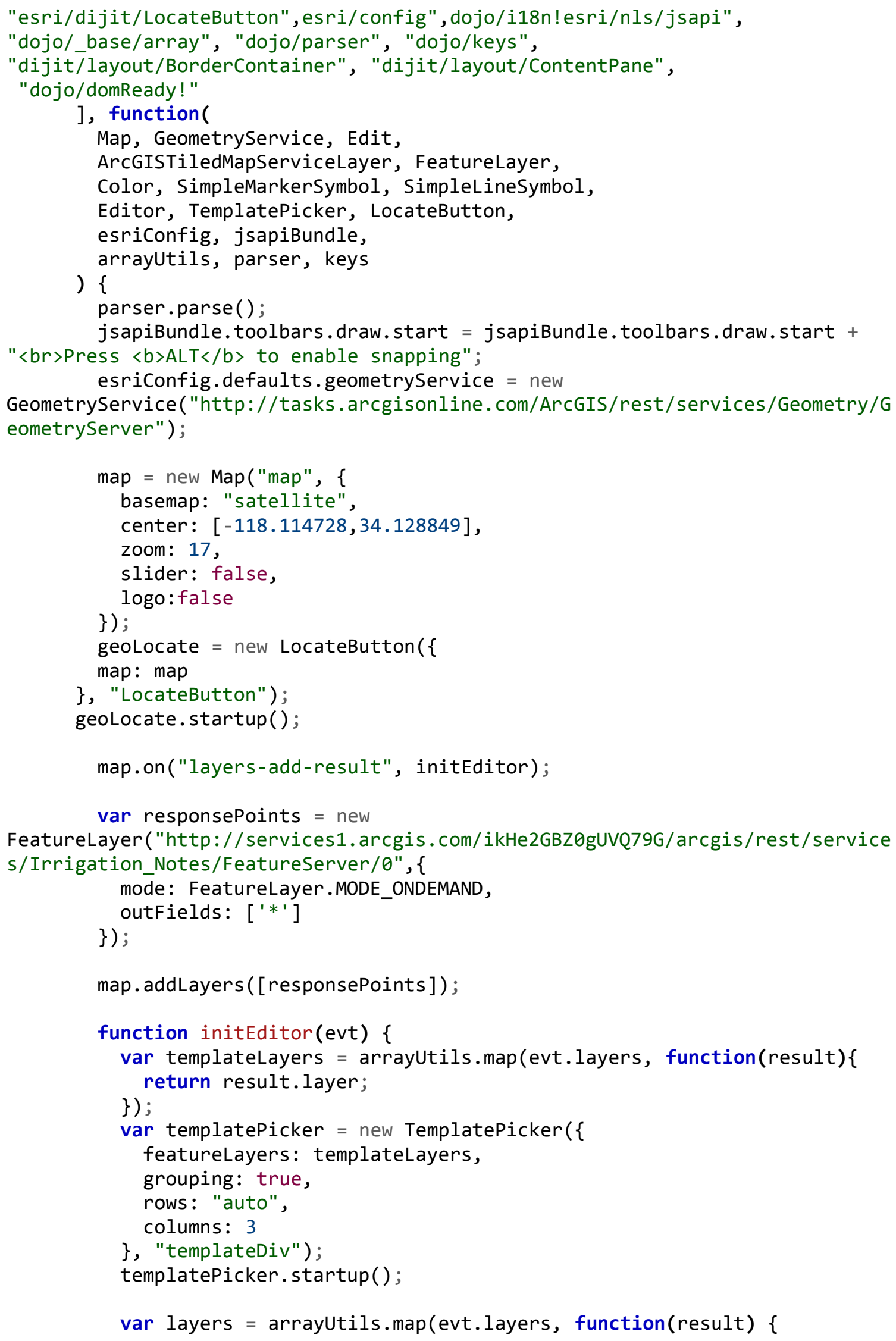


\});

return $\{$ featureLayer: result.layer \};

var settings $=\{$

map: map,

templatePicker: templatePicker,

layerInfos: layers, toolbarVisible: true, createOptions: \{

polylineDrawTools:[ Editor.CREATE_TOOL_FREEHAND_POLYLINE ], polygonDrawTools: [ Editor.CREATE_TOOL_FREEHAND_POLYGON, Editor. CREATE TOOL CIRCLE, Editor.CREATE_TOOL_TRIANGLE, ] Editor.CREATE_TOOL_RECTANGLE

\} ,

toolbarOptions: \{

\}

reshapeVisible: true

\} ;

var params $=$ \{settings: settings $\}$;

var myEditor = new Editor (params, 'editorDiv');

//define snapping options

var symbol = new SimpleMarkerSymbol (

SimpleMarkerSymbol.STYLE_CIRCLE, 15 ,

new SimpleLineSymbol(

SimpleLineSymbol.STYLE_SOLID, new $\operatorname{Color}([0,255,0,0.5])$,

5

) ,

null

) ;

\}$)$;

myEditor.startup ( );\}

$\langle/$ script $\rangle$

$\langle/$ head $\rangle$

$\langle$ body class="claro" >

$\langle$ body bgcolor $=" \# 000000 "\rangle$

<div id="main" data-dojo-type="dijit/layout/BorderContainer" data-dojoprops="design: 'headline'" style="height:width:100\%; height:100\%;">

<div data-dojo-type="dijit/layout/ContentPane" id="header" data-dojoprops="region: 'top'" style="width:10\%; height:10\%; overflow:hidden; background color:\#000000" $>$

$<$ h1><img

src="http://www.publicgardens.org/files/images/HuntingtonLogo.jpg" alt="Logo" style="width:30px; height:30px" $>\langle$ font size="5" $>$ \&emsp; Irrigation Report

App $</$ font $>\langle/$ h1 $>$

$\langle/$ div $\rangle$

<div data-dojo-type="dijit/layout/ContentPane" data-dojo-

props="region: 'bottom'" style="height: 120px;width:100px;overflow:hidden;"> $\langle$ div id="templateDiv" $\rangle\langle/$ div $\rangle$

$\langle$ div id="editorDiv" $\rangle\langle/$ div $\rangle$ 


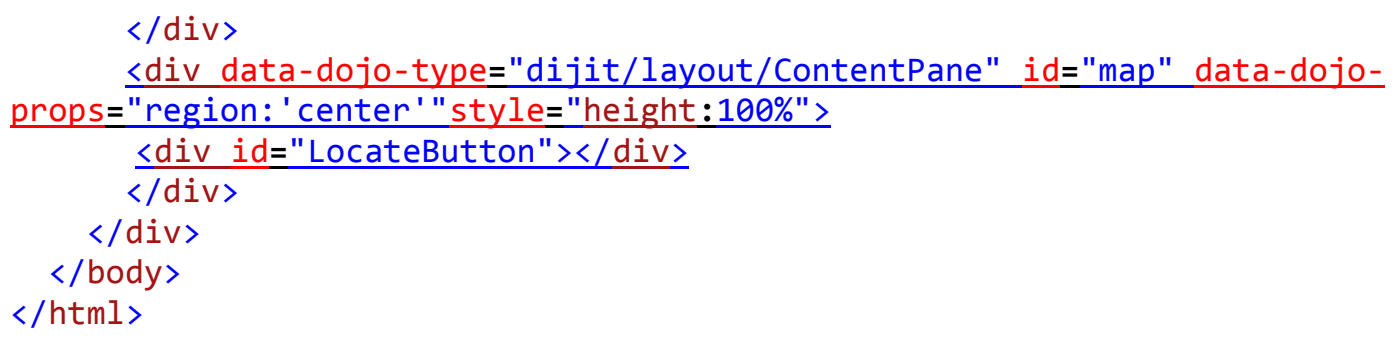




\section{Appendix C. Valve ID Assignment Script}

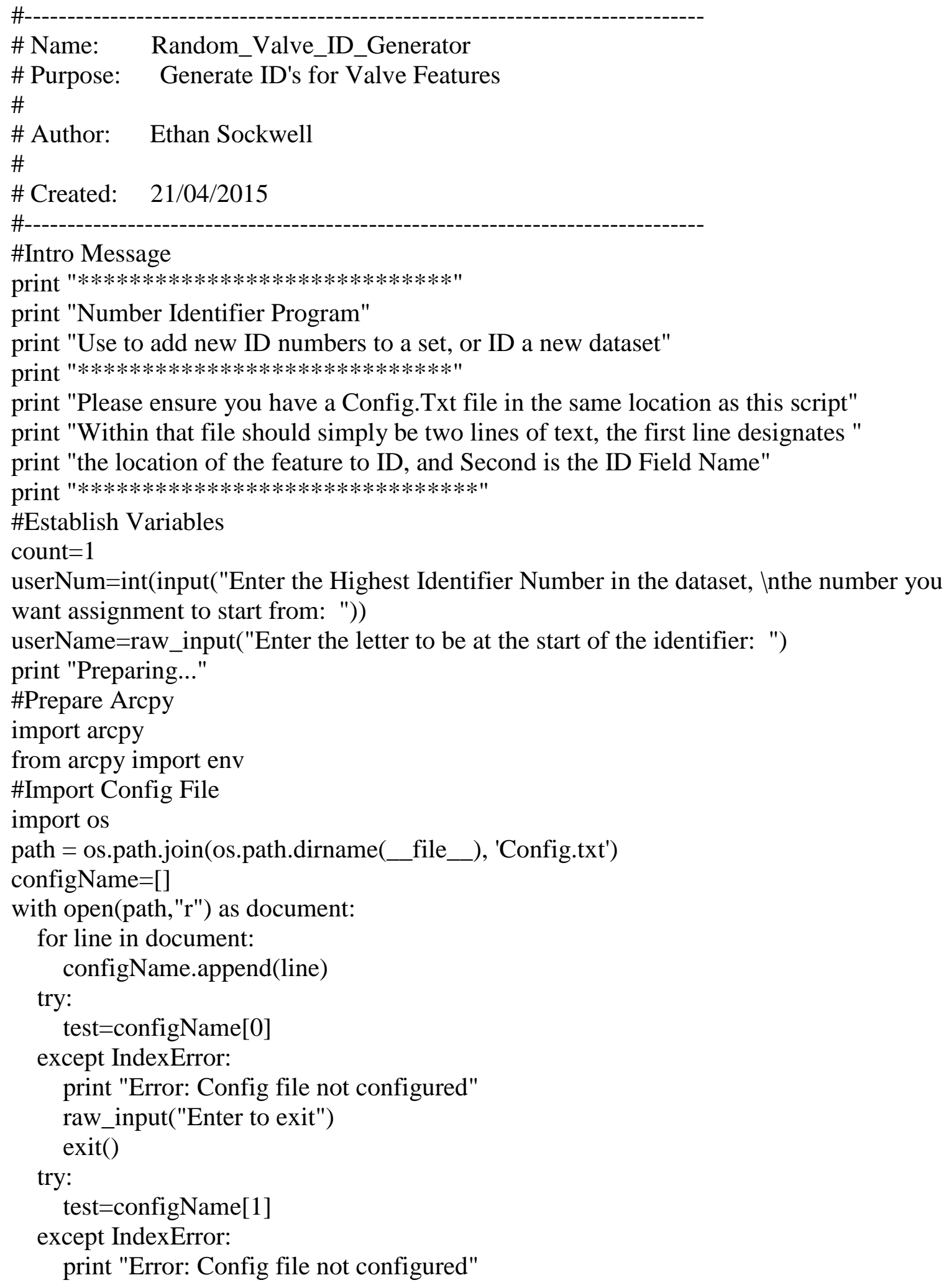




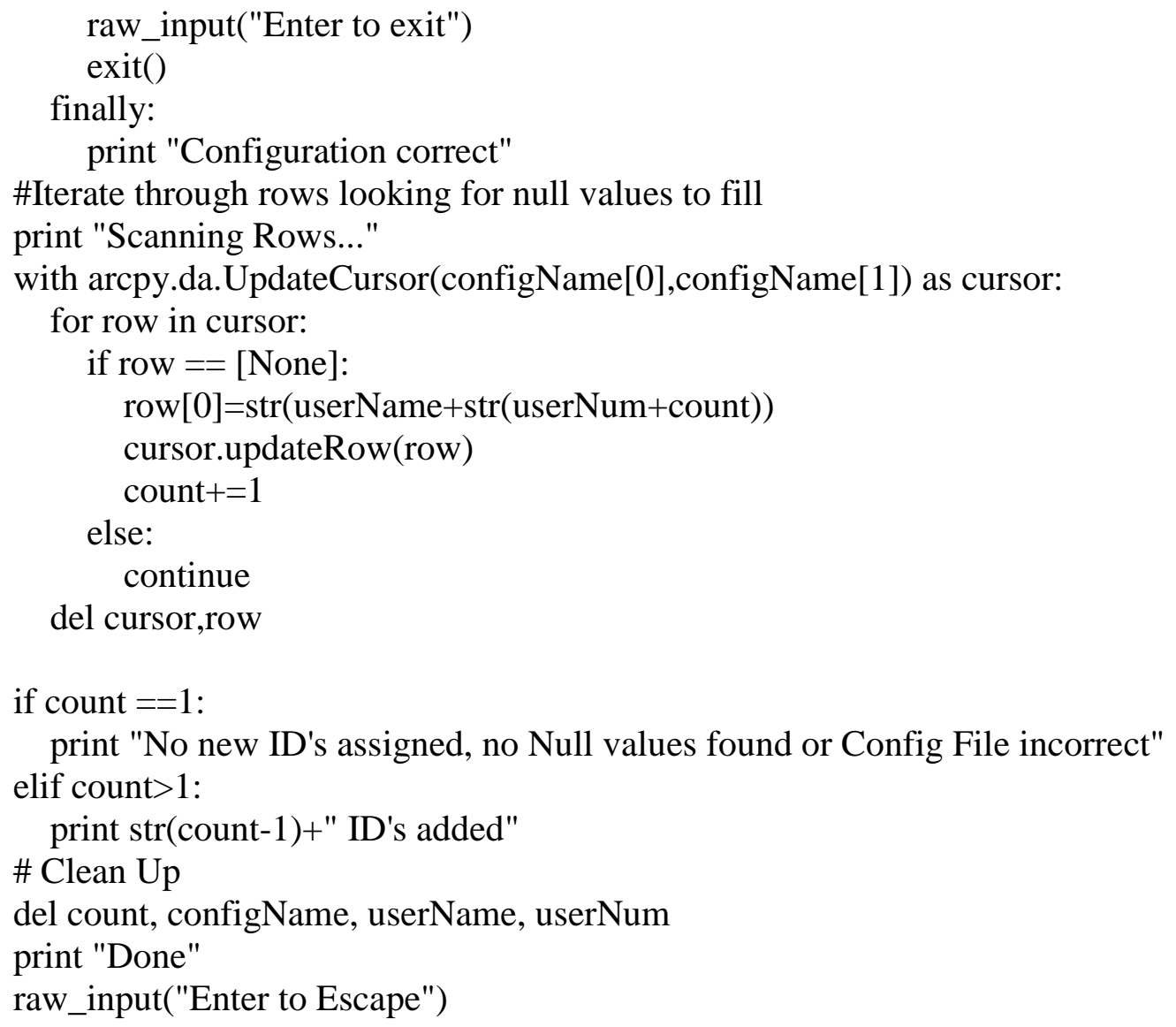




\section{Appendix D. Management Application File Structure}

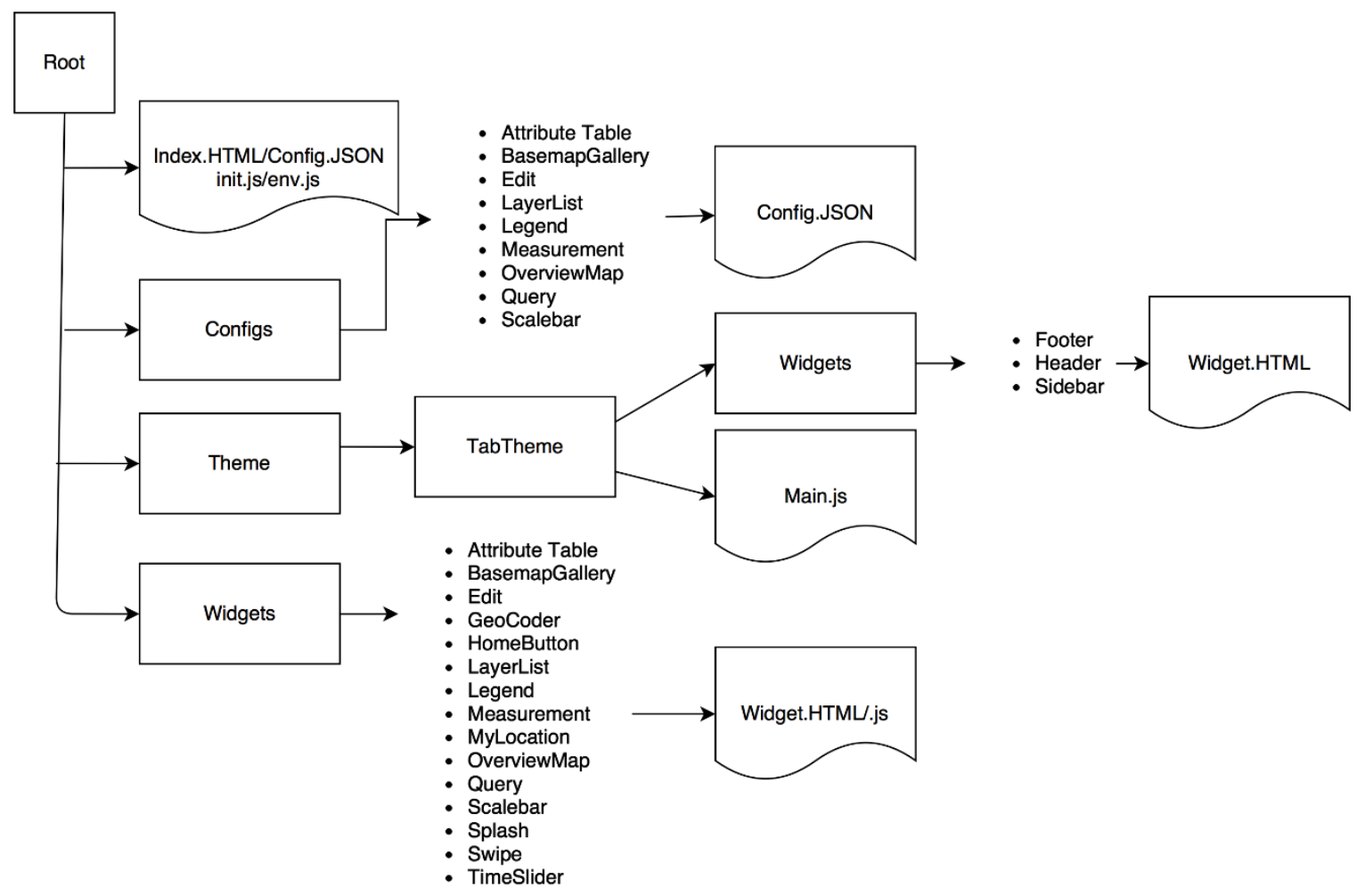

